University of Massachusetts Amherst

ScholarWorks@UMass Amherst

Open Access Dissertations

$2-2010$

\title{
An Assessment of The Nonparametric Approach for Evaluating The Fit of Item Response Models
}

Tie Liang

University of Massachusetts Amherst

Follow this and additional works at: https://scholarworks.umass.edu/open_access_dissertations

Part of the Education Commons

\section{Recommended Citation}

Liang, Tie, "An Assessment of The Nonparametric Approach for Evaluating The Fit of Item Response Models" (2010). Open Access Dissertations. 176.

https://doi.org/10.7275/1264891 https://scholarworks.umass.edu/open_access_dissertations/176

This Open Access Dissertation is brought to you for free and open access by ScholarWorks@UMass Amherst. It has been accepted for inclusion in Open Access Dissertations by an authorized administrator of ScholarWorks@UMass Amherst. For more information, please contact scholarworks@library.umass.edu. 
AN ASSESSMENT OF THE NONPARAMETRIC APPROACH

FOR EVALUATING THE FIT OF ITEM RESPONSE MODELS

A Dissertation Presented

by

TIE LIANG

Submitted to the Graduate School of the

University of Massachusetts Amherst in partial fulfillment

of the requirements for the degree of

DOCTOR OF EDUCATION

February 2010

School of Education 
(C) Copyright by Tie Liang 2010

All Rights Reserved 
AN ASSESSMENT OF THE NONPARAMETRIC APPROACH

FOR EVALUATING THE FIT OF ITEM RESPONSE MODELS

A Dissertation Presented

by

TIE LIANG

Approved as to style and content by:

Craig S. Wells, Chair

Ronald K. Hambleton, Member

Anna Liu, Member

Christine B. McCormick, Dean

School of Education 


\section{ACKNOWLEDGEMENTS}

First of all, I would like to thank my committee members: Craig Wells, Ron Hambleton, and Anna Liu. I have benefited a lot from their broad knowledge and great insights. Their supports and patience encouraged me to finish this dissertation. Craig, as my advisor, has been teaching and supporting me with his wisdom and kind criticism throughout my graduate studies. He is such an easy-going and nice person that I feel he is not only my professor but also my good friend. I would also like to thank Ron for his tremendous help on both my academic study and career choice. I learned a lot from his seriousness in dealing with problems and gained a lot from his inspiration on my deeper thinking. In addition, I was impressed by his great personality. One sentence he ever told me and will be embedded in my heart is that "being generous can bring you a lot of credits”. Although Anna and I did not have any close interaction before, I was so lucky to have her agree to be on my committee. Through my contact and talk with her during my dissertation process, I found all my friends' evaluation on her was correct: she is a very smart, experienced and outstanding statistician.

I am also grateful to other people who have helped me during my four years study at REMP. I will never forget Steve's exceptional guidance on my assignments and projects. His care and understanding to students touched me a lot. Lisa's humor and teaching skill helped me learn statistics in a special way. All my fellow students deserve my hug for their friendship.

Finally, but most importantly, I would like to thank my husband and daughter for their love, which is a unique gift I already got and will always cherish. I would also like to thank my dear parents and my aunt who passed away two years ago. What I have been learning from them becomes invaluable treasures in my whole life. The 
achievement of each assignment and project during my graduate study and the completion of the current dissertation came from what they told me: "no pain, no gain." 


\begin{abstract}
AN ASSESSMENT OF THE NONPARAMETRIC APPROACH

FOR EVALUATING THE FIT OF ITEM RESPONSE MODELS
\end{abstract}

FEBRUARY, 2010

TIE LIANG, B.S., CAPITAL UNIVERSITY OF ECONOMICS AND BUSINESS

M.S., UNIVERSITY OF MASSACHUSETTS AMHERST

Ed.D., UNIVERSITY OF MASSACHUSETTS AMHERST

Directed by: Professor Craig S. Wells

As item response theory (IRT) has developed and is widely applied, investigating the fit of a parametric model becomes an important part of the measurement process when implementing IRT. The usefulness and successes of IRT applications rely heavily on the extent to which the model reflects the data, so it is necessary to evaluate model-data fit by gathering sufficient evidence before any model application. There is a lack of promising solutions on the detection of model misfit in IRT. In addition, commonly used fit statistics are not satisfactory in that they often do not possess desirable statistical properties and lack a means of examining the magnitude of misfit (e.g., via graphical inspections).

In this dissertation, a newly-proposed nonparametric approach, RISE was thoroughly and comprehensively studied. Specifically, the purposes of this study are to (a) examine the promising fit procedure, RISE, (b) compare the statistical properties of $R I S E$ with that of the commonly used goodness-of-fit procedures, and (c) investigate how RISE may be used to examine the consequences of model misfit.

To reach the above-mentioned goals, both a simulation study and empirical study were conducted. In the simulation study, four factors including ability distribution, sample size, test length and model were varied as the factors which may influence the 
performance of a fit statistic. The results demonstrated that RISE outperformed $G^{2}$ and $S-X^{2}$ in that it controlled Type I error rates and provided adequate power under all conditions. In the empirical study, the three fit statistics were applied to one empirical data and the misfitting items were flagged. RISE and $S-X^{2}$ detected reasonable numbers of misfitting items while $G^{2}$ detected almost all items when sample size is large. To further demonstrate an advantage of RISE, the residual plot on each misfitting item was shown. Compared to $G^{2}$ and $S-X^{2}, R I S E$ gave a much clearer picture of the location and magnitude of misfit for each misfitting item.

Other than statistical properties and graphical displays, the score distribution and test characteristic curve (TCC) were investigated as model misfit consequence. The results indicated that for the given data, there was no practical consequence on classification before and after replacement of misfitting items detected by three fit statistics. 


\section{CONTENTS}

Page

ACKNOWLEDGEMENTS.....................................................

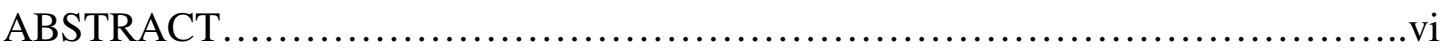

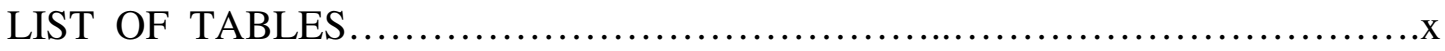

LIST OF FIGURES .......................................................

\section{CHAPTER}

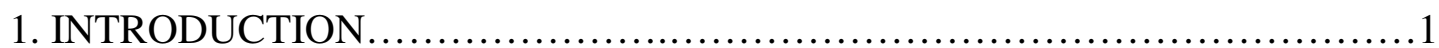

1.1 Background.......................................................

1.2 Statement of the Problem...........................................

1.3 Objectives of the Study............................................. 4

1.4 Outline of the Study...................................................

2. REVIEW OF LITERATURE................................................

2.1 IRT Assumptions and Models..........................................5

2.2 Model Data Fit Procedures.............................................. 7

2.3 Empirical Research on Fit Indices..................................14

2.4 Summary.................................................... 21

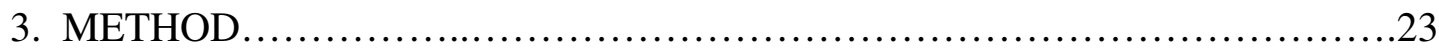

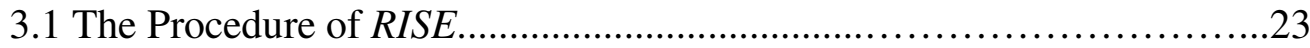

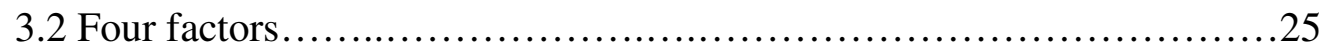

3.3 Data Generation................................................. 27

3.4 Data Analyses..................................................... 28

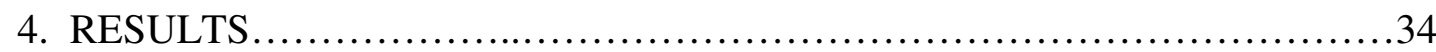

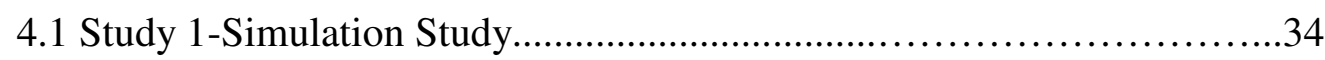

4.1.1 Statistical Properties.......................................34

4.1.2 Model Misfit Consequence.....................................38

4.1.2.1 Score Distribution...................................... 38

4.1.2.2 TCC ................................................

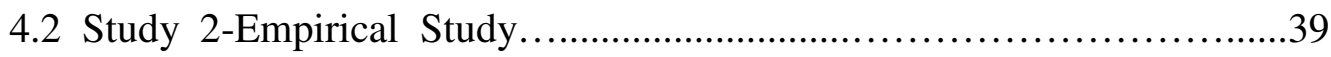

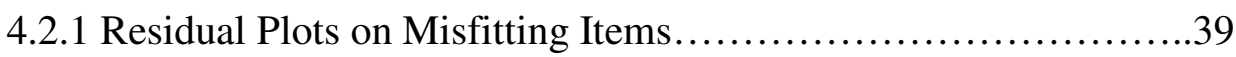

4.2.2 Model Misfit Consequence on Score Distribution..................39

5.CONCLUSION....................................................... 77 
BIBLIOGRAPHY.

.81 


\section{LIST OF TABLES}

Table

Page

3.1 Generating Item Parameter Values.

4.1 Empirical Type I Error Rate for RISE, PARSCALE's $G^{2}$ and IRTFIT's $S$ - $X^{2}$ Statistic, Normal Ability Distribution, 3PLM/2PLM/GPCM Model

Combination.

4.2 Empirical Type I Error Rate for RISE, PARSCALE's $G^{2}$ and IRTFIT's $S$ - $X^{2}$ Statistic, Normal Ability Distribution, 3PLM/2PLM/GRM Model

Combination.

4.3 Empirical Detection Rate for RISE, PARSCALE's $G^{2}$ and IRTFIT's $S$ - $X^{2}$ Statistic, Normal Ability Distribution, 3PLM/2PLM/GPCM Model

Combination.

4.4 Empirical Detection Rate for RISE, PARSCALE's $G^{2}$ and IRTFIT's $S$ - $X^{2}$ Statistic, Normal Ability Distribution, 3PLM/2PLM/GRM Model

Combination.....

4.5 Empirical Type I Error Rate for RISE, PARSCALE's $G^{2}$ and IRTFIT's $S$ - $X^{2}$ Statistic, Negatively-Skewed Ability Distribution, 3PLM/2PLM/GPCM Model

Combination.

4.6 Empirical Type I Error Rate for RISE, PARSCALE's $G^{2}$ and IRTFIT's $S$ - $X^{2}$ Statistic, Negatively-Skewed Ability Distribution, 3PLM/2PLM/GRM Model

Combination. .46

4.7 Empirical Detection Rate for RISE, PARSCALE's $G^{2}$ and IRTFIT's $S$ - $X^{2}$ Statistic, Negatively-Skewed Ability Distribution, 3PLM/2PLM/GPCM Model Combination.

4.8 Empirical Detection Rate for RISE, PARSCALE's $G^{2}$ and IRTFIT's $S$ - $X^{2}$ Statistic, Negatively-Skewed Ability Distribution, 3PLM/2PLM/GRM Model

Combination.

4.9 Empirical Type I Error Rate for RISE, PARSCALE's $G^{2}$ and IRTFIT's $S$ - $X^{2}$ Statistic, Positively-Skewed Ability Distribution, 3PLM/2PLM/GPCM Model

Combination.

4.10 Empirical Type I Error Rate for RISE, PARSCALE's $G^{2}$ and IRTFIT's $S$ - $X^{2}$

Statistic, Positively-Skewed Ability Distribution, 3PLM/2PLM/GRM Model

Combination.

4.11 Empirical Detection Rate for RISE, PARSCALE's $G^{2}$ and IRTFIT's $S$ - $X^{2}$ Statistic, Positively-Skewed Ability Distribution, 3PLM/2PLM/GPCM Model

Combination 
4.12 Empirical Detection Rate for RISE, PARSCALE's $G^{2}$ and IRTFIT's $S$ - $X^{2}$ Statistic, Positively-Skewed Ability Distribution, 3PLM/2PLM/GRM Model

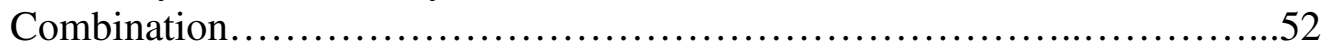

4.13 The difference of the difference of score distributions before and after replacement in Simulation Study....

4.14 The difference of the difference of TCCs before and after replacement in Simulation Study.

4.15 Misfitting Items Detected by Each Fit Statistic at Each Sample Size in Empirical Study

4.16 The difference of the difference of score distributions before and after replacement in Empirical Study .56 


\section{LIST OF FIGURES}

$\begin{array}{lll}\text { Figure } & \text { Page }\end{array}$

3.1 Generating Empirical ICCs.................................................. 32

4.1 Score Distribution Before and After Replacement of Misfitting Items Detected by RISE..............................................................

4.2 Score Distribution Before and After Replacement of Misfitting Items Detected by

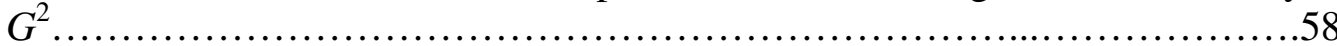

4.3 Score Distribution Before and After Replacement of Misfitting Items Detected by $S-X^{2}$

4.4 TCCs Before and After Replacement of Misfitting Items Detected by

RISE.

4.5 Misfitting Items Detected by RISE at Sample Size $500 \ldots \ldots \ldots \ldots \ldots \ldots \ldots \ldots \ldots \ldots \ldots$

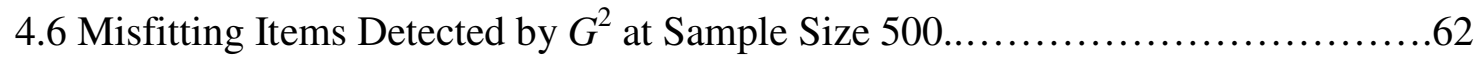

4.7 Misfitting Items Detected by RISE at Sample Size 2,000....................63

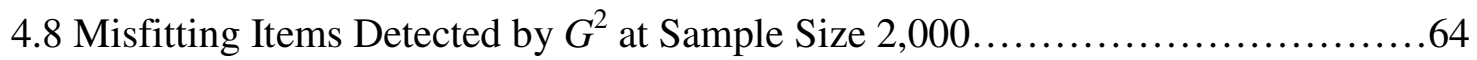

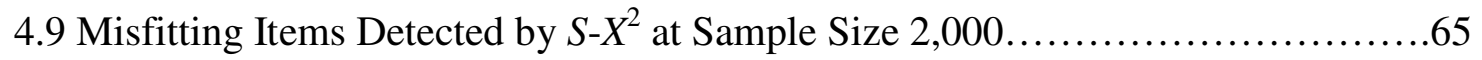

4.10 Misfitting Items Detected by RISE at Sample Size 10,000...................66

4.11 Misfitting Items Detected by $G^{2}$ at Sample Size 10,000.....................67

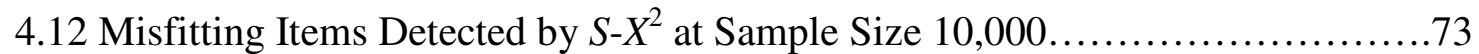

4.13 Score Distribution Before and After Replacement of Misfitting Items Detected by

Three Fit Statistics at Sample Size 500.................................74

4.14 Score Distribution Before and After Replacement of Misfitting Items Detected by

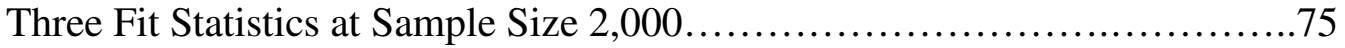

4.15 Score Distribution Before and After Replacement of Misfitting Items Detected by

Three Fit Statistics at Sample Size 10,000.................................76 


\section{CHAPTER 1}

\section{INTRODUCTION}

\section{$\underline{1.1 \text { Background }}$}

Item response theory (IRT) is a powerful scaling technique that provides appealing features such as the invariance of item and ability parameter values. Because of the attractive features IRT provides, parametric IRT models are used for a variety of measurement applications such as constructing tests, equating test scores, estimating proficiency levels, identifying differential item functioning (DIF), and selecting items for computerized adaptive testing (Embretson \& Reise, 2000; Hambleton, Swaminathan, \& Rogers, 1991). However, the success of specific IRT applications and the advantages of item response models are obtained when the fit between the model and the test data is satisfactory. Thus, it is crucial to understand the possible reasons for misfit and evaluate model-data fit as part of the process of validating the use of IRT models.

There are two general sources of misfit in IRT models: (1) violation of model assumptions pertaining to dimensionality/local independence and (2) failure to capture model-data relationships. For the most commonly-used IRT models to be applicable, the item pool must produce item responses that are predominately unidimensional. Fitting a unidimensional model to multidimensional data may produce model misfit that may have a consequential impact on the test results. Failure to capture model-data relationships is a specific type of assumption violation in which the model does not accurately reflect the true probabilities of a correct response (or does not accurately reflect the true probabilities of responding in a particular category for polytomous item responses). There are a few reasons why the model may not accurately reflect the true item characteristic curve (ICC). For example, for a multiple-choice item, the item may 
contain multiple correct answers. Also, the wrong model may be selected (e.g., using a model that does not consider the influence of guessing on multiple choice test items).

\section{$\underline{1.2 \text { Statement of the Problem }}$}

While many of the technical challenges that arise when applying IRT models to educational and psychological data have been resolved to some extent (e.g., model parameter estimation), the assessment of model fit remains a major hurdle to overcome for effective implementation (Hambleton \& Han, 2004). Various methods and approaches have been suggested for detecting model misfit. Traditional methods for assessing goodness-of-fit in IRT are based on Pearson-type $\chi^{2}$ statistics which are most widely used to assess model fit (e.g., Yen's (1981) $Q_{1}$ statistic). However, there are several drawbacks to using Pearson $\chi^{2}$ statistics in assessing model fit. The two most common critiques about the $\chi^{2}$-based statistics are first, they are sensitive to sample size (Hambleton \& Swaminathan, 1985). The distribution theory of $\chi^{2}$ statistics, as well as most other formal tests of fit, is a large-sample theory. When the sample size is large, the statistical test rejects just about every model since with large sample sizes, statistical power is available to detect even very small model-test data discrepancies, and no statistical model for educational testing data is ever going to be strictly correct (i.e., the model will not reflect the true ICC exactly for all ability levels). Therefore, the first criticism is not necessarily because the $\chi^{2}$-based statistics are sensitive to sample size but that the hypothesis being tested is meaningless since it is always false (Cohen, 1994). Second, several popular fit statistics (e.g., $G^{2}$ and Yen's $Q_{1}$ ) are not distributed as a $\chi^{2}$. One reason is that the examinees are grouped based on IRT $\theta$ estimates, which contain error. Particularly speaking, parameter values are never known so the estimators of the parameters are treated as true values, which violates the 
assumption underlying the $\chi^{2}$ distribution (Stone $\&$ Zhang, 2003). Another reason is that the degrees of freedom are in question (Orlando \& Thissen, 2000). As a result, the Type I error rate is inflated, in other words, many valid items are falsely identified as misfitting when in fact the model fits the data (Orlando \& Thissen, 2000; Reise, 1990; Glas \& Falcon ,2003; Stone \& Hansen, 2000; DeMars, 2005).

Due to the limitations of $\chi^{2}$-based statistical methods for assessing model fit, it is prudent that another fit statistic be developed that avoids or limits the previous criticisms. This dissertation will focus on studying an approach for assessing model-fit proposed by Douglas \& Cohen (2001) that compares a nonparametrically-derived ICC to the parametric ICC. This nonparametric approach, hereafter referred to as RISE, has exhibited controlled Type I error rates and adequate power in the dichotomous and polytomous case and provides a graphical representation of the misfit that may be used to judge the severity of the misfit (Wells \& Bolt, 2008; Li \& Wells, 2006; Liang \& Wells, 2009, Liang \& Wells, 2008). Beyond its attractive statistical properties, an additional advantage of the nonparametric approach is that it provides a convenient graphical representation of model misfit. This feature can provide the researcher or practitioner visual guidance about the location and type of misfit.

Concluding that a model is misfitting according to the hypothesis test allows a test developer to conclude that the model does not fit exactly in the population. However, it is possible that a misfitting model is still useful in that it approximates the true ICC very closely and that the consequences of the misfit are minimal. Therefore, it is important to consider the consequences of the misfit prior to rejecting the use of a model or item. In other words, it is worthwhile to investigate whether the misfit of the model has substantial practical consequences for the particular situation. The issue of evaluating practical consequences of model misfit has been given little attention in the 
model checking literature in IRT (Hambleton \& Han, 2004). However, it deserves more attention because it is possible that a statistical test indicates misfit while discrepancies between the test data and predictions from a model are of no practical consequence (van der Linden \& Hambleton, 1997). Thus, this dissertation will also evaluate the importance of the practical consequences of model misfit together with statistical analyses and diagnostic plots for a fuller picture of fit assessment.

\subsection{Objectives of the Study}

The purposes of this study are to (a) review the currently used goodness-of-fit procedures, (b) further examine the promising fit procedure, RISE, (c) compare the statistical properties of RISE with that of the commonly used goodness-of-fit procedures, and (d) investigate how RISE may be used to examine the consequences of model misfit.

\subsection{Outline of the Study}

This study consists of five chapters. In this first chapter, the importance of model-data fit, an introduction of model-data fit problems, the purposes of the study and the outline of the study have been described. Chapter 2 presents a literature review of the IRT models in which the fit statistics will be applied, including RISE, and the comparative research on currently used goodness-of-fit test statistics. Chapter 3 describes the method for the study and also the design of the simulation studies that are conducted. Results will be summarized in Chapter 4. Finally, Chapter 5 will discuss the importance of the results and future research. 


\section{CHAPTER 2}

\section{REVIEW OF LITERATURE}

This chapter begins with an introduction to assumptions of commonly used IRT models as well as a description of the models that will be used in the current dissertation. The fit statistics will then be briefly described followed by findings from empirical research on the fit statistics. Finally, based on the literature, the reasons for further exploring the fit statistic, RISE, are discussed.

\section{$\underline{2.1 \text { IRT Assumptions and Models }}$}

- IRT Models for Dichotomously Scored Data

The three most popular unidimensional IRT models are the one-, two-, and three-parameter logistic models (1PLM, 2PLM and 3PLM, respectively). They have similar conceptual and mathematical formulations. The 3PLM may be expressed as follows:

$$
P_{i}(\theta)=c_{i}+\left(1-c_{i}\right) \frac{\exp \left[D a_{i}\left(\theta-b_{i}\right)\right]}{1+\exp \left[D a_{i}\left(\theta-b_{i}\right)\right]},
$$

where $P_{i}(\theta)$ is the probability that an examinee with ability $\theta$ answers item $i$ correctly, $a_{i}$ is the slope (item discrimination) parameter, $b_{i}$ is the location (item difficulty) parameter, $c_{i}$ is the lower asymptote (pseudo-chance-level) parameter, and $D$ is a scaling parameter. When $\mathrm{D}$ is 1.702 , the probabilities will be within 0.01 of the normal ogive model.

The 2PLM and 1PLM are based on constraints or restrictions of the 3PLM. For example, the 2PLM sets the $c$-parameter to zero for all items. The 1PLM sets the $c$-parameter to zero and constrains the $a$-parameters to be equal for all items on an assessment.

- IRT Models for Polytomously Scored Data 
1. Graded Response Model. The graded response model, developed by Samejima (1969), is appropriate to use when item responses are ordered categorical responses. For example, when Likert-type items are applied in surveys or questionnaires, GRM can be used to analyze those items. Two steps are needed to obtain the probability that an examinee responds to a particular category. The first step in estimating response probabilities involves computation of operating characteristic curves (OCCs), or in other words, measuring the probability that an examinee's response falls in or above a particular category given $\theta$. The OCCs are an extension of the 2PLM and may be expressed as follows:

$$
P_{i j}^{*}(\theta)=\frac{\exp \left[a_{i}\left(\theta-b_{i j}\right)\right]}{1+\exp \left[a_{i}\left(\theta-b_{i j}\right)\right]},
$$

where $j=1, \ldots, m_{i}, m_{i}$ is equal to number of categories minus $1 ; P_{i j}{ }^{*}(\theta)$ is the probability of a randomly chosen examinee with proficiency of $\theta$ scoring $x$ or above on item $i ; a_{i}$ is the slope (item discrimination) parameter for item $i . \quad b_{i j}$ is the threshold (item difficulty) parameter, which is commonly interpreted as the trait level to respond in or above the particular threshold $j$ of item $i$ with 0.5 probability. GRM is an extension of 2PLM and belongs to "difference models." Once OCCs are estimated, the actual category response curves (CRCs), which represent the probability of scoring in a particular category, are calculated by subtraction between two adjacent OCCs:

$$
P_{i j}(\theta)=P_{i j}{ }^{*}(\theta)-P^{*}{ }_{i(j+1)}(\theta) .
$$

2. Generalized Partial Credit Model. Muraki (1992) developed the generalized partial credit model (GPCM) to be used for polytomous data that are based on completing steps (e.g., partial-credit items). The GPCM, classified as a “divide-by-total" model, may be expressed as follows: 


$$
P_{i x}(\theta)=\frac{\exp \left[\sum_{j=0}^{x} a_{i}\left(\theta-b_{i j}\right)\right]}{\sum_{r=0}^{M} \exp \left[\sum_{j=0}^{r} a_{i}\left(\theta-b_{i j}\right)\right]},
$$

where $\sum_{j=0}^{0} a_{i}\left(\theta-b_{i j}\right) \equiv 0$. In the GPCM, $b_{i j}$ is interpreted as the intersection (difficulty) parameters where one category response is more likely to happen than previous responses given that the examinee has reached the previous step. Thus, $b_{i j}$ is not necessarily ordered as occurs in the GRM. For example, if a GPCM item has 5 score categories, there are 4 difficulty parameters with each value at $b_{1}=0.2, b_{2}=-1.3, b_{3}=$ 1.7, $b_{4}=-0.5$, which indicate that the difficulty levels from easiest to hardest are step 2 , step 4, step 1 and step 3 . In addition, when the $a$ parameter is constrained to be equal across items, the GPCM simplifies to the partial credit model (PCM; Masters, 1982).

\section{- Assumptions of IRT Models}

Three important assumptions must be met before the previously IRT models are employed. The first assumption, unidimensionality, states that one ability is measured in a test. Although unidimensionality will not be strictly satisfied since there a several factors that influence test performance, IRT models are still useful as long as there is one predominate factor underlying the test data. The second assumption is local independence which states that the items are uncorrelated after controlling for the ability on the test. A third assumption is placed on the ICC shape. To apply a parametric model, it is assumed that the shape of the true ICC is the same as that posited by the parametric model.

\section{$\underline{2.2 \text { Model-Data Fit Procedures }}$}

A general strategy for evaluating model fit at the item level involves comparing 
observed data with model-predicted expectations (Hambleton \& Han, 2005; Hambleton, Swaminathan, \& Rogers, 1991; Rogers \& Hattie, 1987). Both statistical significance tests and graphical analyses based on the residuals can be used for this purpose. Broadly defined, a goodness-of-fit study is the evaluation of the similarity between observed and expected (predicted) outcomes. Within the context of IRT, this typically involves (1) estimating the parameters of an IRT model, (2) using those parameter estimates to predict, by way of the IRT model, examinee response patterns, and (3) comparing the predicted response patterns to actual observed examinee response patterns.

- Traditional Method: $\chi^{2}$-Based Fit Statistics

Many of the traditional $\chi^{2}$-based methods for assessing model fit are based on the following steps:

1. Estimate item and ability parameters.

2. Place examinees into groups along the ability scale using proficiency estimates.

3. Construct an observed score response distribution for each ability group by cross-classifying examinees using their ability estimates and score responses.

4. Form an expected score response distribution across score categories using item parameter estimates and ability estimates representing each ability group.

5. Compare observed and expected score response distributions using a $\chi^{2}$ fit statistic or examination of residuals.

1. Yen's $\boldsymbol{Q}_{1}$. Yen's (1981) $Q_{1}$ index, proposed for use with dichotomous items, is calculated as follows:

$$
Q_{1}=\sum_{j=1}^{10} \frac{N_{j}\left(O_{i j}-E_{i j}\right)^{2}}{E_{i j}\left(1-E_{i j}\right)},
$$


where $N_{j}$ is the number of examinees in ability interval $j$; $O_{i j}$ is the observed proportion of examinees in interval $j$ who answer item $i$ correctly; and $E_{i j}$ is the probability based on the model in interval $j$ answering item $i$ correctly. The number of intervals is fixed to be 10 (although, if the expected frequency in an interval is less than five, then the groups may be combined so that the expected frequency is greater than 5). Under the null hypothesis, Yen's $Q_{1}$ is distributed as a $\chi^{2}$ with degrees of freedom equal 10 minus the number of parameters being estimated.

2. Likelihood Ratio Test. The likelihood ratio test fit statistic (McKinley \& Mills, 1985), denoted $G^{2}$, is reported by two commonly used commercial IRT software packages, BILOG-MG (Zimowski, Muraki, Mislevy \& Bock, 2003) and PARSCALE (Muraki \& Bock, 2003). $G^{2}$ is calculated for item $i$ as follows:

$$
G^{2}=2 \sum_{h=1}^{n_{g}}\left[r_{h i} \log \frac{r_{h i}}{N_{h} P_{i}\left(\overline{\theta_{h}}\right)}+\left(N_{h}-r_{h i}\right) \log \frac{N_{h}-r_{h i}}{N\left[1-P_{i}\left(\overline{\theta_{h}}\right)\right]}\right],
$$

where $n_{g}$ indicates the number of intervals; $r_{h i}$ represents the observed frequency of correct responses for item $i$ in interval $h ; N_{h}$ is the number of individuals in interval $h$; and $P_{i}\left(\bar{\theta}_{h}\right)$ refers to the model-predicted proportion correct for item $i$ at $\bar{\theta}_{h} . \bar{\theta}_{h}$ is the average of theta estimates in interval $h . G^{2}$ is distributed under the null hypothesis approximately as a $\chi^{2}$ with degrees of freedom equal to the number of intervals. $G^{2}$ is basically a log-likelihood based statistic in which examinees are grouped into ability intervals based on their ability estimates, and then the empirical proportion correct against the model-based proportion correct within that interval is evaluated, accumulating across intervals.

3. Orlando and Thissen's $\boldsymbol{S}-\boldsymbol{X}^{\mathbf{2}}$. Orlando and Thissen (2000) addressed the weakness of Yen's $Q_{1}$ statistic by grouping examinees based on their number correct 
(NC) scores instead of their model-based ability estimates. The fit statistic, $S$ - $X^{2}$, based on Yen's (1981) $Q_{1}$ statistic, is approximately distributed as a $\chi^{2}$ with $G$ - $m$ degrees of freedom, where $m$ indicates the number of item parameters estimated and $G$ is the number of score groups ( $G=n-1$ when no groups have been collapsed, where $n=$ number of score points). $S$ - $X^{2}$ has been extended to test the fit of polytomous models (Bjorner, Smith, Stone \& Sun, 2007; Kang \& Chen, 2008). The general formula for testing the fit of a polytmous or dichotomous items is as follows:

$$
S-X^{2}=\sum_{t=t_{\min }}^{t_{\max }} \sum_{c_{i}=1}^{m_{i}} \frac{\left(O_{t c i}-E_{t c i}\right)^{2}}{E_{t c i}},
$$

where $t$ is sum score, $m$ is number of categories, $O$ is observed frequency and $E$ is expected frequency, $i$ is item. Before calculating $S-X^{2}$, cells are collapsed to achieve expected cell frequencies above a certain number in order to avoid sparse expected frequencies.

4. Lagrange Multiplier (LM) Test. Glas (1998) proposed a Lagrange Multiplier (LM) procedure to evaluate item and ability parameter estimates for 2PLM and 3PLM. The rationale of LM tests is to test a restricted model against a more general alternative, where the restricted model is derived from the general model by imposing constraints on one or more parameters. The LM test is based on the evaluation of the first-order partial derivatives of the log-likelihood function of the general model, evaluated using the maximum likelihood estimates of the restricted model (Glas, 1998). As suggested by Glas, one advantage of using LM method for evaluation of item fit is that the asymptotic distribution of the statistics involved follows directly from asymptotic theory (Glas \& Falcón, 2003).

- Graphical Displays of Model Data Fit 
In contrast to statistical analyses, the discrepancy between the model-based expectations and the observed data (i.e., residuals) may be examined visually via graphical representations to examine model fit. Such graphical displays, while less objective in that they do not provide error rate control as opposed to a strict statistical test, have proven to be useful in evaluating the complex relationship between model and data (Hambleton, Swaminathan, \& Rogers, 1991). Residual plots provide information on the location and magnitude of possible misfit. Graphical displays are often best used in conjunction with statistical tests in that the test provides information regarding whether the model fits and the practical significance of the misfit.

- Methods Involving Comparison of Observed Data with Simulated Data

Hambleton and Han (2005) suggested assessing the goodness-of-fit of commonly used IRT models by comparing the observed score distribution to the distribution from simulated data according to the selected model, and using Komogrov-Smirnov test as a descriptive indicator of the difference in the distributions. The comparison of the observed and simulated score distribution can be based on all examinees to evaluate fit at the test level, or conditional on the observed performance of a particular item to evaluate fit at the item level. To compute the conditional test score given ability, the Lord-Wingersky recursive formula (Lord \& Wingersky, 1984), and an extension of the Lord-Wingersky formula proposed by Wang, Kolen, and Harris (2000) are applied. Readers are referred to Wainer and Mislevy (1990), Kingston and Dorans (1985), Ankenmann (1994), Sinharay (2005) for illustrative examples of model data fit.

\section{- Bayesian Approach}

Under Bayesian framework, the posterior predictive model checking (PPMC) technique was proposed by Guttman (1967). It makes use of the posterior predictive 
distribution of replicated data as a reference distribution for the observed data. In practice, data sets are simulated by generating parameter values from the predictive distribution. If the simulations and the data differ systematically, it is an indication of a potential model misfit.

Donoghue and Hombo $(1999,2001)$ derived a distribution for the fit statistic $D H Q$ for dichotomous items. Their method uses posterior probabilities in computing item fit. The fit statistics proposed by Donoghue and Hombo (1999) are based on the assumption that the item parameters are fixed and known. Under this restrictive assumption, they demonstrated that the fit measure is asymptotically distributed as a quadratic form of a normal random variable. Donoghue and Hombo (2001) extended this method for use with polytomous data, and showed that the statistic is a quadratic form of normals as well in the extended application.

- Nonparametric Approach (RISE)

Douglas and Cohen (2001) proposed an approach in which a nonparametrically-based ICC is compared to the best-fitting parametric ICC. If the two ICCs differ substantially, then the parametric model is considered to be misfitting. The concept underlying this approach is based on the fact that a nonparametrically-derived ICC (e.g., kernel-smoothed estimates) provides a reasonable estimate to the true ICC under a weak set of assumptions (Douglas, 1997). Kernel smoothing is a popular nonparametric method developed by Ramsay (1991) and is based on the concept of local averaging. Local averaging is useful in estimating points on an ICC because $P(u=1 \mid \theta)=E(Y \mid \Theta=\theta)$. Therefore, a reasonable estimate of $P(u=1 \mid \theta)$ can be obtained by taking a weighted average of the response variable $Y$ in which responses for examinees whose $\theta$-value is close to a focal ability level, referred to as an evaluation point, contribute most heavily in estimating $P(u=1 \mid \theta)$. Since the 
nonparametric approach imposes fewer restrictions on the shape of the ICC, it is concluded that the parametrically-based model is incorrect if it differs from the nonparametric ICC substantially. The following describes the steps used in the procedure of RISE.

- Estimate the category curve(s) using kernel smoothing, which is computed as follows:

$$
P\left(\theta_{j}\right)=\frac{K\left[\frac{\hat{\theta}_{i}-\theta_{j}}{h}\right] Y_{i}}{\sum_{i=1}^{n} K\left[\frac{\hat{\theta} i-\theta j}{h}\right]},
$$

where $K$ is Gussian kernel, $\theta_{j}$ is evaluation point, $\theta_{i}$ is theta estimate, $Y_{i}$ is examinee's response.

- Compute the optimal item parameter values for the parametric model of interest.

- Summarize the difference between the nonparametric and parametrically-based step ICCs for each item using the following statistic:

$$
R I S E_{i}=\sqrt{\frac{\sum_{k=1}^{K}\left[\frac{\left.\sum_{q=1}^{Q}\left(\hat{P}_{q k}-\hat{P}_{q k}^{n o n}\right)^{2}\right]}{Q}\right]}{K-1}}
$$

where $\hat{P}_{q k}$ and $\hat{P}_{q k}^{n o n}$ represent points on the ICCs for the model-based and nonparametric methods for each category; $Q$ is the number of evaluation points (e.g., $Q=100)$; and $K$ is the total number of categories. In the dichotomous case, $K=2$.

- To determine the significance level of the observed statistic, the following parametric bootstrapping procedure is performed to construct an empirical distribution for $R I S E_{i}$ under $H_{0}$ (i.e., model fit). 
1. Simulate item response data using the optimal item parameter estimates for each model obtained previously and $N$ As sampled from a standard normal distribution.

2. Estimate the category curves and ICCs for the simulated data using kernel smoothing.

3. Compute the optimal values for item parameter estimates for each model, as described previously.

4. Compute $R I S E_{i}$ for each item using equation (9).

5. Repeat steps 1 to $4 M$ times (e.g., $M=500)$.

If the observed $R I S E_{i}$ is greater than the $95^{\text {th }}$ percentile of the constructed $R I S E_{i}$, the conclusion is the model does not fit the data. In addition, for the nonparametric family, Wells and Bolt (2008), Li and Wells (2006), and Wells (2009), and Liang and Wells (2008) described the graphical representation of model misfit between nonparametric and parametric ICC to provide visual guidance about the location, type of misfit and magnitude of misfit.

\section{$\underline{2.3 \text { Empirical Research on Fit Indices }}$}

In this section, a number of studies comparing the fit procedures are reviewed. The Type I error rate and power are two commonly used criteria that provide useful information regarding the statistical properties of a test statistic. As for graphical analysis, there are usually no clear criteria because it is more subjective and used as a supplement to significance tests. Model misfit consequence is one important issue as part of the fit procedure; therefore, a number of studies on this topic are also reviewed here. A summary of results from these studies is provided afterwards.

For dichotomous items, McKinley and Mills (1985) compared the performances of traditional goodness of fit statistics in detecting misfit. The fit statistics included were 
Bock's $\chi^{2}$, Yen's $Q_{1}$, Wright and Mead $\chi^{2}$ (Wright $\&$ Mead, 1977; Yen, 1981), and the likelihood ratio $\chi^{2}\left(G^{2}\right)$. Data were simulated to fit four models: 1PLM, 2PLM, 3PLM, and a two-factor linear model. Misfit was examined using an IRT model that was different from the model used to simulate item responses. By comparing Type I error rates, they found that the likelihood ratio statistics yielded the fewest erroneous rejections of fit when the data were simulated and calibrated using the same model. However, given controlled Type I error rates, Bock's (1972) index appeared to yield slightly better performances in terms of correct rejections of fit when the model used to simulate the items differed from the model used to calibrate the items. As a result, the authors could not conclude which measure was the best. Instead, they suggested that the choice of the fit procedures depend on the type of error that is considered to be the more serious error in a particular setting. With regard to the sample size issue, they concluded that sample sizes of 500 to 1000 were likely to yield the best results while a sample of 2000 seemed to make the fit procedures too sensitive.

Orlando and Thissen (2000) conducted a simulation study investigating the Type I error rates and empirical power for their new proposals of item fit statistics for dichotomously scored items. They compared $S-X^{2}$ and $S-G^{2}$ statistics with Yen's $Q_{1}$ and McKinley and Mills' log likelihood ratio statistic. They conducted three conditions in which the simulating and fitting models were identical to compute Type I error rates and three conditions involving model misspecifications to compute power. Results indicated that both Yen's (1981) $Q_{1}$ and McKinley and Mills's (1985) index had very high Type I error rates for dichotomous items, particularly for short tests. With sample sizes of 1,000 , for a nominal $\alpha$ level of 0.05 , empirical Type I error rate was around 0.95 for 10 -item tests, between 0.10 and 0.29 for 40 -item tests, and somewhat lower but still inflated for 80 -item tests. The results also showed that $S-X^{2}$ 
performed better than the other statistics, in that its Type I error rates were close to the specified significance level while the others tended to reject the null hypothesis too often. In another study, Orlando \& Thissen (2003) further investigated the utility of $S-X^{2}$ as a model fit index for the 3PLM. Three types of item misfit were simulated: An item that followed a nonmonotonic shaped; an item in which the probability of answering correctly does not reach 1 (i.e., the upper-asymptote is not 1); an item that exhibits a plateau over middle values of $\theta$ but follows a logistic curve before and after the plateau. The simulation compared the performance of $S-X^{2}$ to that of $Q_{1}-X^{2}$, a Pearson $\chi^{2}$ form of Yen's $Q_{1}$ index. Results indicated that the performance of $S-X^{2}$ was improved with increasing test length and sample size, and was superior to that of $Q_{1}-X^{2}$ under most conditions.

Stone and Zhang (2003) presented results comparing the Type I error rates and empirical power for three alternatives for dichotomously scored items, including Orlando and Thissen's (2000) method, Stone's (2000a) resampling or pseudocounts-based method, and Donoghue and Hombo's (1999) DHQ index. As a frame of reference with traditional approaches to assessing model-data fit, Bock's (1972) $\chi^{2}$ statistic was also examined. They reported excessively unacceptable Type I error rates for Bock's (1972) statistic, which is consistent with previous research. By contrast, the three new approaches appeared to offer promising alternatives to traditional methods. Among the three methods, they found that Stone's method tended to display more power to detect misfit in smaller samples, while Orlando and Thissen's method showed adequate power to detect misfit with a sample size of 2000 under the 2PLM or 3PLM simulated and 1PLM calibrated conditions, and the Donoghue and Hombo's method lacked adequate power at all sample sizes. However, none of the methods showed adequate power in detecting misfit under the 3PLM simulated and 
2PLM calibrated conditions, which corroborates previous research (McKinley \& Mills, 1985; Yen, 1981).

Glas and Falcón (2003) proposed another index based on a Lagrange multiplier (LM) test and compared this index with Orlando and Thissen's (2000) $S-G^{2}$ and the log-likelihood ratio test. The Type I error rate and power of the test of fit of the 3PLM was investigated. The test lengths in the simulation studies were 10, 20 and 40, and the examinee sample sizes were 500,1000, and 4000. The results showed that the overall characteristics of the $S-G^{2}$ test, in terms of the ratio of hits and false alarms, were better than the overall characteristics of the LM test. They suggested that this difference could be due to differences in item parameters. As in Orlando and Thissen's procedure, for this index, examinees are grouped by number-correct scores rather than IRT estimated trait scores which were the grouping basis of the usual log-likelihood ratio index, and the LM index also takes into account the standard errors in the item parameter estimates.

For polytomous items, Stone and Hansen (2000) examined a Pearson's $\chi^{2}$ type index similar to Yen's $Q_{1}$ (1981) or Bock's (1972) $\chi^{2}$ and a log-likelihood ratio index similar to the statistic reported in PARSCALE's. Using true item parameters and true abilities, Type I error rates were somewhat more inflated for the log likelihood ratio index than for Pearson's $\chi^{2}$. However, using estimated abilities and true item parameters, Type I error rates were extremely inflated, especially for short tests. For example, for an eight-item test with a nominal $\alpha$ of 0.05 , the Type I error rate ranged from 0.75 to 1.00 for the selected items displayed. The inflation was smaller with longer tests and for smaller sample sizes. The log likelihood index studied by Stone and Hansen differed somewhat from PARSCALE's index; item parameters were known rather than estimated, and .000001 was added to cells with an expected frequency of 0 , 
compared with PARSCALE's procedure of combining groups so that all cells have an expected frequency of at least 5 .

DeMars (2005) examined the Type I error rates for PARSCALE's fit statistic $G^{2}$ for polytomous items. Using either the GRM or the PCM, data were simulated with 10 and 20 items. Type I error rates were found to be inflated for the shorter test length and for the GRM, and also for the longer test length when the ability distribution was uniform. Given the findings, the conclusion was that the fit index cannot be recommended for short tests.

Schrader, Ansley, and Kim (2004) examined the behavior of $Q_{1}$ and two new indices proposed by Orlando and Thissen (2000), $S-X^{2}$ and $S-G^{2}$, on polytomous data (e.g., constructed-response data) fit by the GPCM. In the study, those indices originally developed for dichotomous data were extended to more general forms for use with polytomous data. The misfitting items were from simulating a two-dimensional version of GPCM. They found that the Type I error rates from $S-X^{2}$ were very close to the significance level for various test lengths, while the $Q_{1}$ indices had inflated Type I error rates for shorter test lengths and more reasonable rates for the longest test. However, none of the selected indices had sufficient power to detect misfit under most of the two dimensional conditions. Based on the results, the authors suggested that the $Q_{1}$ indices were not useful in testing model fit, either due to inflated Type I error rates or insufficient power. Most of their results for polytomous data were found to be consistent with results found in Orlando and Thissen's studies for dichotomous data.

For a mix of dichotomous and polytomous items, Chon, Lee, and Ansley (2007) compared three different approaches for assessing IRT model-data fit using real test data containing both multiple choice and constructed response items. In order to evaluate fit for mixed data, they compared PARSCALE's $G^{2}$ as a traditional approach 
with the generalized forms of Orlando and Thissen's $S-X^{2}$ and $S-G^{2}$. Some inconsistencies were found between traditional and new indices for assessing the fit of IRT models based on a mixed-format test. It was reported that the new indices indicated considerable better model fit than the traditional indices. Thus, the results from Chon, Lee, and Ansley (2007) provide a preliminary investigation for the model-data fit procedures.

Due to the unappealing features of statistical tests of misfit (e.g., a significant result does not imply a practically meaningful misfit), there is an urgent need for graphical procedures and displays for assessing model fit for both dichotomous and polytomous data. Some commercial IRT estimation software includes raw residual plots in the standard output—e.g., BILOG-MG (Zimowski, Muraki, Mislevy, \& Bock, 2003). Among the widely used commercial software packages, BILOG-MG is one of the few that plots high quality graphs for identifying the fit in IRT models. The residual plots in BILOG-MG are proving to be popular and helpful in explaining model fit.

Unfortunately, such fit plots are not available in PARSCALE (Mislevy \& Bock, 2003), one of the most popular IRT software packages, which can handle polytomously-scored items.

Liang, Han and Hambleton (2009) developed software called ResidPlots-2 which visually represents the discrepancy between the model-based expectations and the observed data. It provides a powerful tool for graphical residual analyses and is a valuable advance in convenience and flexibility for researchers and practitioners wishing to do graphical residual analysis. The advantages of this software include several features. First, it supports the most widely used IRT models including three dichotomous models (1PLM, 2PLM, 3PLM) and three polytomous models (GRM, GPCM, NRM). Second, it provides considerable flexibility with respect to the number 
and size of the intervals for which the residuals are computed. Users are able to decide the number of intervals, choose the interval size to provide equal width or equal frequency, select the location of the data plot in each interval, eliminate intervals at the lower or higher end of the proficiency scale if desired, etc. Third, it allows users to decide what type of error bars they wish to have displayed. Users can specify the number of standard errors represented by the error bars (e.g., $2 \mathrm{SE}$ ). Fourth, it provides three sets of plots: (1) the item level, raw residual plots and standardized residual plots with error bars; (2) the test level, ResidPlots-2 can show standardized residual distributions (PDF and CDF) with corresponding tables, item fit plots and score fit plots from both empirical and simulated data; and (3), observed test score distributions and predictive score distributions (PDF and CDF) are produced as well. The predictive test score distribution is based on simulation data generated from item and ability parameter estimates from the observed data. Fifth, some tables including basic item fit and person fit statistics and summaries of residuals are provided.

While the nonparametric approach, RISE, has exhibited controlled Type I error rates and adequate power under the studied conditions, a further promising advantage of this nonparametric approach demonstrated that it provides a graphical inspection of possible misfit. Wells and Bolt (2008) and Li and Wells (2006) examined RISE's performance on 2PLM and GRM, respectively. Recently, Liang and Wells (2009) applied this nonparametric approach, RISE, to test the fit of the GPCM. In their study, the empirical Type I error rate and power of RISE were assessed for various test lengths (10-, 20-, and 40 items), sample sizes (500, 1000 and 2000), and type of assessment. What's more, Liang and Wells (2008) further explored the statistical properties of the proposed fit statistic, RISE on a mixed format test that included the 3PLM, 2PLM, and GPCM. The empirical Type I error rate and power of RISE were assessed for various 
sample sizes (2000, 3000 and 5000). The results were compared to $G^{2}$ in PARSCALE (Muraki \& Bock, 2003) and $S-X^{2}$ (Orlando \& Thissen, 2000). The conclusion from the two papers was that compared to the two popular parametric methods, PARSCALE'S $G^{2}$ (Muraki \& Bock, 2003) and $S-X^{2}$ (Orlando \& Thissen, 2000), RISE better controlled Type I error rate and exhibited more power than $S-X^{2}$ and $G^{2}$.

\section{$\underline{2.4 \text { Summary }}$}

The review of literature in this chapter highlights the limitations of statistical tests. Generally speaking, such tests tend to be narrowly focused on a particular aspect of the relationship between the model and the data, often summarizing the evaluation using a descriptive statistic or test result. Specifically speaking, there are three general problems with several of the commonly-used model fit statistics. First, it is well known that several of the statistics (e.g., $G^{2}$ and Yen's $Q_{1}$ ) are not distributed as a $\chi^{2}$ because the examinees are grouped based on IRT $\theta$ estimates, which contain error, and because the degrees of freedom are in question (Orlando \& Thissen, 2000); as a result, the Type I error rate is inflated under many conditions (when the Type I error rate is inflated, many valid items are falsely identified as misfitting when in fact the model fits the data). Second, even when the fit statistic is able to control the Type I error rate, at least for most test lengths (e.g., Orlando and Thissen's $S$ - $X^{2}$ ), there is no accompanying method of assessing how poorly a model fits given a statistic that indicates misfit. This is problematic when assessing fit using very large samples because trivial misfit often leads to the rejection of the hypothesis that the model fits the data exactly in the population. Third, there are a limited number of statistical tests that can assess the fit of multiple IRT models used in a mixed format test. This is particularly problematic since many large-scale assessments utilize both dichotomous and polytomously-scored data. Graphical residual analyses, on the other hand, have 
proven to be useful in evaluating the complex relationship between model and data (Hambleton, Swaminathan, \& Rogers, 1991). The problem of relying on graphical evidence only is that it is less objective than a statistical test; as a result, it can be difficult to judge whether an item is providing reasonable fit or if the item misfit is practically meaningful. Therefore, it is helpful to use both statistical tests and graphical analysis to determine how well a model fits.

As discussed previously, the nonparametric approach for assessing fit appears to be promising due to the controlled Type I error rate, sufficient power to detect misfit, and the convenient graphical display of possible misfit that is available. The promising results of RISE support further study of the method in different testing contexts (e.g., non-normal ability distributions) and in exploring its use in examining the potential consequences of the misfit. Under these circumstances, the current study extends the use of RISE to more general and comprehensive applications by manipulating variety of factors (e.g., test length, sample size, IRT models, ability distribution). Results (e.g., type I error rate and power) will be compared to $G^{2}$ in PARSCALE (Muraki \& Bock, 2003) and $S$ - $X^{2}$ (Orlando \& Thissen, 2000). In order to demonstrate its advantage of graphical representation of model misfit, some residual plots will be displayed from empirical data. Lastly, the consequences of potential misfit will be explored via the nonparametric approach using empirical data. 


\section{CHAPTER 3}

\section{METHOD}

The nonparametric approach for assessing model fit was examined via a Monte Carlo simulation study and an analysis of empirical data. The Monte Carlo simulation study was performed to examine the empirical Type I error rate and power of the proposed statistic to detect misfitting items in a mixed format test under thirty-six conditions. In addition, the statistical properties of RISE were compared to a commonly reported fit statistic, $G^{2}$, provided by the computer package, PARSCALE (Muraki \& Bock, 2003), and a presently developed fit statistic, $S-X^{2}$, provided by the computer software, IRTFIT (Bjorner, Smith, Stone \& Sun, 2007). An empirical data set was analyzed to explore the use of the nonparametric approach for assessing the practical meaningfulness of the misfit via the hypothesis test provided by RISE, inspection of residuals. Comparison of score distributions and test characteristic curves (TCCs) were also addressed as two aspects of model misfit consequence.

\section{$\underline{3.1 \text { The Procedure of } R I S E}$}

The RISE statistic was implemented for assessing the fit of multiple IRT models in a mixed-format as follows:

- Kernel smoothing was used to estimate the ICC for dichotomous data and OCC or CRC for polytomous data. In order to increase computational speed, the Fast Fourier Transformation (FFT) will be applied (Ramsay, 1991).

- Compute the optimal item parameter values for the parametric model of interest (i.e., 2PLM or 3PLM for dichotomous data, and GRM or GPCM for polytomous item responses). For the 2PLM and GRM, the optimal item parameter values were based on the nonparametric ICC using maximum likelihood estimation, the EM algorithm (Wells \& Bolt, 2008, Li \& Wells, 2006). 
To maximize the log-likelihood, the Newton-Raphson procedure was conducted. However, two modifications were needed. One was to replace the observed proportion of correct response at each theta level by the kernel-smoothed probability at each evaluation point. The other was to compute the weight for each evaluation point instead of each theta value. For the 3PLM, a nonlinear model was fitted with three unknown parameters $(\mathrm{a}, \mathrm{b}, \mathrm{c})$. The nonlinear model used a $\chi^{2}$ merit function (Press, Teukolsky, Vetterling \& Flannery, 1992) which was defined to determine best-fit parameters. The minimization procedure proceeded iteratively until $\chi^{2}$ statistic stopped decreasing. And for the GPCM, least squares were used on the logit deviates (Liang \& Wells, 2009). In other words, although the relationship between $\theta$ and each step ICC was nonlinear, the relationship between the logistic deviate, $\left(\log \left[\frac{P_{k+1}}{P_{k}}\right]\right)$, for each step ICC and $\theta$ was linear allowing the use of least squares to estimate the slope and difficulty for each step ICC.

- Summarize the difference between the nonparametric and parametrically-based step ICCs for each item using the following statistic:

$$
\operatorname{RISE}_{i}=\sqrt{\frac{\sum_{k=1}^{K-1}\left[\frac{\left.\sum_{q=1}^{Q}\left(\hat{P}_{q k}-\hat{P}_{q k}^{n o n}\right)^{2}\right]}{Q}\right]}{K-1}}
$$

where $\hat{P}_{q k}$ and $\hat{P}_{q k}^{n o n}$ represent points on the ICCs for the model-based and nonparametric methods for each category; $Q$ is the number of evaluation points (e.g., $Q=100$ ); and $K$ is the total number of categories. In the dichotomous case, $K=2$. 
- To determine the significance level of the observed statistic, the following parametric bootstrapping procedure was performed to construct an empirical distribution for RISE under $H_{0}$ (i.e., model fit).

1. Simulate item response data using the optimal item parameter estimates for each model obtained previously and $N \quad \theta$ s sampled from a standard normal distribution.

2. Estimate the category curves and ICCs for the simulated data using kernel smoothing.

3. Compute the optimal values for item parameter estimates for each model, as described previously.

4. Compute RISE for each item using equation (10).

5. Repeat steps 1 to $4 M$ times (e.g., $M=500)$.

If the observed $R I S E_{i}$ was greater than the $95^{\text {th }}$ percentile of the constructed $R I S E_{i}$, then we concluded that the model did not fit the data.

\section{$\underline{3.2 \text { Four Factors }}$}

A Monte Carlo simulation study was employed to examine the statistical properties of RISE for a mixed-format test. Four factors were varied: ability distribution, sample size, test length, and models.

Three ability distributions were considered to represent three shapes which are commonly found in practice. In particular, they were normal with mean of 0 and standard deviation of 1 , positively skewed $\left(\gamma_{1}=0.95\right)$ and negatively skewed distributions $\left(\gamma_{1}=-0.95\right)$.

The sample sizes were 500, 2,000 and 10,000 to represent small, medium and large sample sizes. Sample size 2,000 is typically used in many testing programs for anchor items; however, the small sample size 500 and large sample size 10,000 were 
also examined for not only the purpose of generality but also the practical use since most traditional fit statistics do not perform well under these sample sizes which are actually not uncommon in some testing scenario.

Two test lengths that represent a small and relatively large assessment were investigated: 30 and 60 items. Those were typical test lengths in some testing programs.

Oftentimes, large-scale standardized tests, such as those used in statewide assessments, comprise both dichotomous (e.g., multiple-choice and short-answer) and polytomous (e.g., partial credit) item responses used to define the latent theta metric. To represent this context and cover variety of models efficiently, mixed format tests were simulated, so a mixture of binary and polytomous IRT models (3PLM, 2PLM, and GRM//GPCM) were assessed in terms of goodness-of-fit.

The percentage of misfitting items was not considered in the study because this factor does not influence either Type I error or power (Wells \& Bolt, 2008). In summary, there were 36 conditions ( 3 ability distributions X 3 sample sizes $X 2$ test lengths $X 2$ formats).

In addition, the consequence of the misfit was explored by comparing the test distribution based on the raw data (or the nonparametric ICCs) versus the test distribution based on the parametric models. The procedure was illustrated below:

- Pick one simulation data (10,000 by 60$)$ and identify misfitting items based on fit statistics

- Compute observed score distribution (CDF) and expected score distribution $(\mathrm{CDF})$

- Select score cuts and compute the CDF differences at those cuts

- Replace those detected misfitting items by fitting items based on item and ability parameter estimates to form a new dataset 
- Compute observed score distribution (CDF) and expected score distribution (CDF) from the new dataset

- Compute the new CDF differences at the cuts again

- If the new difference was not significantly different from the old difference, then there was no model misfit consequence on those cuts of score distribution.

The true TCCs were compared with the estimated TCCs to illustrate model misfit consequences. The procedure for investigating misfit consequence on TCC is shown below:

- Pick one simulation data (10,000 by 60$)$ and identify misfitting items based on fit statistics

- Compute true TCC and estimated TCC

- Select theta cuts and compute the TCC differences at those cuts

- Replace those detected misfitting items by fitting items based on item and ability parameter estimates to form a new dataset

- Compute true TCC and estimated TCC from the new dataset

- Compute the new TCC differences at the cuts again

- If the new difference was not significantly different from the old difference, then there was no model misfit consequence on those cuts of TCC.

\subsection{Data Generation}

Item response data were generated for each of the thirty-six conditions. The 30-item test consisted of twenty 3PLM items (representing multiple-choice items), five 2PLM items (representing short-answer items), and five GPCM (or GRM) items (representing five-category, polytomous responses) for three sample sizes: 500-, 2,000-, and 10,000-examinees. The item responses were simulated from three ability 
distributions, respectively. Similarly, the 60-item test consisted of forty 3PLM items (representing multiple-choice items), ten 2PLM items (representing short-answer items), and ten GPCM (GRM) items (representing five-category, polytomous responses).

To assess the Type I error rate, $80 \%$ of the 3PLM items (16 items in the 30 -item test, 32 items in the 60 -item test), $80 \%$ of the 2 PLM items ( 4 items in the 30 -item test, 8 items in the 60 -item test) and $80 \%$ of the GPCM (GRM) items (4 items in the 30 -item test, 8 items in the 60 -item test) were simulated from the respective parametric model. The generating item parameter values were obtained from a state-wide test that used a similar test format. Table 3.1 reports the generating item parameter values for the 60 -item test. The values used in the 30 -item conditions were a subset of those used in the 60-item condition.

To assess power, the remaining $20 \%$ of the total items were simulated from empirically-derived ICCs that did not follow their respective parametric models. The ICCs were obtained from a statewide assessment. Figure 1 reports the underlying ICCs for generating misfit based on kernel smoothed estimates for the 60-item test. Note that the percentage of misfitting items does not appear to influence either Type I error rate or power (Wells \& Bolt, 2008), so other percentages were not examined in the study.

One-hundred replications were performed for each of the three sample size conditions. The underlying $\theta$ values were sampled from the three ability distributions.

\subsection{Data Analyses}

FORTRAN code was written and implemented to perform the nonparametric approach to assess misfit and perform the parametric bootstrapping procedure. The computer software package, PARSCALE (Muraki \& Bock, 2003), was used to obtain the fit statistic, $G^{2}$, the computer software, IRTFIT (Bjorner et al.,2007) was used to obtain the fit statistic, $S-X^{2}$. All residual plots and score distribution plots based on 
parametric approach were produced by the computer software ResidPlots-2 (Liang, Han \& Hambleton, 2009).

Simulation Analyses. The empirical Type I error rate and power were assessed for $R I S E$ as well as $G^{2}$ and $S-X^{2}$. The empirical Type I error rate was based on the proportion of items simulated using the three model combinations that were detected as misfitting at $\alpha=.01$ and $\alpha=.05$. Power was assessed based on the proportion of items simulated using the empirically-derived category curves shown at $\alpha=.01$ and $\alpha=.05$. However, power was interpreted for the specific statistic only for conditions in which the Type I error rate was controlled. Score distributions and TCCs before and after replacement of misfitting items were plotted to illustrate model misfit consequence.

Empirical Analyses. An additional advantage of the nonparametric approach, RISE, was the convenient graphical representation of model misfit. This feature can provide visual guidance about the location and type of misfit. The graphical features of the nonparametric approach were explored via the analysis of empirical data. The fit of the three model combinations was tested using RISE as well as $G^{2}$ and $S-X^{2}$. The data came from a large-scale assessment with thirty-two multiple-choice items, four short-answer items, and six partial-credit, five-category, polytomous items at sample sizes of 500, 2,000, and 10,000. Items flagged as misfitting according to the three statistical test statistics were compared. The misfit was further explored via a graphical representation. For each detected misfitting polytomous item, the empirical data and item characteristic curves were aggregated to form one raw residual plot. Model misfit consequence on score distribution was also investigated using the same procedure as the simulation study. 
Table 3.1 Generating Item Parameter Values

\begin{tabular}{|c|c|c|c|c|c|c|c|}
\hline Item & $\alpha$ & $\beta$ & $\gamma$ & $\beta_{1}$ & $\beta_{2}$ & $\beta_{3}$ & $\beta_{4}$ \\
\hline 1 & 1.027 & -0.750 & 0.096 & & & & \\
\hline 2 & 0.985 & -0.660 & 0.084 & & & & \\
\hline 3 & 1.098 & -0.404 & 0.095 & & & & \\
\hline 4 & 1.379 & 0.247 & 0.135 & & & & \\
\hline 5 & 1.429 & -0.850 & 0.094 & & & & \\
\hline 6 & 0.957 & 0.734 & 0.343 & & & & \\
\hline 7 & 1.099 & -0.078 & 0.298 & & & & \\
\hline 8 & 1.268 & 0.122 & 0.221 & & & & \\
\hline 9 & 0.968 & -0.334 & 0.134 & & & & \\
\hline 10 & 1.414 & -0.041 & 0.156 & & & & \\
\hline 11 & 1.464 & -0.382 & 0.306 & & & & \\
\hline 12 & 1.415 & 0.539 & 0.169 & & & & \\
\hline 13 & 0.880 & 0.228 & 0.159 & & & & \\
\hline 14 & 0.882 & -0.674 & 0.158 & & & & \\
\hline 15 & 1.054 & -0.460 & 0.239 & & & & \\
\hline 16 & 1.096 & 0.363 & 0.288 & & & & \\
\hline 17 & 0.996 & -0.161 & 0.193 & & & & \\
\hline 18 & 1.499 & -0.286 & 0.273 & & & & \\
\hline 19 & 1.541 & -0.810 & 0.259 & & & & \\
\hline 20 & 1.724 & -0.634 & 0.258 & & & & \\
\hline 21 & 1.460 & 0.747 & 0.070 & & & & \\
\hline 22 & 0.680 & -0.418 & 0.191 & & & & \\
\hline 23 & 0.959 & -0.300 & 0.284 & & & & \\
\hline 24 & 1.017 & 0.144 & 0.165 & & & & \\
\hline 25 & 1.011 & 0.134 & 0.162 & & & & \\
\hline 26 & 0.948 & -0.298 & 0.281 & & & & \\
\hline 27 & 0.675 & -0.400 & 0.189 & & & & \\
\hline 28 & 1.450 & 0.737 & 0.050 & & & & \\
\hline 29 & 0.721 & -0.629 & 0.255 & & & & \\
\hline 30 & 0.839 & -0.798 & 0.257 & & & & \\
\hline 31 & 1.496 & -0.282 & 0.269 & & & & \\
\hline 32 & 0.995 & -0.151 & 0.189 & & & & \\
\hline 33 & 0.952 & 0.267 & & & & & \\
\hline 34 & 0.985 & 0.060 & & & & & \\
\hline 35 & 1.151 & 0.284 & & & & & \\
\hline 36 & 0.986 & -1.311 & & & & & \\
\hline 37 & 0.876 & 0.145 & & & & & \\
\hline 38 & 0.946 & -0.977 & & & & & \\
\hline 39 & 1.013 & 0.173 & & & & & \\
\hline 40 & 1.202 & -0.004 & & & & & \\
\hline 41 & 1.137 & & & -1.054 & -1.025 & 0.344 & 0.681 \\
\hline 42 & 0.796 & & & -0.090 & -1.277 & 0.053 & 0.215 \\
\hline 43 & 1.213 & & & -0.030 & -0.461 & 1.013 & 0.421 \\
\hline 44 & 1.004 & & & -0.839 & -1.224 & -1.083 & -0.068 \\
\hline 45 & 1.026 & & & -1.035 & -1.060 & 0.267 & 0.534 \\
\hline
\end{tabular}




\begin{tabular}{cccccc}
\hline 46 & 0.724 & -0.010 & -1.255 & 0.023 & 0.210 \\
47 & 0.945 & -0.777 & -1.201 & -1.139 & -0.016 \\
48 & 0.893 & -1.001 & -1.924 & 0.004 & -0.237 \\
& & & & & \\
& & & & & \\
\hline
\end{tabular}


Figure 3.1.Generating Empirical ICCs.
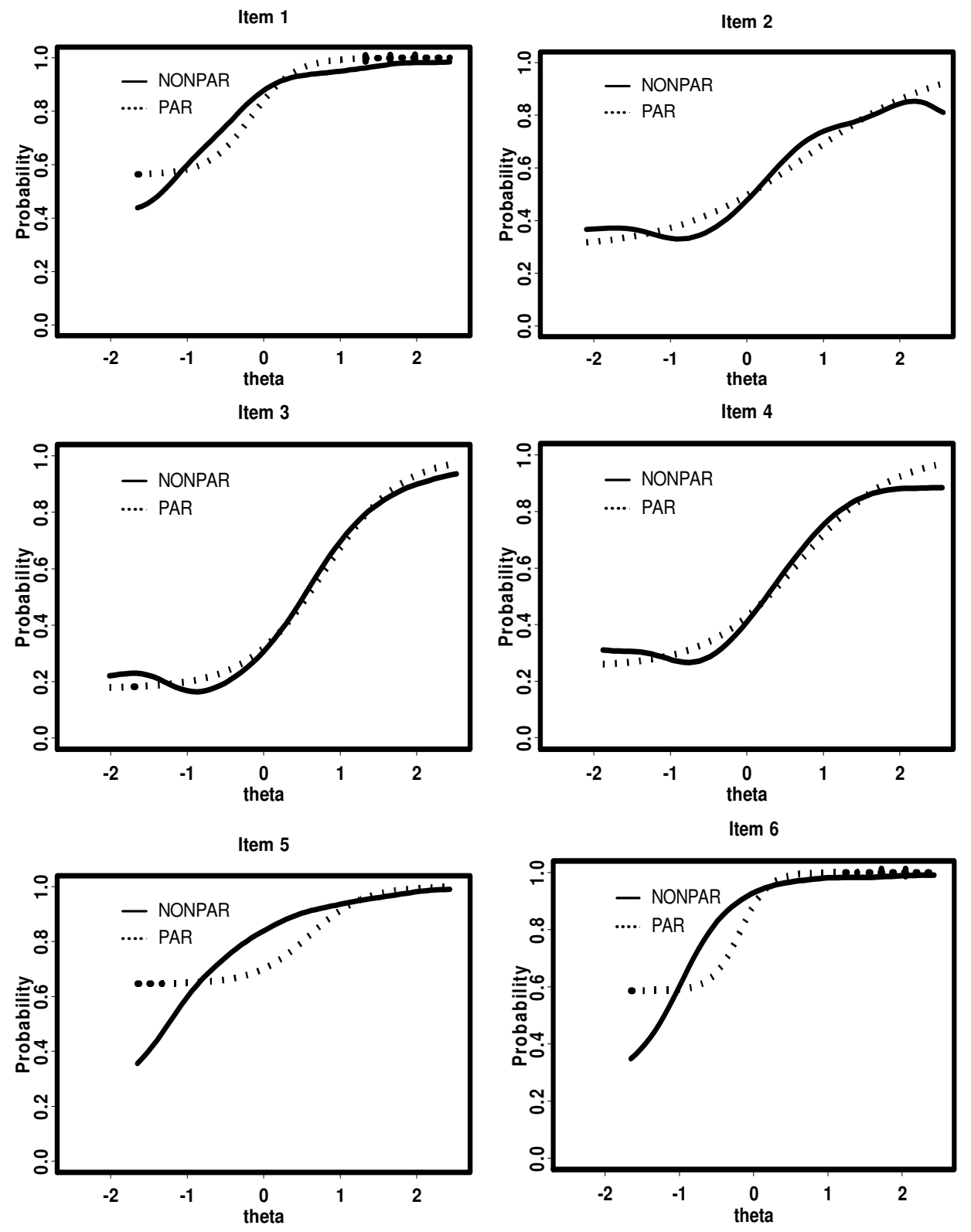

Item 7
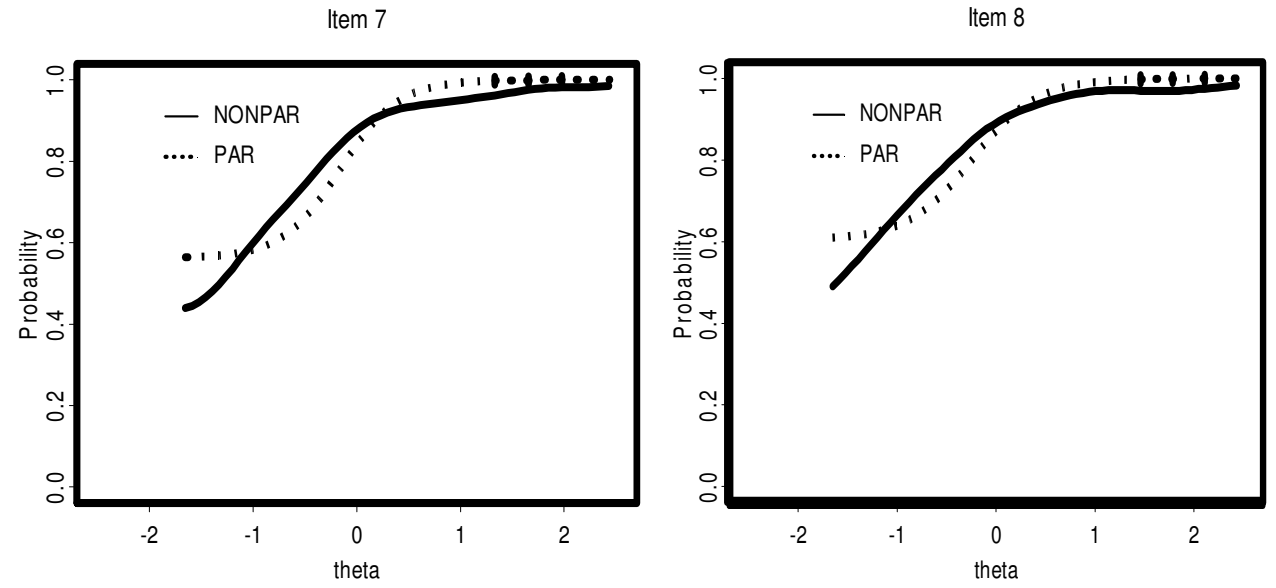
Item 9

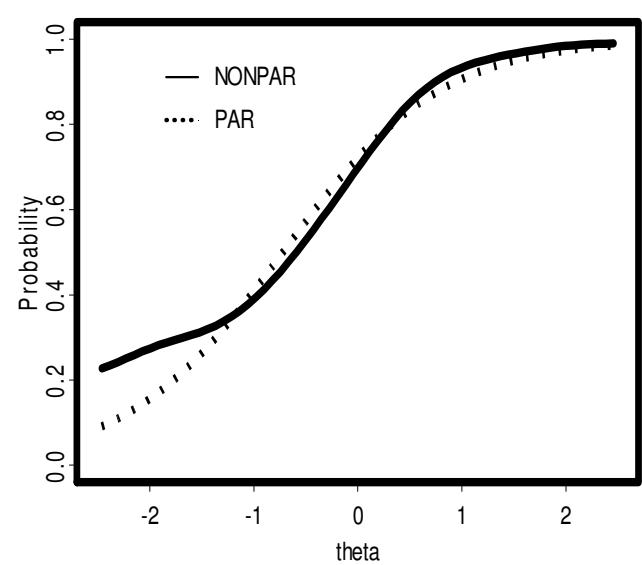

Item 11

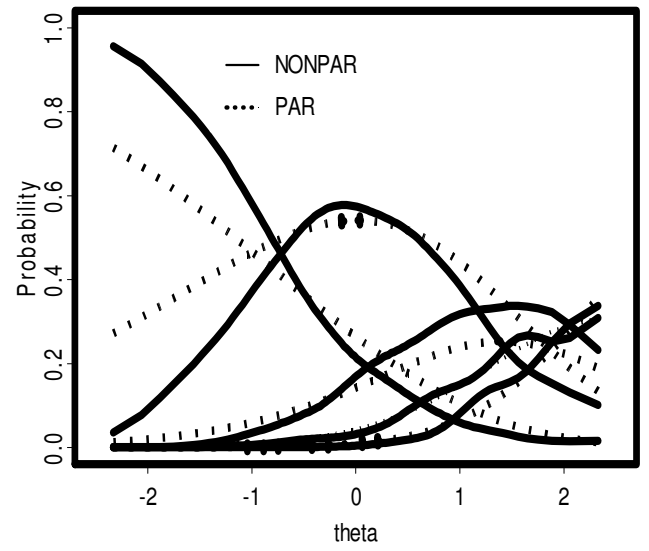

Item 10

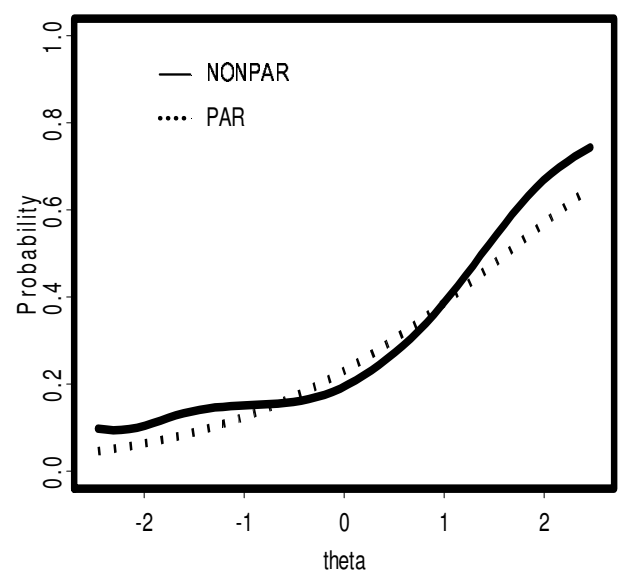

Item 12

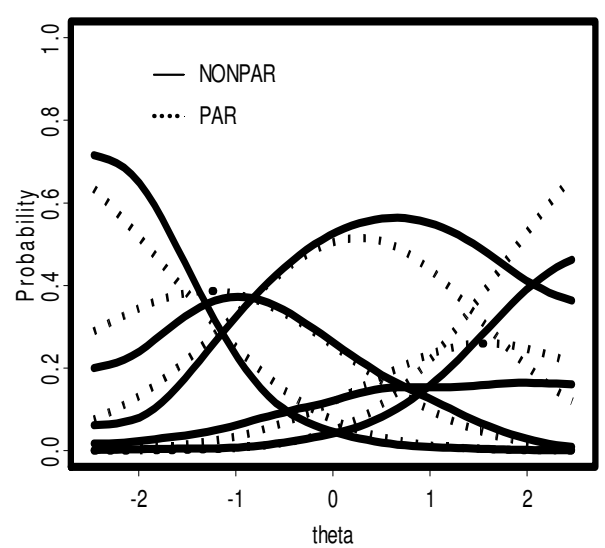




\section{CHAPTER 4}

\section{RESULTS}

\subsection{Study 1-Simulation Study}

\subsubsection{Statistical Properties}

Study 1 was a simulation study. In this study, the statistical properties of the three fit statistics were compared. Tables 4.1 through 4.12 summarize the Type I error rate and power for $\alpha=0.05$ and $\alpha=0.01$ under the thirty-six conditions from three fit statistics, RISE, $G^{2}$ and $S$ - $X^{2}$. Each table covered results for one ability distribution, one model combination, three sample sizes, and two test lengths.

Table 4.1 reports the empirical Type I error rates for RISE, $G^{2}$ and $S$ - $X^{2}$ based on normal ability distribution and 3PLM/2PLM/GPCM model combination. Table 4.2 reports results for 3PLM/2PLM/GRM model combination. Based on the two tables, the Type I error rate was controlled for RISE and highly deflated for $S$ - $X^{2}$ especially under 500- and 2,000-examinee conditions for each model. On the other hand, $G^{2}$ exhibited inflated Type I error rates. For the 3PLM, the Type I error rate was slightly inflated for the 500- and 2,000-examinee conditions, moderately inflated for the 10,000-examinee condition. For the 2PLM, $G^{2}$ was slightly inflated for the 500- and 2,000-examinee conditions; it was highly inflated for the 10,000-examinee conditions. For the GPCM and GRM, $G^{2}$ was slightly inflated for the 500-examinee condition, moderately inflated for the 2,000-examinee condition and highly inflated for the10,000-examinee condition.

In summary, RISE performed satisfactorily in that the Type I error rates were not inflated or affected by sample size and test length. $S-X^{2}$ exhibited highly deflated Type I error rates; however, as sample size increased and test length decreased, the Type I error rates increased toward the nominal alpha level. $G^{2}$ did not control Type I error 
rates for most conditions and Type I error rates increased while sample size increased; however, test length did not have any impact on it.

Tables 4.3 and 4.4 report the empirical power rates for RISE, $G^{2}$ and $S$ - $X^{2}$ across the conditions for the two model combinations in the mixed format test respectively. Because power is conditional on controlling the Type I error rate at the nominal alpha level ( $\alpha=0.05$ and $\alpha=0.01$ ), the power rates corresponding to the Type I error rates greater than 0.07 were $\mathrm{X}$-ed out for $\alpha=0.05$ and those greater than 0.03 were $\mathrm{X}$-ed out for $\alpha=0.01$. It was not appropriate to compare power rates between the three fit statistics for those $\mathrm{X}$-ed out cases.

In summary, as sample size increased, all three fit statistics exhibited increased power for each model. RISE exhibited more power than the valid power values of $G^{2}$. On the other hand, compared to $S-X^{2}, R I S E$ exhibited more power to detect misfit under 500- and 2,000-examinee conditions for each model.

Table 4.5 reports the empirical Type I error rates for RISE, $G^{2}$ and $S-X^{2}$ based on negatively-skewed ability distribution and 3PLM/2PLM/GPCM model combination. Table 4.6 reports results for 3PLM/2PLM/GRM model combination. The two tables indicated that the Type I error rate across all conditions was controlled for RISE only. $G^{2}$ exhibited inflated Type I error rates. For the 3PLM, the Type I error rate was slightly inflated for the 500- and 2,000-examinee conditions and it was moderately inflated for the 10,000-examinee condition. For the $2 \mathrm{PLM}, G^{2}$ was slightly inflated for the 500 - and 2,000-examinee conditions; it was highly inflated for the 10,000-examinee conditions. For the GPCM and GRM, $G^{2}$ was slightly inflated for the 500-examinee condition, moderately inflated for the 2,000-examinee condition and highly inflated for the10,000-examinee condition. $S-X^{2}$ only controlled Type I error rates for 500-examinee and 60-item condition for each model. It exhibited inflated Type I error rates for all 
other conditions. For the 3PLM, the Type I error rate was slightly inflated for the 500-examinee with 30-item and 2,000-examinee with 60-item conditions; it was highly inflated for other conditions. For the 2PLM, $S$ - $X^{2}$ was moderately inflated for the 500-examinee with 30-item and 2,000-examinee with 60-item conditions, highly inflated for other conditions. For the GPCM and GRM, $S$ - $X^{2}$ was highly inflated for all conditions.

In summary, under the negatively-skewed ability distribution, RISE also outperformed the other two fit statistics in that the Type I error rates were not inflated and not affected by sample size and test length. $G^{2}$ did not control Type I error rates and showed increasingly inflated Type I error rates as sample size increased. $S$ - $X^{2}$ did not control Type I error rates and exhibited a clear pattern that Type I error rates increased while sample size increased and test length decreased.

Tables 4.7 and 4.8 report the empirical power rates for RISE, $G^{2}$ and $S-X^{2}$ across the conditions for the two model combinations in the mixed format test respectively. Because power is conditional on controlling the Type I error rate at the nominal alpha level ( $\alpha=0.05$ and $\alpha=0.01$ ), the power rates corresponding to the Type I error rates greater than 0.07 were $\mathrm{X}$-ed out for $\alpha=0.05$ and those greater than 0.03 were $\mathrm{X}$-ed out for $\alpha=0.01$. It was not appropriate to compare power rates between $G^{2}, S$ - $X^{2}$ and $R I S E$ for those X-ed out cases.

In summary, as sample size increased, all three fit statistics exhibited increased power for each model. RISE exhibited much more power than the valid power values of $G^{2}$ and $S-X^{2}$

Table 4.9 reports the empirical Type I error rates for RISE, $G^{2}$ and $S-X^{2}$ based on positively-skewed ability distribution and 3PLM/2PLM/GPCM model combination. Table 4.10 reports results for 3PLM/2PLM/GRM model combination. The two tables 
demonstrated that the Type I error rate was controlled for RISE across all conditions. $G^{2}$ exhibited inflated Type I error rates. For the 3PLM and 2PLM, the Type I error rate was slightly inflated for the 500- and 2,000-examinee conditions, highly inflated for the 10,000-examinee condition. For the GPCM and GRM, $G^{2}$ was moderately inflated for the 500-examinee and the 2,000-examinee conditions and highly inflated for the10,000-examinee condition. $S-X^{2}$ only controlled Type I error rates for 500-examinee and 60-item condition for 3PLM and 2PLM. It exhibited inflated Type I error rates for all other conditions. For the 3PLM, the Type I error rate was moderately inflated for the 500-examinee with 30-item and 2,000-examinee with 60-item conditions, highly inflated for other conditions. For the 2PLM, $S$ - $X^{2}$ was moderately inflated for the 500-examinee with 30-item and 2,000-examinee with 60-item conditions, highly inflated for other conditions. For the GPCM and GRM, $S-X^{2}$ was highly inflated for all conditions.

In summary, under the positively-skewed ability distribution, RISE also outperformed the other two fit statistics in that the Type I error rates were not inflated and not affected by sample size and test length. $G^{2}$ did not control Type I error rates and showed increasingly inflated Type I error rates as sample size increased. $S$ - $X^{2} \operatorname{did}$ not control Type I error rates and had clear pattern that Type I error rates increased while sample size increased and test length decreased.

Tables 4.11 and 4.12 report the empirical power rates for RISE, $G^{2}$ and $S$ - $X^{2}$ across the conditions for the two model combinations in the mixed format test respectively. Because power is conditional on controlling the Type I error rate at the nominal alpha level ( $\alpha=0.05$ and $\alpha=0.01$ ), the power rates corresponding to the Type I error rates greater than 0.07 were $\mathrm{X}$-ed out for $\alpha=0.05$ and those greater than 0.03 were $\mathrm{X}$-ed out 
for $\alpha=0.01$. It was not appropriate to compare power rates between $G^{2}, S-X^{2}$ and $R I S E$ for those X-ed out cases.

In summary, as sample size increased, all three fit statistics exhibited increased power for each model. RISE exhibited much more power than the valid power values of $G^{2}$ and $S-X^{2}$

\subsubsection{Model Misfit Consequence}

\subsubsection{Score Distribution}

One of the simulated data sets (normal ability distribution, 10,000-examinee, 60-item and 3PLM/2PLM/GPCM) was used to address the model misfit consequence on score distribution. Figures 4.1 through 4.3 report the observed score distribution (blue solid line) and the expected score distribution (red dotted line) before and after replacement of misfitting items detected by three fit statistics. However, to illustrate the misfit consequence on practical use, three cuts $(40,60,80)$ were selected to quantify the difference in distributions attributed to the replacement of misfitting items. The difference in the percentage of examinees at each cut was $1 \%, 2 \%$ and $2 \%$ respectively. 0.005 was chosen to be a criterion, so the impact on score distribution before and after replacement was significant if the value in each cell was greater than 0.005 . The reason to choose 0.005 as the criterion was because it was average of differences at all score points. Table 4.13 reports the results. Based on both the table and the plots, there was no significant model misfit consequence on score distribution for the given simulated data. It is important to note that this result may have occurred because the simulated misfit did not have a consequential effect on test score distributions.

\subsubsection{TCC}

The same simulation data were used to address the model misfit consequence on TCC. Figure 4.4 reports the nonparametric TCC (blue solid line) and the parametric 
TCC (red dotted line) before and after replacement of misfitting items detected by RISE. However, to illustrate the misfit consequence on practical use, three cuts, $-1.41,0.33$ and1.11 were transformed from raw score scale to quantify the change of TCC difference. The cut of 0.5 was chosen because if the difference is larger than 0.5 , it would be rounded to 1.0. Table 4.14 reports the results. Based on the table and the plots, there was no significant model misfit consequence on TCC for the given simulated data at the cuts.

\subsection{Study 2-Empirical Study}

\subsubsection{Residual Plots on Misfitting Items}

Three fit statistics were applied to one empirical data which contained 32 multiple choice items fitted by 3PLM, 4 short answer items fitted by 2PLM and 6 constructed response items fitted by GPCM. Three sample sizes $(500,2,000$ and 10,000) were randomly drawn without replacement from a larger sample size. Table 4.15 summarized the number and percentage of misfitting items detected by each fit statistic at each sample size. The misfitting items were listed in the last column. At sample size 10,000, $R I S E$ and $S-X^{2}$ flagged very similar misfitting items while $G^{2}$ flagged almost all items as misfitting. Figure 4.5 through 4.12 show the residual plots on misfitting items detected by three fit statistics. Each misfitting polytomous item had one aggregated raw residual plot weighted by score categories.

\subsubsection{Model Misfit Consequence on Score Distribution}

Figure 4.13 through 4.15 report the observed score distribution (blue solid line) and the expected score distribution (red dotted line) before and after replacement of misfitting items detected by three fit statistics at three sample sizes. To further illustrate the misfit consequence quantitatively and practically, three cuts $(25,40,55)$ were selected to assess the change of distribution difference. The percentage of examinees at 
each cut was $1 \%, 2 \%$ and $4 \%$ respectively. Table 4.16 reports the results. Based on both the table and the plots, there was no meaningful model misfit consequence on score distribution for the given data at each sample size. In addition, the three fit statistics did not lead to any meaningful distribution difference before and after replacement. The finding was consistent with the examination of statistical properties in simulation study. 
Table 4.1 Empirical Type I Error Rate for RISE, PARSCALE's $G^{2}$ and IRTFIT's S- $X^{2}$ Statistic, Normal Ability Distribution, 3PLM/2PLM/GPCM Model Combination

\begin{tabular}{|c|c|c|c|c|c|c|c|c|}
\hline $\begin{array}{c}\text { Sample } \\
\text { Size } \\
\end{array}$ & $\begin{array}{c}\text { Test } \\
\text { Length }\end{array}$ & Model & $\begin{array}{c}\text { RISE } \\
(0.05)\end{array}$ & $\begin{array}{c}G^{2} \\
(0.05)\end{array}$ & $\begin{array}{c}S-X^{2} \\
(0.05)\end{array}$ & $\begin{array}{c}\text { RISE } \\
(0.01)\end{array}$ & $\begin{array}{c}G^{2} \\
(0.01)\end{array}$ & $\begin{array}{c}S-X^{2} \\
(0.01)\end{array}$ \\
\hline \multirow[t]{6}{*}{500} & \multirow[t]{3}{*}{30} & 3PLM & 0.038 & 0.069 & 0.027 & 0.009 & 0.026 & 0.007 \\
\hline & & 2PLM & 0.041 & 0.085 & 0.005 & 0.011 & 0.045 & 0.000 \\
\hline & & GPCM & 0.050 & 0.060 & 0.008 & 0.012 & 0.008 & 0.000 \\
\hline & \multirow[t]{3}{*}{60} & 3PLM & 0.036 & 0.070 & 0.008 & 0.007 & 0.031 & 0.003 \\
\hline & & 2PLM & 0.045 & 0.070 & 0.003 & 0.009 & 0.023 & 0.001 \\
\hline & & GPCM & 0.052 & 0.079 & 0.001 & 0.010 & 0.021 & 0.000 \\
\hline \multirow[t]{6}{*}{2,000} & \multirow[t]{3}{*}{30} & 3PLM & 0.029 & 0.073 & 0.036 & 0.000 & 0.016 & 0.008 \\
\hline & & 2PLM & 0.045 & 0.085 & 0.018 & 0.006 & 0.013 & 0.003 \\
\hline & & GPCM & 0.042 & 0.225 & 0.005 & 0.001 & 0.070 & 0.000 \\
\hline & \multirow[t]{3}{*}{60} & 3PLM & 0.034 & 0.061 & 0.018 & 0.008 & 0.019 & 0.006 \\
\hline & & 2PLM & 0.049 & 0.074 & 0.010 & 0.013 & 0.011 & 0.001 \\
\hline & & GPCM & 0.032 & 0.119 & 0.001 & 0.011 & 0.029 & 0.000 \\
\hline \multirow[t]{6}{*}{10,000} & \multirow[t]{3}{*}{30} & 3PLM & 0.040 & 0.251 & 0.040 & 0.004 & 0.095 & 0.006 \\
\hline & & 2PLM & 0.039 & 0.538 & 0.055 & 0.002 & 0.283 & 0.008 \\
\hline & & GPCM & 0.053 & 0.995 & 0.063 & 0.012 & 0.995 & 0.013 \\
\hline & \multirow[t]{3}{*}{60} & 3PLM & 0.036 & 0.321 & 0.032 & 0.000 & 0.139 & 0.005 \\
\hline & & 2PLM & 0.042 & 0.466 & 0.031 & 0.004 & 0.230 & 0.004 \\
\hline & & GPCM & 0.048 & 0.931 & 0.026 & 0.005 & 0.843 & 0.008 \\
\hline
\end{tabular}


Table 4.2 Empirical Type I Error Rate for RISE, PARSCALE's $G^{2}$ and IRTFIT'S S- $X^{2}$ Statistic, Normal Ability Distribution, 3PLM/2PLM/GRM Model Combination

\begin{tabular}{|c|c|c|c|c|c|c|c|c|}
\hline $\begin{array}{c}\text { Sample } \\
\text { Size }\end{array}$ & $\begin{array}{c}\text { Test } \\
\text { Length }\end{array}$ & Model & $\begin{array}{l}\text { RISE } \\
(0.05)\end{array}$ & $\begin{array}{c}G^{2} \\
(0.05) \\
\end{array}$ & $\begin{array}{c}S-X^{2} \\
(0.05)\end{array}$ & $\begin{array}{l}\text { RISE } \\
(0.01) \\
\end{array}$ & $\begin{array}{c}G^{2} \\
(0.01) \\
\end{array}$ & $\begin{array}{c}S-X^{2} \\
(0.01) \\
\end{array}$ \\
\hline \multirow[t]{6}{*}{500} & \multirow[t]{3}{*}{30} & 3PLM & 0.031 & 0.063 & 0.022 & 0.011 & 0.016 & 0.008 \\
\hline & & 2PLM & 0.041 & 0.065 & 0.010 & 0.006 & 0.040 & 0.004 \\
\hline & & GRM & 0.032 & 0.078 & 0.009 & 0.010 & 0.018 & 0.001 \\
\hline & \multirow[t]{3}{*}{60} & 3PLM & 0.043 & 0.083 & 0.013 & 0.005 & 0.038 & 0.002 \\
\hline & & 2PLM & 0.045 & 0.085 & 0.003 & 0.008 & 0.038 & 0.000 \\
\hline & & GRM & 0.036 & 0.064 & 0.001 & 0.007 & 0.013 & 0.000 \\
\hline \multirow[t]{6}{*}{2,000} & \multirow[t]{3}{*}{30} & 3PLM & 0.041 & 0.076 & 0.037 & 0.000 & 0.021 & 0.011 \\
\hline & & 2PLM & 0.047 & 0.063 & 0.025 & 0.012 & 0.010 & 0.009 \\
\hline & & GRM & 0.040 & 0.420 & 0.016 & 0.009 & 0.185 & 0.005 \\
\hline & \multirow[t]{3}{*}{60} & 3PLM & 0.039 & 0.064 & 0.018 & 0.007 & 0.016 & 0.006 \\
\hline & & 2PLM & 0.050 & 0.073 & 0.011 & 0.010 & 0.009 & 0.002 \\
\hline & & GRM & 0.032 & 0.398 & 0.007 & 0.000 & 0.176 & 0.000 \\
\hline \multirow[t]{6}{*}{10,000} & \multirow[t]{3}{*}{30} & 3PLM & 0.048 & 0.484 & 0.043 & 0.004 & 0.241 & 0.013 \\
\hline & & 2PLM & 0.046 & 0.843 & 0.051 & 0.002 & 0.618 & 0.010 \\
\hline & & GRM & 0.053 & 1.000 & 0.064 & 0.005 & 1.000 & 0.008 \\
\hline & \multirow[t]{3}{*}{60} & 3PLM & 0.042 & 0.293 & 0.039 & 0.006 & 0.114 & 0.007 \\
\hline & & 2PLM & 0.052 & 0.489 & 0.040 & 0.001 & 0.223 & 0.004 \\
\hline & & GRM & 0.044 & 1.000 & 0.050 & 0.005 & 1.000 & 0.001 \\
\hline
\end{tabular}


Table 4.3 Empirical Detection Rate for RISE, PARSCALE's $G^{2}$ and IRTFIT'S S- $X^{2}$ Statistic, Normal Ability Distribution, 3PLM/2PLM/GPCM Model Combination

\begin{tabular}{|c|c|c|c|c|c|c|c|c|}
\hline $\begin{array}{c}\text { Sample } \\
\text { Size } \\
\end{array}$ & $\begin{array}{c}\text { Test } \\
\text { Length }\end{array}$ & Model & $\begin{array}{c}\text { RISE } \\
(0.05)\end{array}$ & $\begin{array}{c}G^{2} \\
(0.05) \\
\end{array}$ & $\begin{array}{c}S-X^{2} \\
(0.05)\end{array}$ & $\begin{array}{c}\text { RISE } \\
(0.01)\end{array}$ & $\begin{array}{c}G^{2} \\
(0.01)\end{array}$ & $\begin{array}{c}S-X^{2} \\
(0.01)\end{array}$ \\
\hline \multirow[t]{6}{*}{500} & \multirow[t]{3}{*}{30} & 3PLM & 0.298 & 0.153 & 0.035 & 0.145 & 0.028 & 0.010 \\
\hline & & 2PLM & 0.270 & 0.140 & 0.070 & 0.129 & D.OAO & 0.010 \\
\hline & & GPCM & 0.341 & 0.130 & 0.150 & 0.172 & 0.010 & 0.080 \\
\hline & \multirow[t]{3}{*}{60} & 3PLM & 0.369 & 0.489 & 0.021 & 0.200 & 0.049 & 0.006 \\
\hline & & 2PLM & 0.310 & 0.095 & 0.010 & 0.178 & 0.015 & 0.005 \\
\hline & & GPCM & 0.289 & $Q 160$ & 0.050 & 0.103 & 0.045 & 0.020 \\
\hline \multirow[t]{6}{*}{2,000} & \multirow[t]{3}{*}{30} & 3PLM & 0.596 & U. & 0.253 & 0.371 & 0.138 & 0.103 \\
\hline & & 2PLM & 0.892 & 0.290 & 0.400 & 0.406 & 0.070 & 0.180 \\
\hline & & GPCM & 0.910 & & 0.830 & 0.790 & $Q 560$ & 0.680 \\
\hline & \multirow[t]{3}{*}{60} & 3PLM & 0.612 & 0.636 & 0.053 & 0.322 & 0.419 & 0.093 \\
\hline & & 2PLM & 0.785 & 0.330 & 0.185 & 0.555 & 0.135 & 0.085 \\
\hline & & GPCM & 0.873 & 0685 & 0.445 & 0.626 & 0.440 & 0.275 \\
\hline \multirow[t]{6}{*}{10,000} & \multirow[t]{3}{*}{30} & 3PLM & 0.999 & & 0.985 & 0.999 & 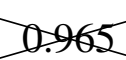 & 0.941 \\
\hline & & 2PLM & 1.000 & 100 & 1.000 & 1.000 & 0.980 & 1.000 \\
\hline & & GPCM & 1.000 & 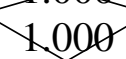 & 1.000 & 1.000 & & 1.000 \\
\hline & \multirow[t]{3}{*}{60} & 3PLM & 0.998 & & 0.969 & 0.960 & & 0.938 \\
\hline & & 2PLM & 1.000 & 1000 & 1.000 & 1.000 & & 0.980 \\
\hline & & GPCM & 1.000 & 1.000 & 1.000 & 1.000 & 1.000 & 1.000 \\
\hline
\end{tabular}

X-ed out cells represent conditions in which the test statistic exhibited inflated Type I error rates. 
Table 4.4 Empirical Detection Rate for RISE, PARSCALE's $G^{2}$ and IRTFIT'S S- $X^{2}$ Statistic, Normal Ability Distribution, 3PLM/2PLM/GRM Model Combination

\begin{tabular}{|c|c|c|c|c|c|c|c|c|}
\hline $\begin{array}{c}\text { Sample } \\
\text { Size } \\
\end{array}$ & $\begin{array}{c}\text { Test } \\
\text { Length }\end{array}$ & Model & $\begin{array}{c}\text { RISE } \\
(0.05)\end{array}$ & $\begin{array}{c}G^{2} \\
(0.05) \\
\end{array}$ & $\begin{array}{c}S-X^{2} \\
(0.05)\end{array}$ & $\begin{array}{c}\text { RISE } \\
(0.01)\end{array}$ & $\begin{array}{c}G^{2} \\
(0.01)\end{array}$ & $\begin{array}{c}S-X^{2} \\
(0.01)\end{array}$ \\
\hline \multirow[t]{6}{*}{500} & \multirow[t]{3}{*}{30} & 3PLM & 0.303 & 0.065 & 0.029 & 0.188 & 0.028 & 0.020 \\
\hline & & 2PLM & 0.277 & 0.120 & 0.066 & 0.220 & 0.020 & 0.030 \\
\hline & & GRM & 0.346 & Q110 & 0.143 & 0.272 & 0.010 & 0.100 \\
\hline & \multirow[t]{3}{*}{60} & 3PLM & 0.374 & & 0.022 & 0.240 & & 0.003 \\
\hline & & 2PLM & 0.337 & 0.080 & 0.011 & 0.208 & 0.025 & 0.007 \\
\hline & & GRM & 0.299 & 0.075 & 0.080 & 0.203 & 0.010 & 0.025 \\
\hline \multirow[t]{6}{*}{2,000} & \multirow[t]{3}{*}{30} & 3PLM & 0.636 & O. & 0.257 & 0.471 & 0.063 & 0.106 \\
\hline & & 2PLM & 0.899 & 0.240 & 0.523 & 0.506 & 0.080 & 0.189 \\
\hline & & GRM & 0.901 & & 0.801 & 0.799 & 528 & 0.643 \\
\hline & \multirow[t]{3}{*}{60} & 3PLM & 0.598 & 0.460 & 0.153 & 0.330 & 0.236 & 0.093 \\
\hline & & 2PLM & 0.858 & 0.245 & 0.295 & 0.505 & 0.060 & 0.185 \\
\hline & & GRM & 0.871 & 0575 & 0.445 & 0.626 & & 0.294 \\
\hline \multirow[t]{6}{*}{10,000} & \multirow[t]{3}{*}{30} & 3PLM & 0.996 & & 0.980 & 0.990 & & 0.932 \\
\hline & & 2PLM & 1.000 & 1000 & 1.000 & 1.000 & 0.99 & 1.000 \\
\hline & & GRM & 1.000 & & 1.000 & 1.000 & & 1.000 \\
\hline & \multirow[t]{3}{*}{60} & 3PLM & 0.998 & & 0.969 & 0.960 & & 0.938 \\
\hline & & 2PLM & 1.000 & 1.06 & 1.000 & 1.000 & & 1.000 \\
\hline & & GRM & 1.000 & 1.000 & 0.986 & 0.934 & 1.000 & 0.934 \\
\hline
\end{tabular}

X-ed out cells represent conditions in which the test statistic exhibited inflated Type I error rates. 
Table 4.5 Empirical Type I Error Rate for RISE, PARSCALE's $G^{2}$ and IRTFIT's $S$ - $X^{2}$ Statistic, Negatively-Skewed Ability Distribution, 3PLM/2PLM/GPCM Model Combination

\begin{tabular}{|c|c|c|c|c|c|c|c|c|}
\hline $\begin{array}{c}\text { Sample } \\
\text { Size }\end{array}$ & $\begin{array}{c}\text { Test } \\
\text { Length }\end{array}$ & Model & $\begin{array}{l}\text { RISE } \\
(0.05)\end{array}$ & $\begin{array}{c}G^{2} \\
(0.05)\end{array}$ & $\begin{array}{c}S-X^{2} \\
(0.05)\end{array}$ & $\begin{array}{l}\text { RISE } \\
(0.01)\end{array}$ & $\begin{array}{c}G^{2} \\
(0.01)\end{array}$ & $\begin{array}{c}S-X^{2} \\
(0.01)\end{array}$ \\
\hline \multirow[t]{6}{*}{500} & \multirow[t]{3}{*}{30} & 3PLM & 0.029 & 0.070 & 0.194 & 0.000 & 0.017 & 0.057 \\
\hline & & 2PLM & 0.031 & 0.073 & 0.273 & 0.011 & 0.005 & 0.100 \\
\hline & & GPCM & 0.038 & 0.100 & 0.980 & 0.010 & 0.040 & 0.870 \\
\hline & \multirow[t]{3}{*}{60} & 3PLM & 0.033 & 0.082 & 0.031 & 0.008 & 0.020 & 0.005 \\
\hline & & 2PLM & 0.045 & 0.114 & 0.015 & 0.009 & 0.020 & 0.003 \\
\hline & & GPCM & 0.030 & 0.168 & 0.035 & 0.007 & 0.068 & 0.014 \\
\hline \multirow[t]{6}{*}{2,000} & \multirow[t]{3}{*}{30} & 3PLM & 0.040 & 0.126 & 0.807 & 0.001 & 0.038 & 0.654 \\
\hline & & 2PLM & 0.041 & 0.143 & 0.988 & 0.000 & 0.043 & 0.908 \\
\hline & & GPCM & 0.047 & 0.638 & 1.000 & 0.004 & 0.423 & 1.000 \\
\hline & \multirow[t]{3}{*}{60} & 3PLM & 0.035 & 0.093 & 0.284 & 0.003 & 0.030 & 0.137 \\
\hline & & 2PLM & 0.049 & 0.108 & 0.460 & 0.010 & 0.016 & 0.361 \\
\hline & & GPCM & 0.038 & 0.403 & 0.981 & 0.002 & 0.213 & 0.891 \\
\hline \multirow[t]{6}{*}{10,000} & \multirow[t]{3}{*}{30} & 3PLM & 0.046 & 0.584 & 0.995 & 0.005 & 0.393 & 0.986 \\
\hline & & 2PLM & 0.042 & 0.855 & 1.000 & 0.011 & 0.638 & 1.000 \\
\hline & & GPCM & 0.050 & 1.000 & 1.000 & 0.012 & 1.000 & 1.000 \\
\hline & \multirow[t]{3}{*}{60} & 3PLM & 0.041 & 0.309 & 0.943 & 0.006 & 0.157 & 0.866 \\
\hline & & 2PLM & 0.053 & 0.619 & 1.000 & 0.010 & 0.365 & 1.000 \\
\hline & & GPCM & 0.044 & 0.990 & 1.000 & 0.005 & 0.974 & 1.000 \\
\hline
\end{tabular}


Table 4.6 Empirical Type I Error Rate for RISE, PARSCALE's $G^{2}$ and IRTFIT's $S$ - $X^{2}$ Statistic, Negatively-Skewed Ability Distribution, 3PLM/2PLM/GRM Model Combination

\begin{tabular}{|c|c|c|c|c|c|c|c|c|}
\hline $\begin{array}{c}\text { Sample } \\
\text { Size }\end{array}$ & $\begin{array}{c}\text { Test } \\
\text { Length }\end{array}$ & Model & $\begin{array}{l}\text { RISE } \\
(0.05)\end{array}$ & $\begin{array}{c}G^{2} \\
(0.05)\end{array}$ & $\begin{array}{c}S-X^{2} \\
(0.05)\end{array}$ & $\begin{array}{l}\text { RISE } \\
(0.01)\end{array}$ & $\begin{array}{c}G^{2} \\
(0.01)\end{array}$ & $\begin{array}{c}S-X^{2} \\
(0.01)\end{array}$ \\
\hline \multirow[t]{6}{*}{500} & \multirow[t]{3}{*}{30} & 3PLM & 0.043 & 0.071 & 0.201 & 0.008 & 0.011 & 0.101 \\
\hline & & 2PLM & 0.039 & 0.075 & 0.236 & 0.012 & 0.018 & 0.115 \\
\hline & & GRM & 0.042 & 0.090 & 0.889 & 0.005 & 0.010 & 0.745 \\
\hline & \multirow[t]{3}{*}{60} & 3PLM & 0.030 & 0.074 & 0.042 & 0.007 & 0.018 & 0.012 \\
\hline & & 2PLM & 0.047 & 0.074 & 0.027 & 0.001 & 0.021 & 0.014 \\
\hline & & GRM & 0.026 & 0.103 & 0.045 & 0.004 & 0.025 & 0.010 \\
\hline \multirow[t]{6}{*}{2,000} & \multirow[t]{3}{*}{30} & 3PLM & 0.035 & 0.152 & 0.798 & 0.000 & 0.061 & 0.545 \\
\hline & & 2PLM & 0.049 & 0.180 & 0.899 & 0.000 & 0.063 & 0.677 \\
\hline & & GRM & 0.047 & 0.420 & 0.936 & 0.010 & 0.180 & 0.821 \\
\hline & \multirow[t]{3}{*}{60} & 3PLM & 0.046 & 0.096 & 0.275 & 0.006 & 0.033 & 0.121 \\
\hline & & 2PLM & 0.051 & 0.106 & 0.303 & 0.005 & 0.031 & 0.190 \\
\hline & & GRM & 0.048 & 0.331 & 0.875 & 0.002 & 0.123 & 0.744 \\
\hline \multirow[t]{6}{*}{10,000} & \multirow[t]{3}{*}{30} & 3PLM & 0.029 & 0.789 & 0.991 & 0.004 & 0.633 & 0.936 \\
\hline & & 2PLM & 0.042 & 0.990 & 0.990 & 0.013 & 0.960 & 0.990 \\
\hline & & GRM & 0.053 & 1.000 & 0.998 & 0.012 & 1.000 & 0.975 \\
\hline & \multirow[t]{3}{*}{60} & 3PLM & 0.035 & 0.377 & 0.967 & 0.007 & 0.197 & 0.860 \\
\hline & & 2PLM & 0.050 & 0.748 & 0.987 & 0.009 & 0.454 & 0.900 \\
\hline & & GRM & 0.047 & 1.000 & 0.997 & 0.006 & 1.000 & 0.997 \\
\hline
\end{tabular}


Table 4.7 Empirical Detection Rate for RISE, PARSCALE's $G^{2}$ and IRTFIT's $S$ - $X^{2}$ Statistic, Negatively-Skewed Ability Distribution, 3PLM/2PLM/GPCM Model Combination

\begin{tabular}{|c|c|c|c|c|c|c|c|c|}
\hline $\begin{array}{c}\text { Sample } \\
\text { Size }\end{array}$ & $\begin{array}{c}\text { Test } \\
\text { Length }\end{array}$ & Model & $\begin{array}{l}\text { RISE } \\
(0.05)\end{array}$ & $\begin{array}{c}G^{2} \\
(0.05)\end{array}$ & $\begin{array}{c}S-X^{2} \\
(0.05)\end{array}$ & $\begin{array}{c}\text { RISE } \\
(0.01)\end{array}$ & $\begin{array}{c}G^{2} \\
(0.01)\end{array}$ & $\begin{array}{c}S-X^{2} \\
(0.01)\end{array}$ \\
\hline \multirow[t]{6}{*}{500} & \multirow[t]{3}{*}{30} & 3PLM & 0.410 & 0.190 & 0.235 & 0.258 & 0.118 & 0.08 \\
\hline & & 2PLM & 0.367 & 0.050 & 0.200 & 0.230 & 0.000 & 0.08 \\
\hline & & GPCM & 0.446 & & & 0.372 & & \\
\hline & \multirow[t]{3}{*}{60} & 3PLM & 0.394 & & 0.173 & 0.390 & 0 & 0.099 \\
\hline & & 2PLM & 0.327 & 2.195 & 0.060 & 0.308 & 0.065 & 0.085 \\
\hline & & GPCM & 0.289 & 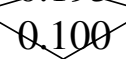 & 0.055 & 0.203 & Q.05 & 0.030 \\
\hline \multirow[t]{6}{*}{2,000} & \multirow[t]{3}{*}{30} & 3PLM & 0.696 & & & 0.571 & & \\
\hline & & 2PLM & 0.899 & 10 & 0.970 & 0.508 & 72 & .870 \\
\hline & & GPCM & 0.903 & & & 0.801 & & \\
\hline & \multirow[t]{3}{*}{60} & 3PLM & 0.798 & & & 0.730 & 0.516 & \\
\hline & & 2PLM & 0.888 & 1.225 & 0.330 & 0.624 & 0.080 & 0.225 \\
\hline & & GPCM & 0.801 & & & 0.613 & & \\
\hline \multirow[t]{6}{*}{10,000} & \multirow[t]{3}{*}{30} & 3PLM & 0.987 & & & 0.900 & & \\
\hline & & 2PLM & 0.934 & Defo & 100 & 0.911 & 50 & $\theta+0$ \\
\hline & & GPCM & 1.000 & & & 1.000 & & \\
\hline & \multirow[t]{3}{*}{60} & 3PLM & 0.998 & & & 0.960 & & \\
\hline & & 2PLM & 0.867 & & 0.585 & 0.802 & & 0.520 \\
\hline & & GPCM & 1.000 & 1.000 & 1000 & 0.925 & 1.000 & D.000 \\
\hline
\end{tabular}

X-ed out cells represent conditions in which the test statistic exhibited inflated Type I error rates. 
Table 4.8 Empirical Detection Rate for RISE, PARSCALE's $G^{2}$ and IRTFIT's $S$ - $X^{2}$ Statistic, Negatively-Skewed Ability Distribution, 3PLM/2PLM/GRM Model Combination

\begin{tabular}{|c|c|c|c|c|c|c|c|c|}
\hline $\begin{array}{c}\text { Sample } \\
\text { Size }\end{array}$ & $\begin{array}{c}\text { Test } \\
\text { Length }\end{array}$ & Model & $\begin{array}{l}\text { RISE } \\
(0.05)\end{array}$ & $\begin{array}{c}G^{2} \\
(0.05)\end{array}$ & $\begin{array}{c}S-X^{2} \\
(0.05)\end{array}$ & $\begin{array}{c}\text { RISE } \\
(0.01)\end{array}$ & $\begin{array}{c}G^{2} \\
(0.01)\end{array}$ & $\begin{array}{c}S-X^{2} \\
(0.01)\end{array}$ \\
\hline \multirow[t]{6}{*}{500} & \multirow[t]{3}{*}{30} & 3PLM & 0.387 & 0.080 & 0.21 & 0.385 & 0.043 & 0.18 \\
\hline & & 2PLM & 0.567 & 0.110 & 0.200 & 0.303 & 0.020 & 4 \\
\hline & & GRM & 0.546 & & & 0.327 & 0.030 & \\
\hline & \multirow[t]{3}{*}{60} & 3PLM & 0.574 & 0.254 & 0.144 & 0.409 & 0.200 & 0.109 \\
\hline & & 2PLM & 0.627 & 0.155 & 0.105 & 0.391 & 0.065 & 0.085 \\
\hline & & GRM & 0.689 & 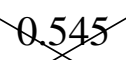 & 0.090 & 0.602 & 0.505 & 0.060 \\
\hline \multirow[t]{6}{*}{2,000} & \multirow[t]{3}{*}{30} & 3PLM & 0.769 & & & 0.517 & & \\
\hline & & 2PLM & 0.899 & & 0.85 & 0.680 & W. & 0.86 \\
\hline & & GRM & 0.924 & & & 0.801 & & \\
\hline & \multirow[t]{3}{*}{60} & 3PLM & 0.888 & & & 0.703 & 0.339 & \\
\hline & & 2PLM & 0.765 & 280 & 0.560 & 0.602 & 0.140 & 0.25 \\
\hline & & GRM & 0.910 & & & 0.747 & & \\
\hline \multirow[t]{6}{*}{10,000} & \multirow[t]{3}{*}{30} & 3PLM & 0.991 & & & 0.847 & & \\
\hline & & 2PLM & 0.920 & & Dog & 0.811 & Ase2n & 0.898 \\
\hline & & GRM & 0.998 & & & 0.998 & & \\
\hline & \multirow[t]{3}{*}{60} & 3PLM & 0.998 & & & 0.940 & & \\
\hline & & 2PLM & 0.864 & & 0.88 & 0.720 & & 0.5 \\
\hline & & GRM & 1.000 & I. & 0.911 & 0.931 & 1.000 & 0.904 \\
\hline
\end{tabular}

X-ed out cells represent conditions in which the test statistic exhibited inflated Type I error rates. 
Table 4.9 Empirical Type I Error Rate for RISE, PARSCALE's $G^{2}$ and IRTFIT's $S$ - $X^{2}$ Statistic, Positively-Skewed Ability Distribution, 3PLM/2PLM/GPCM Model Combination

\begin{tabular}{|c|c|c|c|c|c|c|c|c|}
\hline $\begin{array}{c}\text { Sample } \\
\text { Size }\end{array}$ & $\begin{array}{c}\text { Test } \\
\text { Length }\end{array}$ & Model & $\begin{array}{l}\text { RISE } \\
(0.05)\end{array}$ & $\begin{array}{c}G^{2} \\
(0.05)\end{array}$ & $\begin{array}{c}S-X^{2} \\
(0.05)\end{array}$ & $\begin{array}{l}\text { RISE } \\
(0.01)\end{array}$ & $\begin{array}{c}G^{2} \\
(0.01)\end{array}$ & $\begin{array}{c}S-X^{2} \\
(0.01)\end{array}$ \\
\hline \multirow[t]{6}{*}{500} & \multirow[t]{3}{*}{30} & 3PLM & 0.034 & 0.072 & 0.255 & 0.013 & 0.291 & 0.105 \\
\hline & & 2PLM & 0.042 & 0.086 & 0.360 & 0.015 & 0.248 & 0.158 \\
\hline & & GPCM & 0.041 & 0.095 & 0.830 & 0.007 & 0.038 & 0.673 \\
\hline & \multirow[t]{3}{*}{60} & 3PLM & 0.039 & 0.278 & 0.041 & 0.008 & 0.218 & 0.014 \\
\hline & & 2PLM & 0.045 & 0.179 & 0.027 & 0.007 & 0.188 & 0.005 \\
\hline & & GPCM & 0.048 & 0.369 & 0.211 & 0.001 & 0.210 & 0.090 \\
\hline \multirow[t]{6}{*}{2,000} & \multirow[t]{3}{*}{30} & 3PLM & 0.029 & 0.089 & 0.955 & 0.000 & 0.016 & 0.868 \\
\hline & & 2PLM & 0.032 & 0.080 & 0.963 & 0.009 & 0.015 & 0.903 \\
\hline & & GPCM & 0.028 & 0.480 & 0.990 & 0.011 & 0.298 & 0.990 \\
\hline & \multirow[t]{3}{*}{60} & 3PLM & 0.033 & 0.093 & 0.393 & 0.003 & 0.025 & 0.184 \\
\hline & & 2PLM & 0.040 & 0.125 & 0.530 & 0.004 & 0.043 & 0.281 \\
\hline & & GPCM & 0.037 & 0.454 & 0.884 & 0.002 & 0.330 & 0.790 \\
\hline \multirow[t]{6}{*}{10,000} & \multirow[t]{3}{*}{30} & 3PLM & 0.050 & 0.653 & 0.990 & 0.006 & 0.434 & 0.990 \\
\hline & & 2PLM & 0.045 & 0.803 & 0.990 & 0.002 & 0.743 & 0.990 \\
\hline & & GPCM & 0.045 & 1.000 & 0.990 & 0.012 & 0.998 & 0.990 \\
\hline & \multirow[t]{3}{*}{60} & 3PLM & 0.051 & 0.632 & 0.988 & 0.010 & 0.445 & 0.973 \\
\hline & & 2PLM & 0.053 & 0.750 & 0.990 & 0.014 & 0.609 & 0.986 \\
\hline & & GPCM & 0.036 & 0.980 & 0.990 & 0.005 & 0.950 & 0.990 \\
\hline
\end{tabular}


Table 4.10 Empirical Type I Error Rate for RISE, PARSCALE's $G^{2}$ and IRTFIT's $S$ - $X^{2}$ Statistic, Positivey-Skewed Ability Distribution, 3PLM/2PLM/GRM Model Combination

\begin{tabular}{|c|c|c|c|c|c|c|c|c|}
\hline $\begin{array}{c}\text { Sample } \\
\text { Size }\end{array}$ & $\begin{array}{c}\text { Test } \\
\text { Length }\end{array}$ & Model & $\begin{array}{l}\text { RISE } \\
(0.05)\end{array}$ & $\begin{array}{c}G^{2} \\
(0.05)\end{array}$ & $\begin{array}{c}S-X^{2} \\
(0.05)\end{array}$ & $\begin{array}{l}\text { RISE } \\
(0.01)\end{array}$ & $\begin{array}{c}G^{2} \\
(0.01)\end{array}$ & $\begin{array}{c}S-X^{2} \\
(0.01)\end{array}$ \\
\hline \multirow[t]{6}{*}{500} & \multirow[t]{3}{*}{30} & 3PLM & 0.037 & 0.079 & 0.254 & 0.011 & 0.021 & 0.111 \\
\hline & & 2PLM & 0.042 & 0.081 & 0.348 & 0.009 & 0.040 & 0.160 \\
\hline & & GRM & 0.039 & 0.096 & 0.720 & 0.008 & 0.032 & 0.668 \\
\hline & \multirow[t]{3}{*}{60} & 3PLM & 0.035 & 0.078 & 0.053 & 0.012 & 0.025 & 0.029 \\
\hline & & 2PLM & 0.042 & 0.074 & 0.042 & 0.004 & 0.021 & 0.007 \\
\hline & & GRM & 0.031 & 0.108 & 0.198 & 0.009 & 0.029 & 0.099 \\
\hline \multirow[t]{6}{*}{2,000} & \multirow[t]{3}{*}{30} & 3PLM & 0.039 & 0.155 & 0.897 & 0.002 & 0.078 & 0.756 \\
\hline & & 2PLM & 0.045 & 0.177 & 0.906 & 0.012 & 0.084 & 0.880 \\
\hline & & GRM & 0.047 & 0.431 & 0.989 & 0.008 & 0.191 & 0.937 \\
\hline & \multirow[t]{3}{*}{60} & 3PLM & 0.048 & 0.106 & 0.476 & 0.011 & 0.037 & 0.281 \\
\hline & & 2PLM & 0.049 & 0.098 & 0.501 & 0.010 & 0.032 & 0.384 \\
\hline & & GRM & 0.050 & 0.344 & 0.834 & 0.007 & 0.145 & 0.795 \\
\hline \multirow{6}{*}{10,000} & \multirow[t]{3}{*}{30} & 3PLM & 0.030 & 0.798 & 0.997 & 0.008 & 0.679 & 0.875 \\
\hline & & 2PLM & 0.041 & 0.992 & 0.997 & 0.009 & 0.961 & 0.834 \\
\hline & & GRM & 0.054 & 0.998 & 0.991 & 0.015 & 0.906 & 0.821 \\
\hline & \multirow[t]{3}{*}{60} & 3PLM & 0.039 & 0.434 & 0.974 & 0.008 & 0.302 & 0.924 \\
\hline & & 2PLM & 0.049 & 0.876 & 0.899 & 0.010 & 0.547 & 0.826 \\
\hline & & GRM & 0.048 & 1.000 & 0.990 & 0.007 & 1.000 & 0.900 \\
\hline
\end{tabular}


Table 4.11 Empirical Detection Rate for RISE, PARSCALE's $G^{2}$ and IRTFIT's S- $X^{2}$ Statistic, Positivey-Skewed Ability Distribution, 3PLM/2PLM/GPCM Model Combination

\begin{tabular}{|c|c|c|c|c|c|c|c|c|}
\hline $\begin{array}{c}\text { Sample } \\
\text { Size }\end{array}$ & $\begin{array}{c}\text { Test } \\
\text { Length }\end{array}$ & Model & $\begin{array}{l}\text { RISE } \\
(0.05)\end{array}$ & $\begin{array}{c}G^{2} \\
(0.05)\end{array}$ & $\begin{array}{c}S-X^{2} \\
(0.05)\end{array}$ & $\begin{array}{c}\text { RISE } \\
(0.01)\end{array}$ & $\begin{array}{c}G^{2} \\
(0.01)\end{array}$ & $\begin{array}{c}S-X^{2} \\
(0.01)\end{array}$ \\
\hline \multirow[t]{6}{*}{500} & 30 & 3PLM & 0.576 & 0.250 & 0.14 & 0.385 & 0.735 & 0.08 \\
\hline & & 2PLM & 0.620 & 0.25 & 0.44 & 0.260 & 9. & 0.154 \\
\hline & & GPCM & 0.602 & & & 0.345 & & \\
\hline & 60 & 3PLM & 0.488 & & 0.15 & 0.390 & & 0.0 \\
\hline & & 2PLM & 0.593 & $0.3 \theta$ & 0.060 & 0.347 & 0.22 & 0.050 \\
\hline & & GPCM & 0.607 & & & 0.500 & & \\
\hline \multirow[t]{6}{*}{2,000} & 30 & 3PLM & 0.766 & & & 0.508 & 0.253 & \\
\hline & & 2PLM & 0.903 & 280 & 0.980 & 0.730 & 0.040 & 78 \\
\hline & & GPCM & 0.917 & & & 0.824 & & \\
\hline & 60 & 3PLM & 0.769 & & & 0.533 & 0.19 & \\
\hline & & 2PLM & 0.821 & 2.33 & 0.610 & 0.706 & 0.040 & 0.330 \\
\hline & & GPCM & 0.905 & & & 0.741 & & \\
\hline \multirow[t]{6}{*}{10,000} & 30 & 3PLM & 0.978 & & & 0.902 & & \\
\hline & & 2PLM & 0.946 & & 090 & 0.918 & 19650 & 990 \\
\hline & & GPCM & 0.999 & & & 0.899 & & \\
\hline & 60 & 3PLM & 0.998 & & & 0.970 & & \\
\hline & & 2PLM & 0.905 & & 0.990 & 0.862 & 1.78 & 0.990 \\
\hline & & GPCM & 1.000 & 1.000 & 0.990 & 0.956 & 0.985 & 0.990 \\
\hline
\end{tabular}

X-ed out cells represent conditions in which the test statistic exhibited inflated Type I error rates. 
Table 4.12 Empirical Detection Rate for RISE, PARSCALE's $G^{2}$ and IRTFIT's $S$ - $X^{2}$ Statistic, Positivey-Skewed Ability Distribution, 3PLM/2PLM/GRM Model Combination

\begin{tabular}{|c|c|c|c|c|c|c|c|c|}
\hline $\begin{array}{c}\text { Sample } \\
\text { Size }\end{array}$ & $\begin{array}{c}\text { Test } \\
\text { Length }\end{array}$ & Model & $\begin{array}{l}\text { RISE } \\
(0.05)\end{array}$ & $\begin{array}{c}G^{2} \\
(0.05)\end{array}$ & $\begin{array}{c}S-X^{2} \\
(0.05)\end{array}$ & $\begin{array}{l}\text { RISE } \\
(0.01)\end{array}$ & $\begin{array}{c}G^{2} \\
(0.01)\end{array}$ & $\begin{array}{c}S-X^{2} \\
(0.01)\end{array}$ \\
\hline \multirow[t]{6}{*}{500} & \multirow[t]{3}{*}{30} & 3PLM & 0.478 & 0.08 & 0.21 & 0.365 & प.t. & 0.1 \\
\hline & & 2PLM & 0.676 & & 0.34 & 0.404 & 0.078 & . \\
\hline & & GRM & 0.656 & & & 0.487 & & \\
\hline & \multirow[t]{3}{*}{60} & 3PLM & 0.669 & & 0.119 & 0.490 & & 0.10 \\
\hline & & 2PLM & 0.624 & 2.15 & 0.080 & 0.320 & 46 & 0.040 \\
\hline & & GRM & 0.599 & & & 0.371 & & \\
\hline \multirow[t]{6}{*}{2,000} & \multirow[t]{3}{*}{30} & 3PLM & 0.788 & & & 0.574 & & \\
\hline & & 2PLM & 0.901 & & & 0.668 & & \\
\hline & & GRM & 0.925 & & & 0.812 & & \\
\hline & \multirow[t]{3}{*}{60} & 3PLM & 0.812 & & & 0.730 & & \\
\hline & & 2PLM & 0.795 & & 15 & 0.649 & 0.230 & 1 \\
\hline & & GRM & 0.901 & & & 0.751 & & \\
\hline \multirow[t]{6}{*}{10,000} & \multirow[t]{3}{*}{30} & 3PLM & 0.997 & & & 0.889 & & \\
\hline & & 2PLM & 0.984 & & 0.87 & 0.810 & & 78 \\
\hline & & GRM & 0.995 & & & 0.990 & & \\
\hline & \multirow[t]{3}{*}{60} & 3PLM & 0.998 & & & 0.940 & & \\
\hline & & 2PLM & 0.900 & e. & 0.86 & 0.831 & & \\
\hline & & GRM & 0.999 & 1.000 & 0.875 & 0.939 & 0.990 & 0.802 \\
\hline
\end{tabular}

X-ed out cells represent conditions in which the test statistic exhibited inflated Type I error rates. 
Table 4.13 The Difference of The Difference of Score Distributions Before and After Replacement in Simulation Study

\begin{tabular}{cccc}
\hline Cuts & RISE & $G^{2}$ & $S-X^{2}$ \\
& $(0.005)$ & $(0.005)$ & $(0.005)$ \\
\hline 40 & 0.002 & 0.001 & 0.001 \\
60 & 0.001 & 0.000 & 0.001 \\
80 & 0.001 & 0.001 & 0.000 \\
\hline
\end{tabular}


Table 4.14 The Difference of The Difference of TCCs Before and After Replacement in Simulation Study

\begin{tabular}{cc}
\hline Cuts & RISE \\
& $(0.5)$ \\
\hline-1.41 & 0.421 \\
-0.33 & 0.497 \\
1.11 & 0.499 \\
\hline
\end{tabular}


Table 4.15 Misfitting Items Detected by Each Fit Statistic at Each Sample Size in Empirical Study

\begin{tabular}{|c|c|c|c|c|}
\hline $\begin{array}{c}\text { Sample } \\
\text { Size }\end{array}$ & $\begin{array}{c}\text { Fit } \\
\text { Statistics }\end{array}$ & Number & Percentage & Item ID \\
\hline \multirow[t]{3}{*}{500} & RISE & 1 & $2 \%$ & 18 \\
\hline & $G^{2}$ & 5 & $12 \%$ & $12,16,17,23,30$ \\
\hline & $S-X^{2}$ & 0 & $0 \%$ & No Items Flagged \\
\hline \multirow[t]{3}{*}{2,000} & RISE & 3 & $7 \%$ & $12,18,37$ \\
\hline & $G^{2}$ & 8 & $19 \%$ & $2,5,10,14,16,37,38,40$ \\
\hline & $S-X^{2}$ & 1 & $2 \%$ & 37 \\
\hline \multirow[t]{3}{*}{10,000} & RISE & 7 & $17 \%$ & $12,16,18,23,30,37,40$ \\
\hline & $G^{2}$ & 40 & $95 \%$ & All Items Except 1 and 15 \\
\hline & $S-X^{2}$ & 8 & $19 \%$ & $12,16,18,23,28,30,37,40$ \\
\hline
\end{tabular}


Table 4.16 The Difference of The Difference of Score Distributions Before and After Replacement in Empirical Study

\begin{tabular}{ccccc}
\hline Sample Size & Cuts & $\begin{array}{c}\text { RISE } \\
(0.005)\end{array}$ & $\begin{array}{c}G^{2} \\
(0.005)\end{array}$ & $\begin{array}{c}S-X^{2} \\
(0.005)\end{array}$ \\
\hline 500 & 25 & 0.003 & 0.002 & \\
& 40 & 0.002 & 0.002 & \\
& 55 & 0.003 & 0.004 & 0.001 \\
2,000 & 25 & 0.003 & 0.001 & 0.001 \\
& 40 & 0.003 & 0.001 & 0.000 \\
& 55 & 0.002 & 0.000 & 0.003 \\
10,000 & 25 & 0.003 & 0.004 & 0.003 \\
& 40 & 0.003 & 0.003 & \\
& 55 & 0.004 & 0.005 & \\
\end{tabular}

X-ed out cells represent conditions in which no misfitting items were detected. 
Figure 4.1. Score Distribution Before and After Replacement of Misfitting Items Detected by RISE.

\section{Score Distribution}

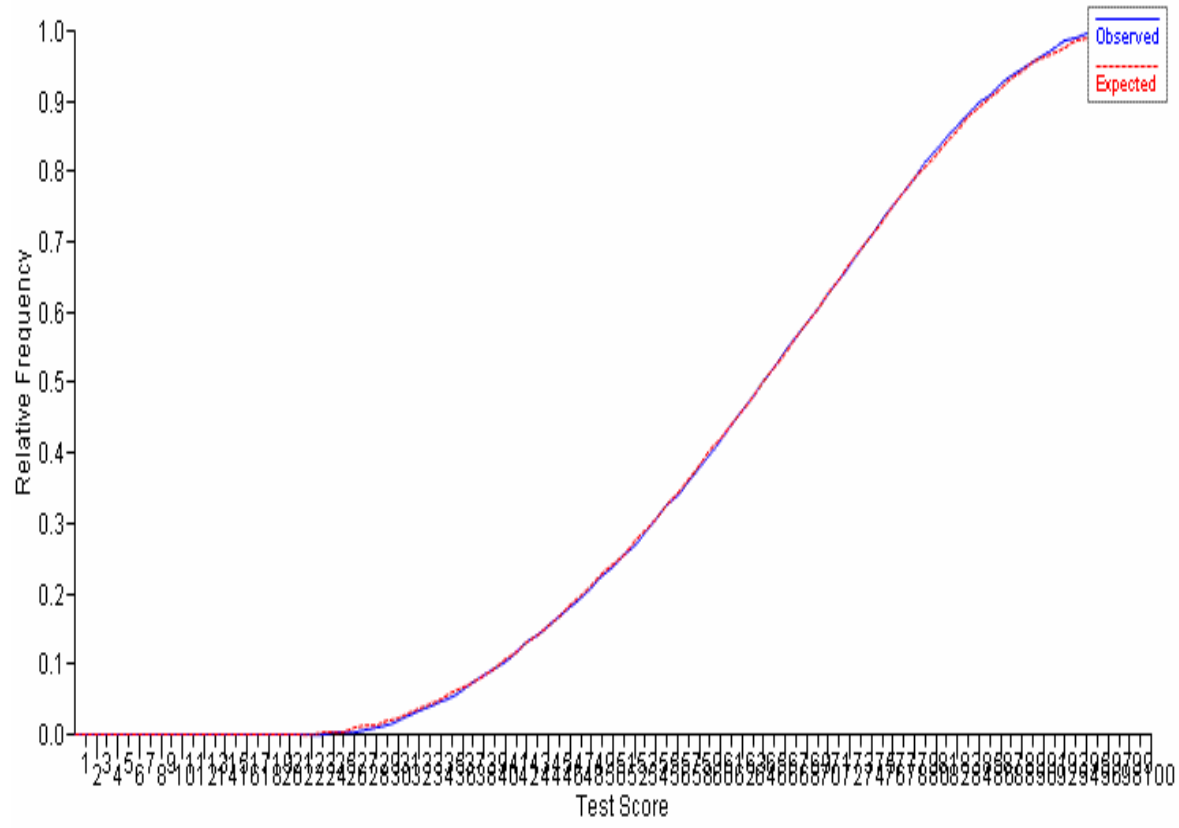

Score Distribution

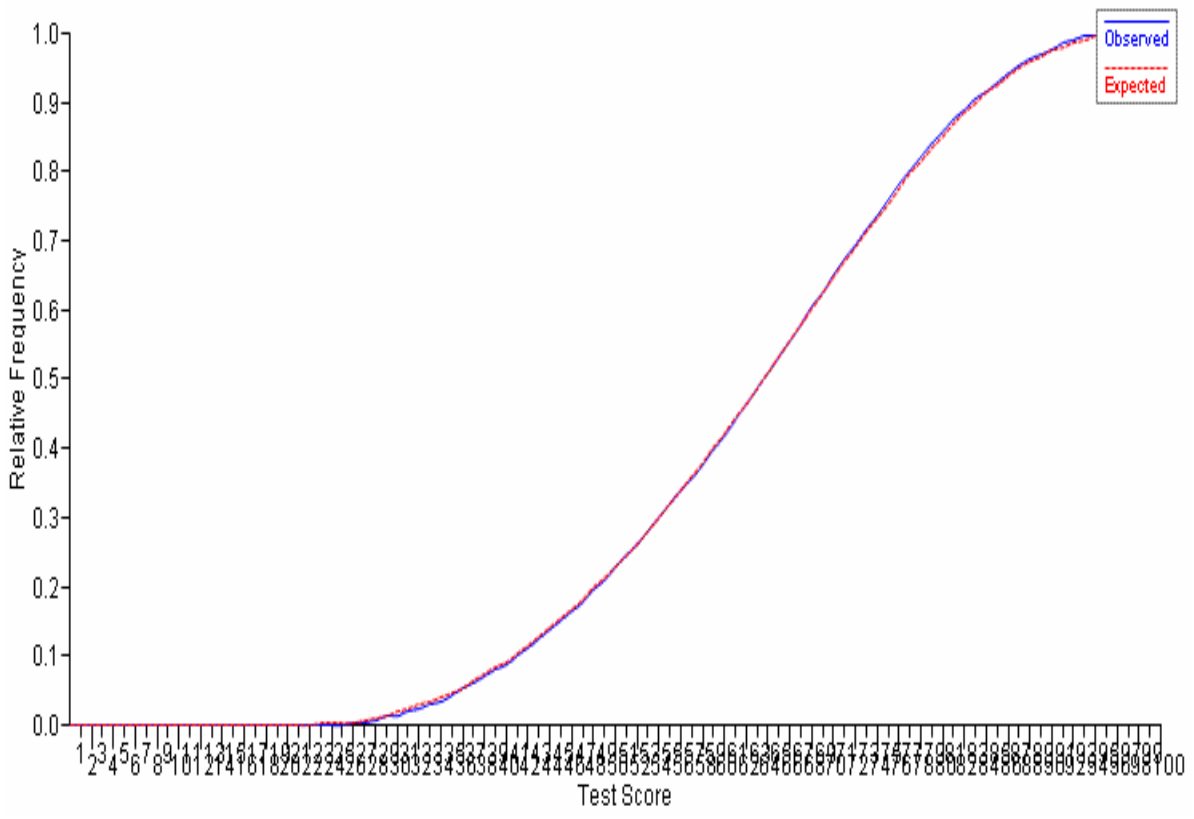


Figure 4.2. Score Distribution Before and After Replacement of Misfitting Items Detected by $G^{2}$.

Score Distribution

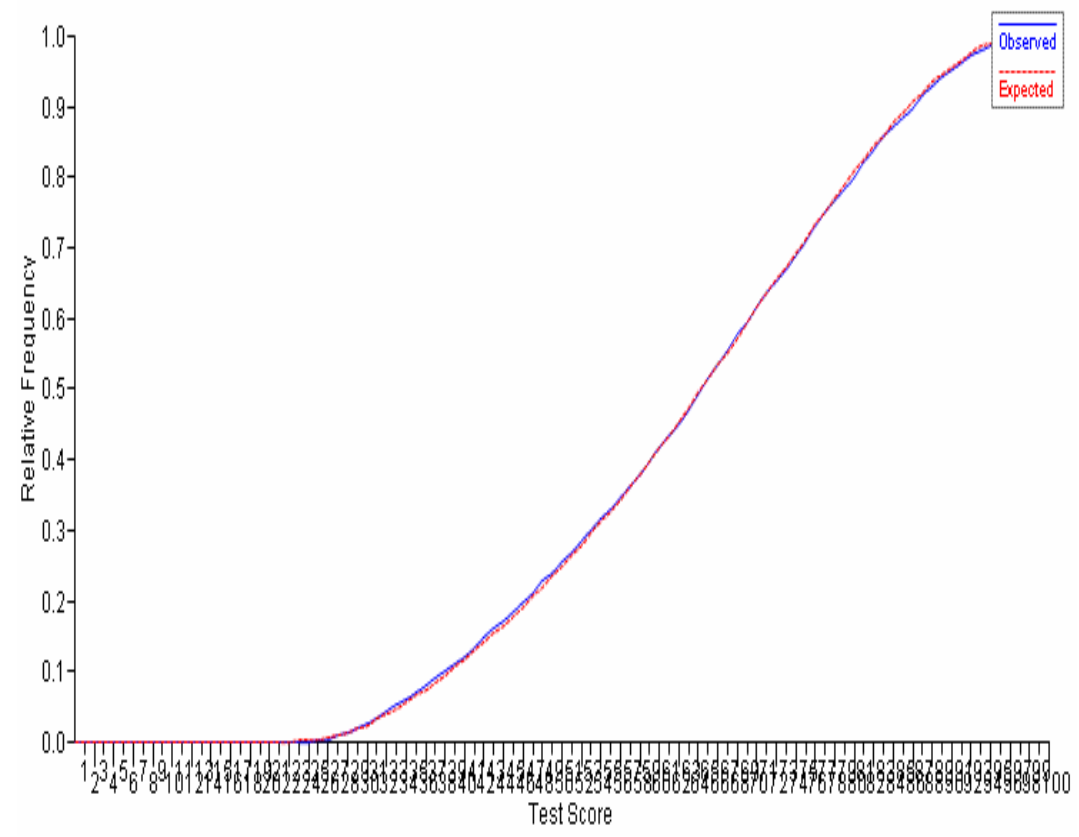

Score Distribution

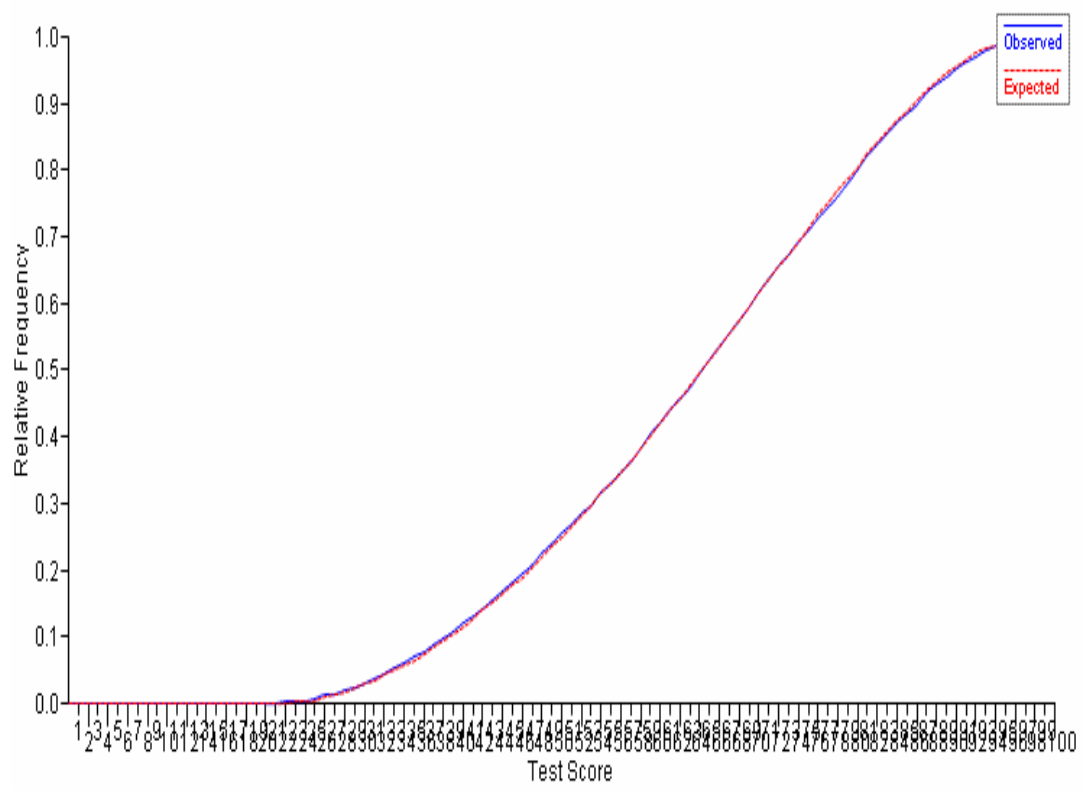


Figure 4.3. Score Distribution Before and After Replacement of Misfitting Items Detected by $S-X^{2}$.

Score Distribution

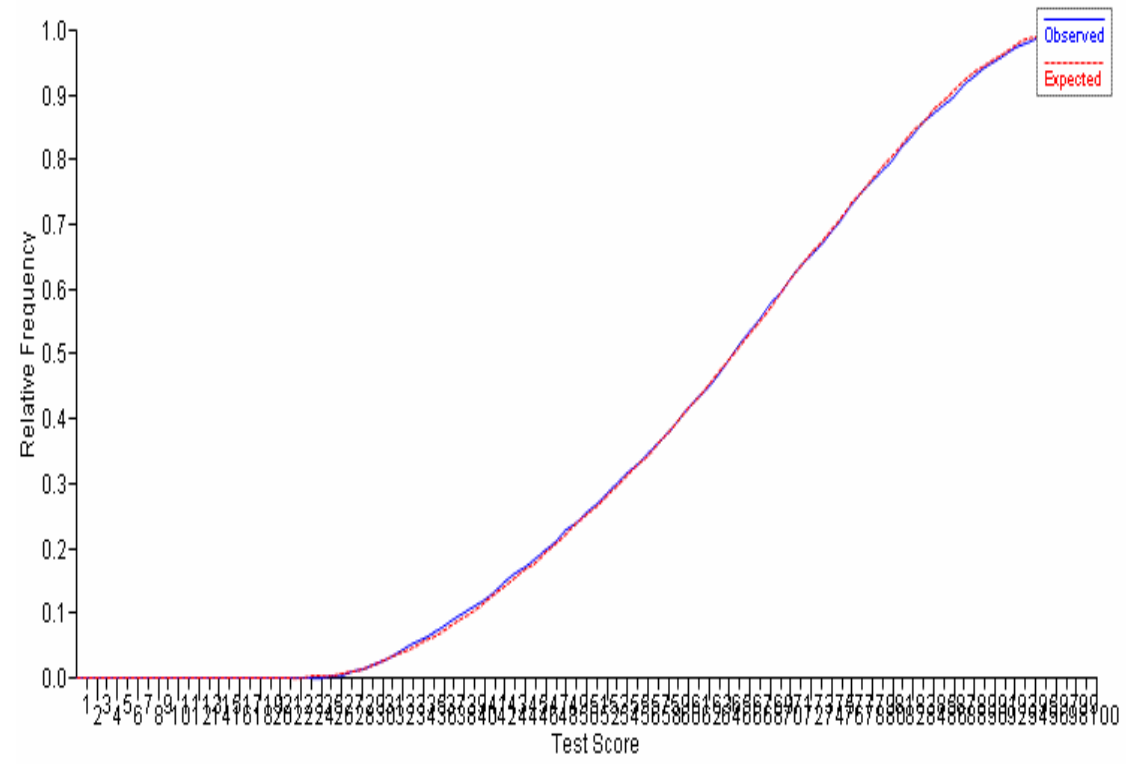

Score Distribution

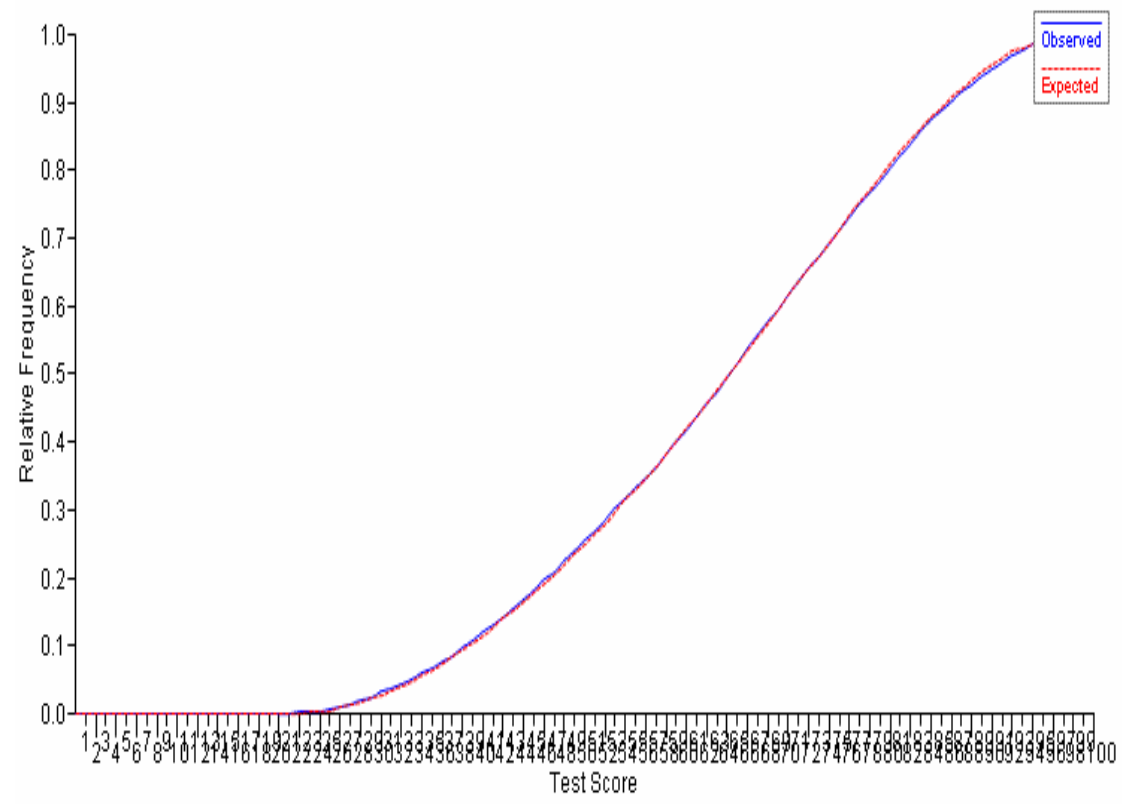


Figure 4.4. TCCs Before and After Replacement of Misfitting Items Detected by RISE.
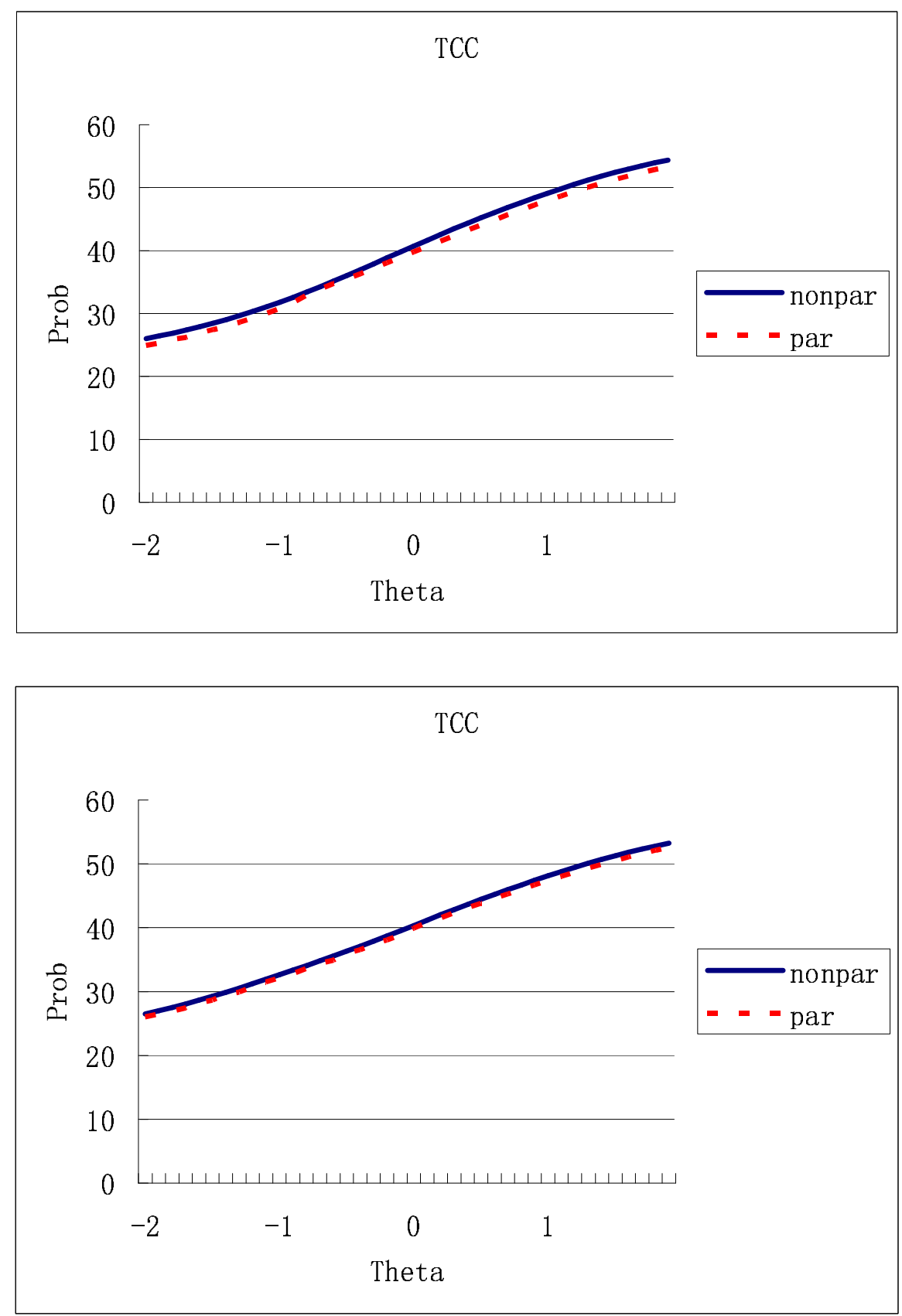
Figure 4.5. Misfitting Items Detected by RISE at Sample Size 500.

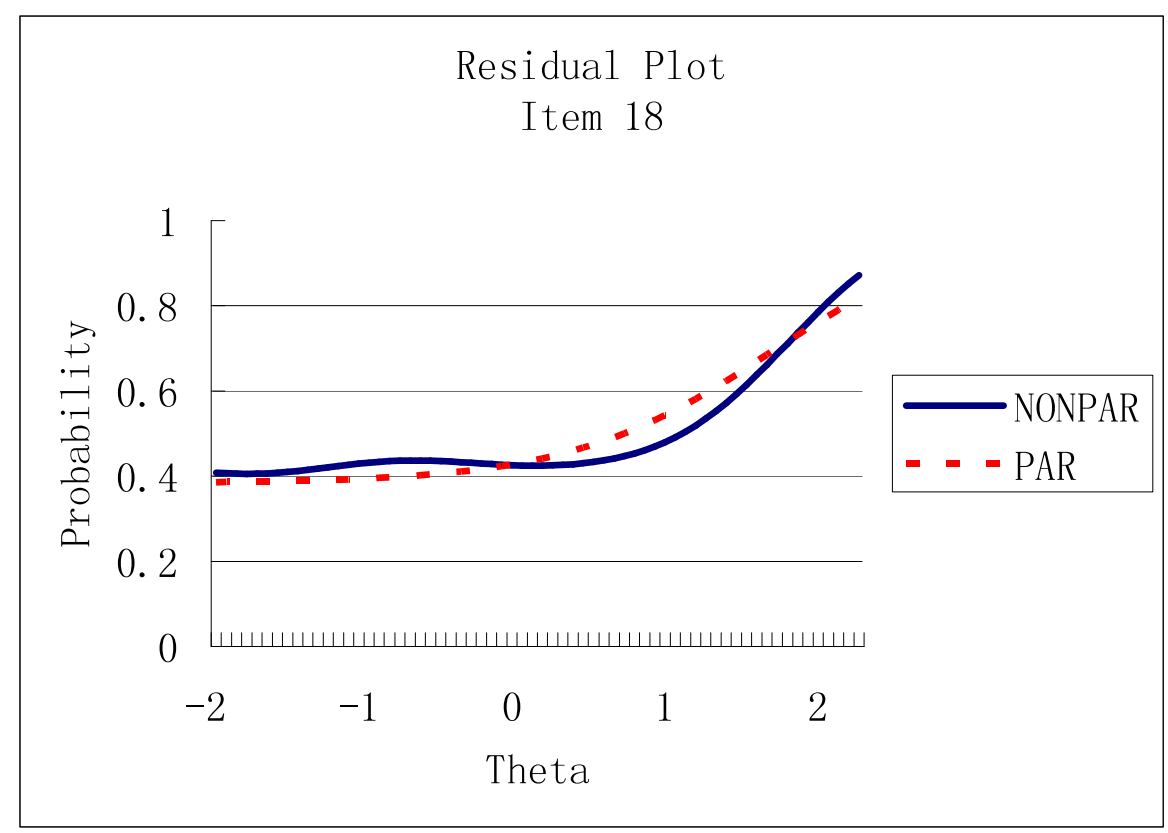


Figure 4.6. Misfitting Items Detected by $G^{2}$ at Sample Size 500.
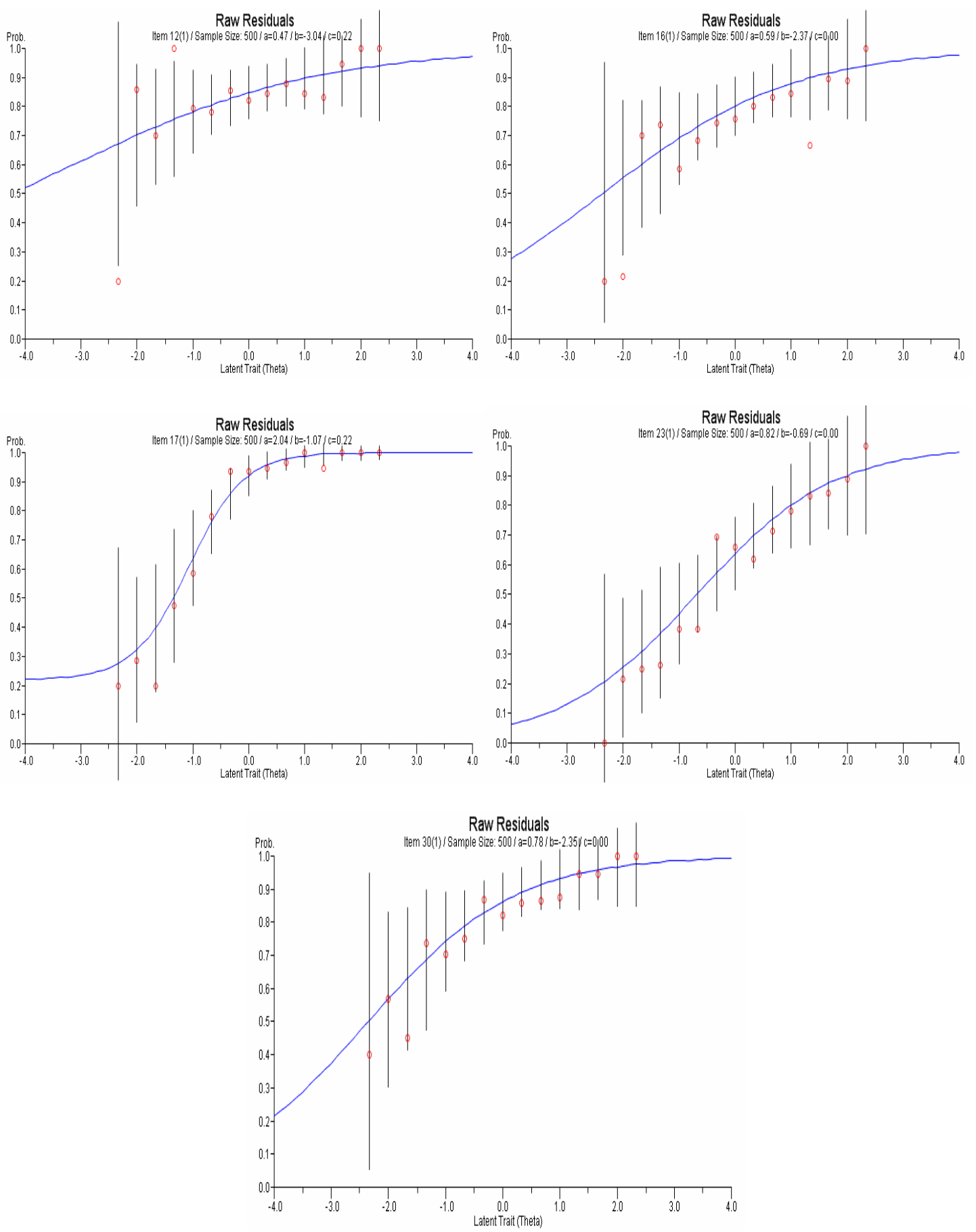
Figure 4.7. Misfitting Items Detected by RISE at Sample Size 2,000.
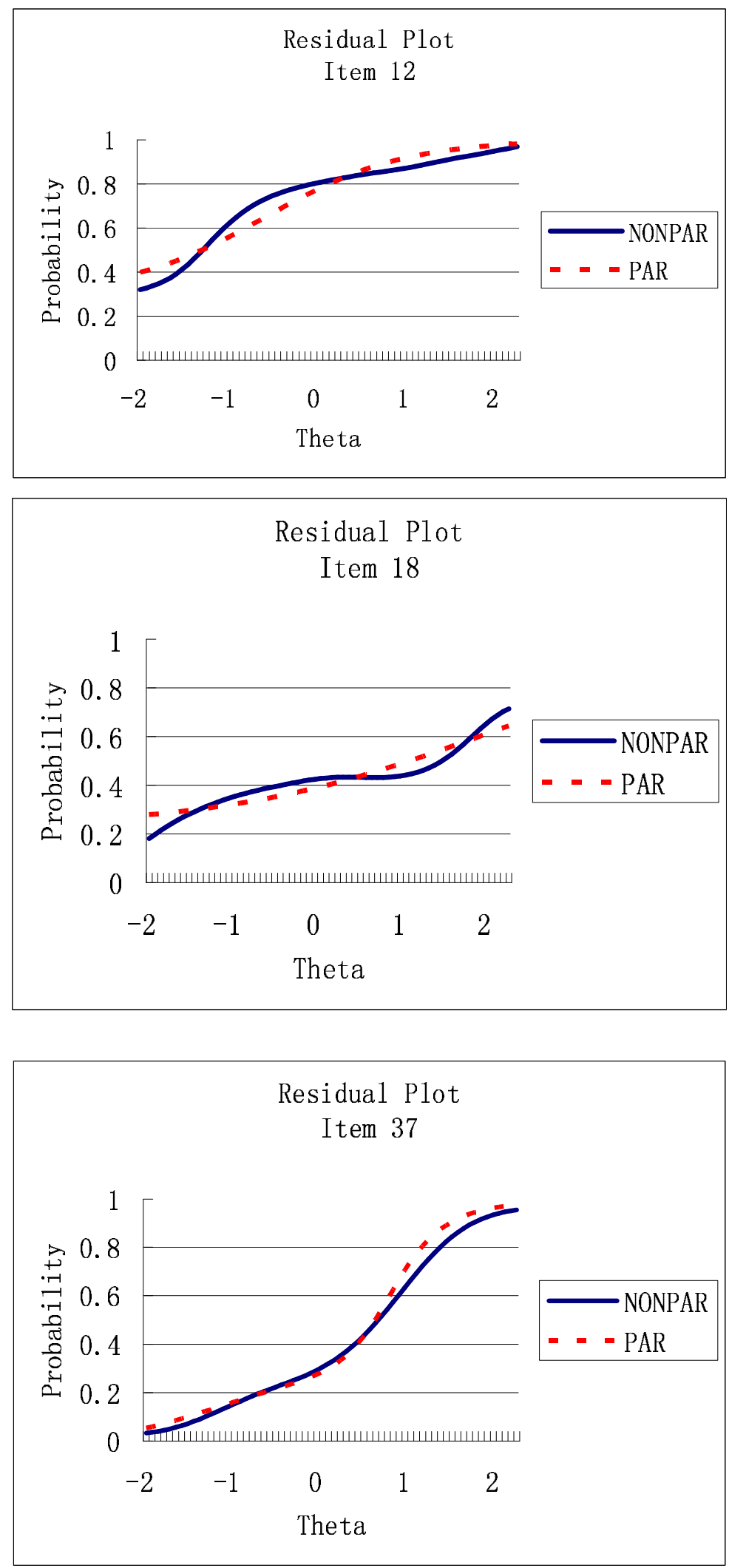
Figure 4.8. Misfitting Items Detected by $G^{2}$ at Sample Size 2,000.
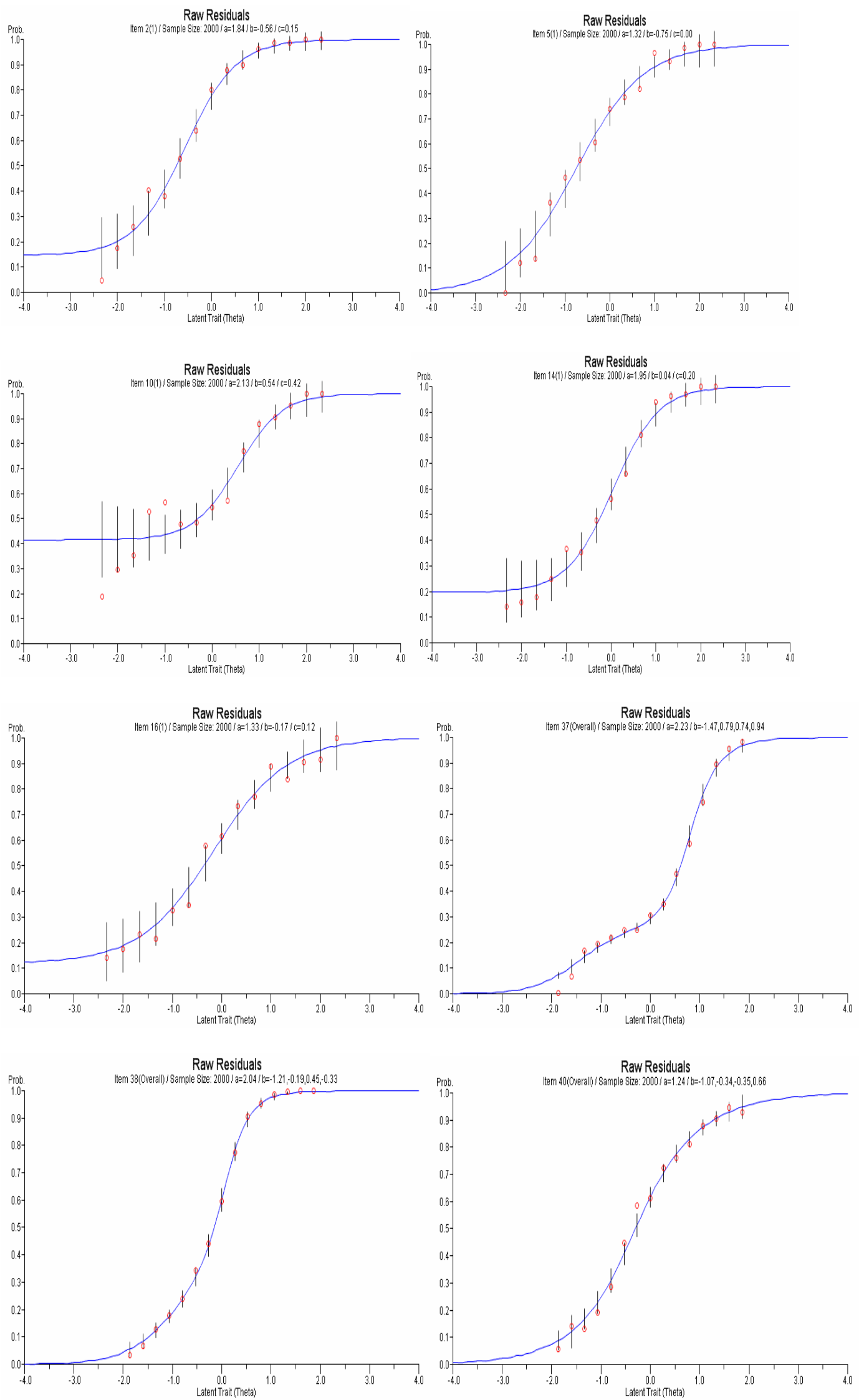
Figure 4.9. Misfitting Items Detected by $S-X^{2}$ at Sample Size 2,000.

\section{Raw Residuals}

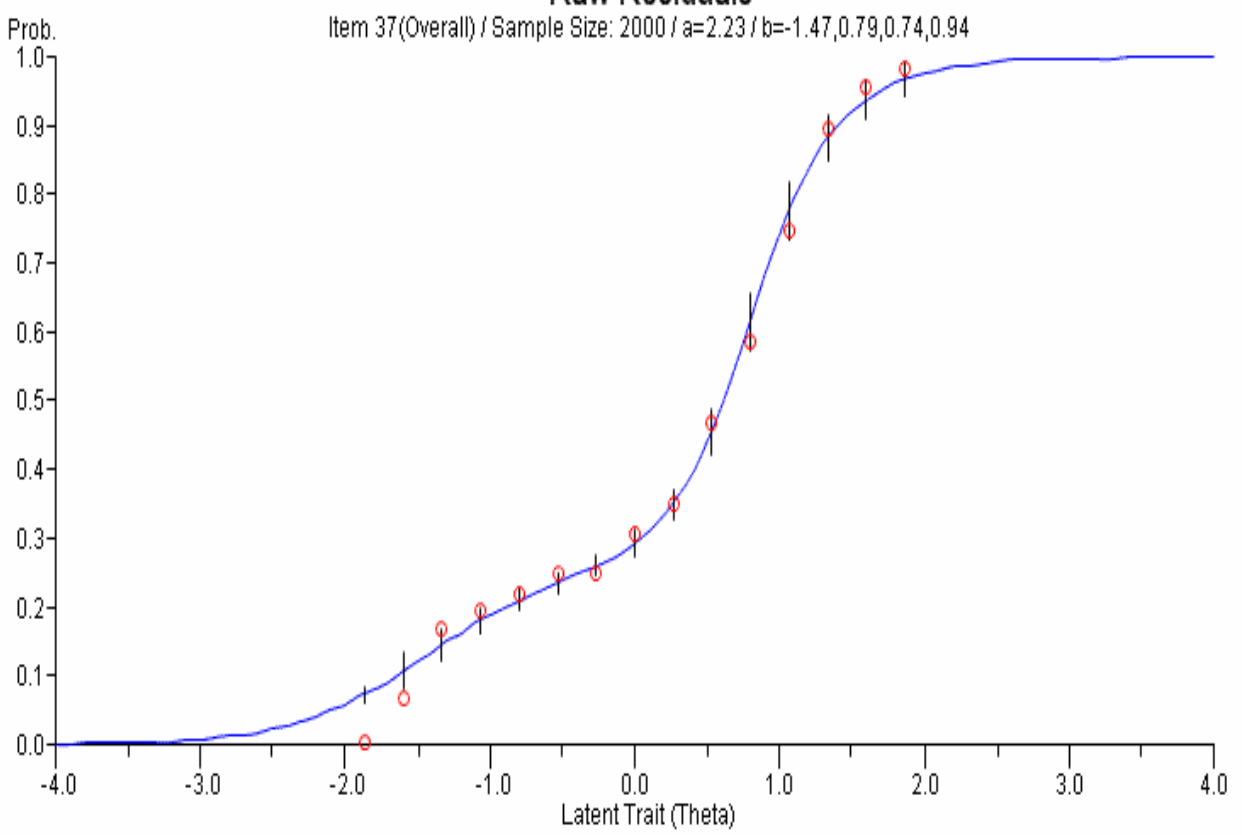


Figure 4.10.Misfitting Items Detected by RISE at Sample Size 10,000.
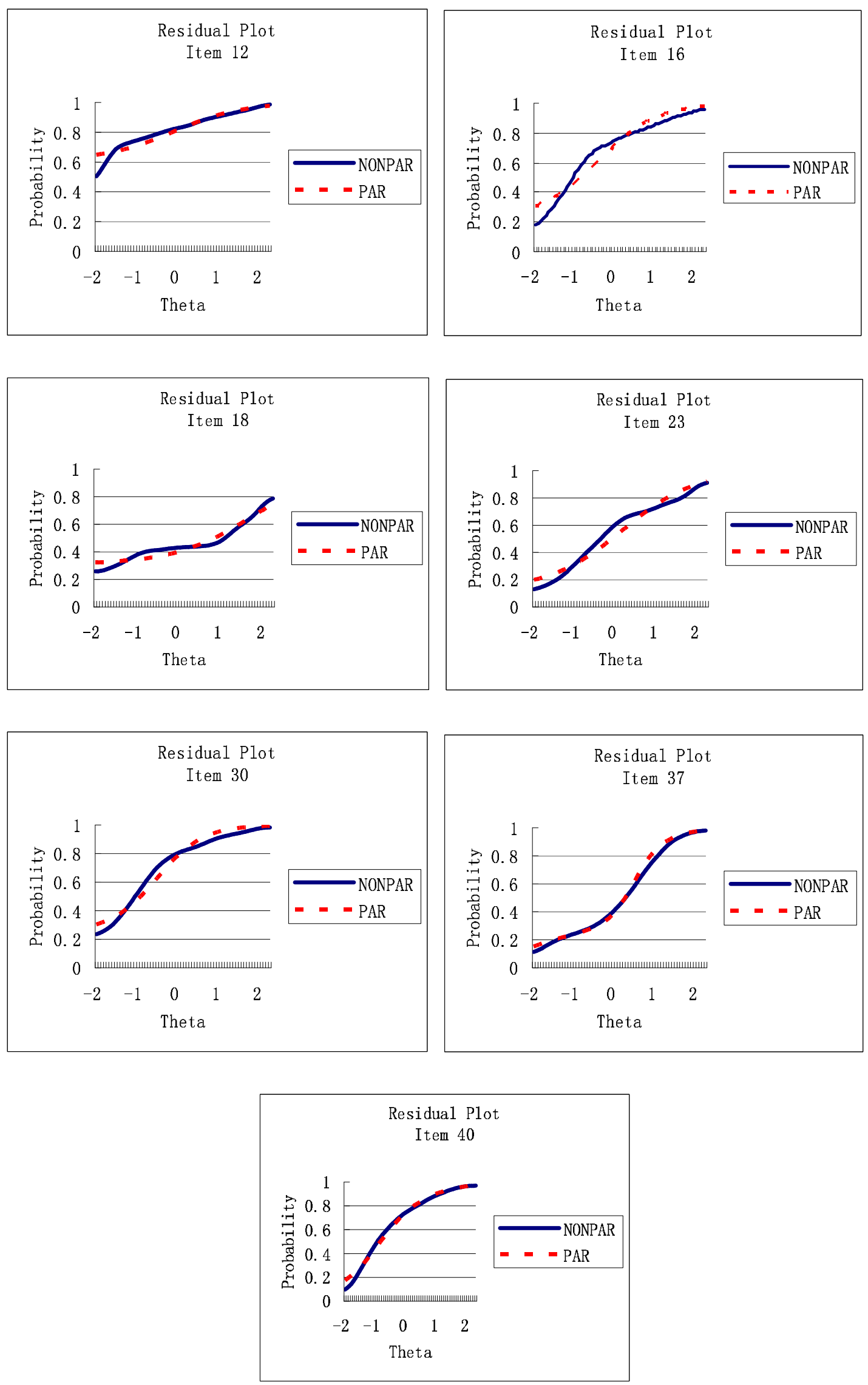
Figure 4.11. Misfitting Items Detected by $G^{2}$ at Sample Size 10,000.
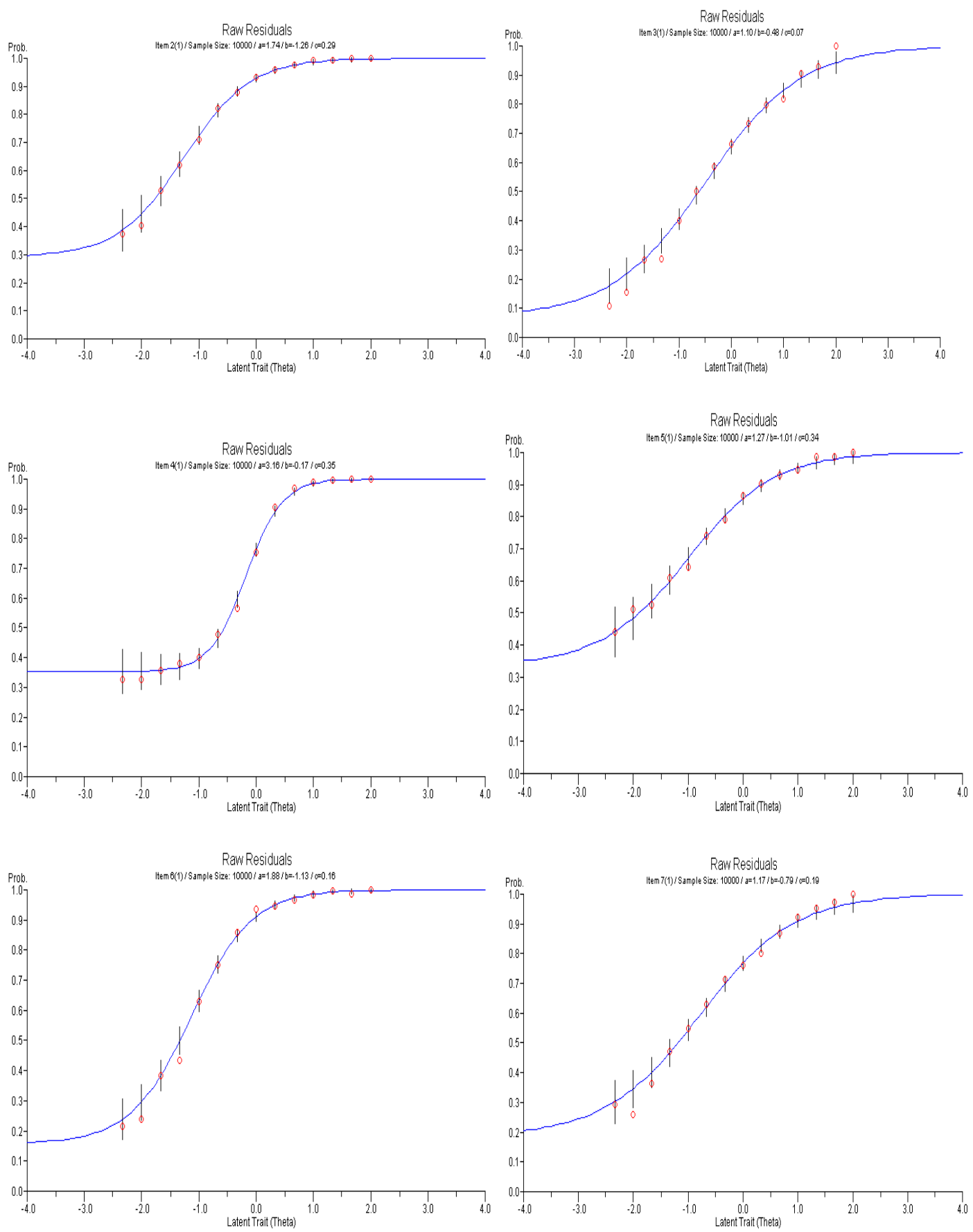

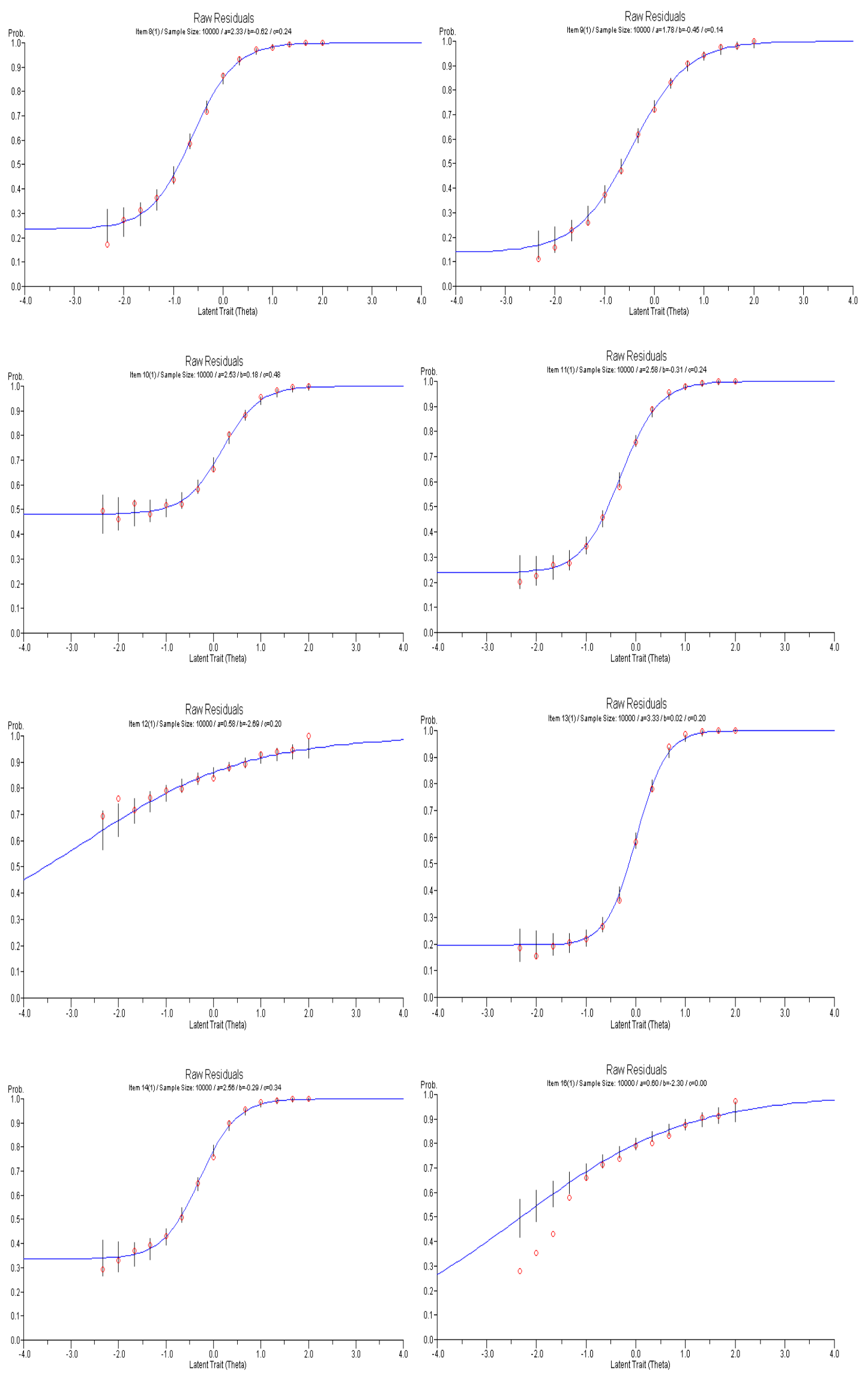

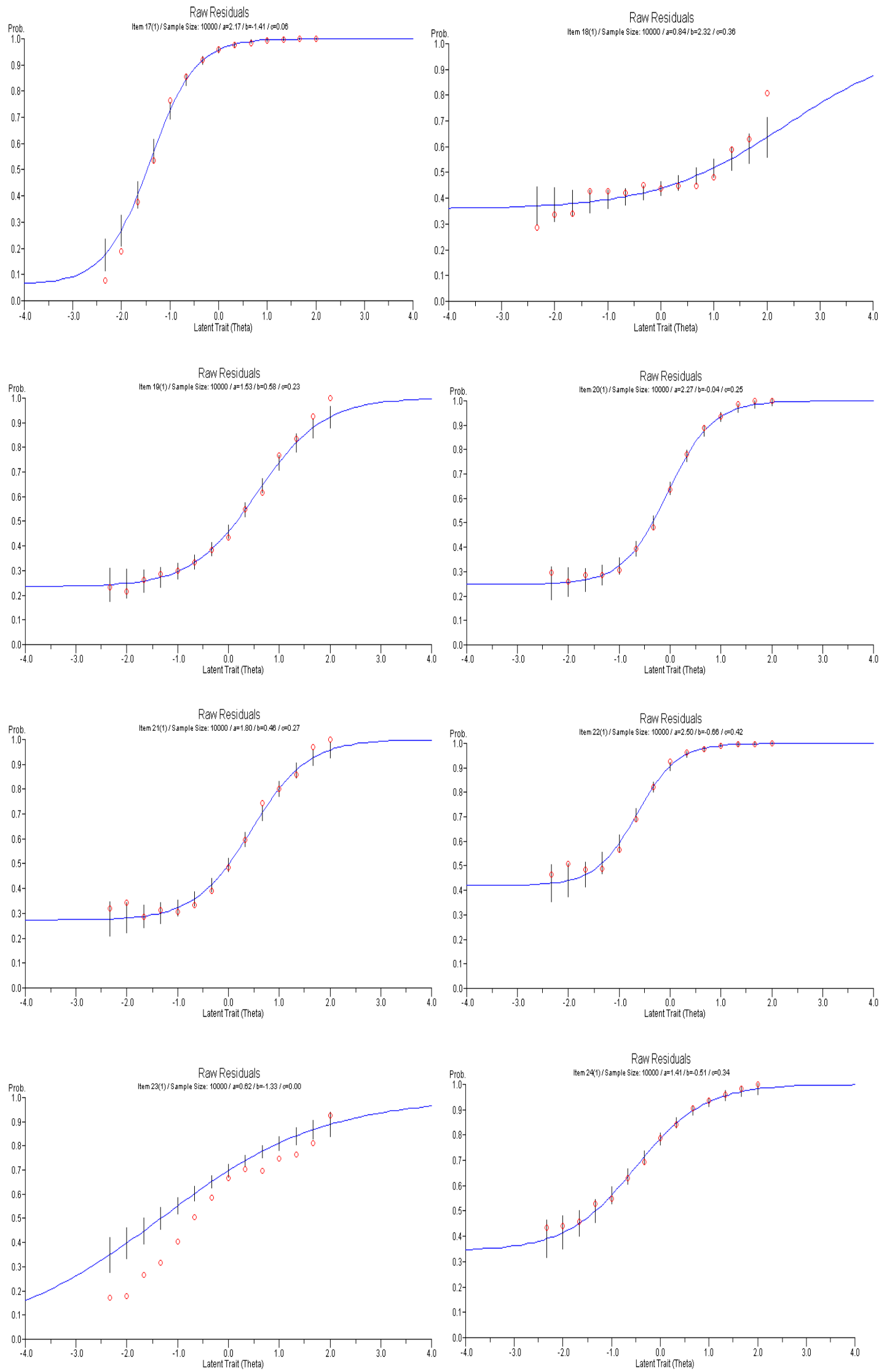

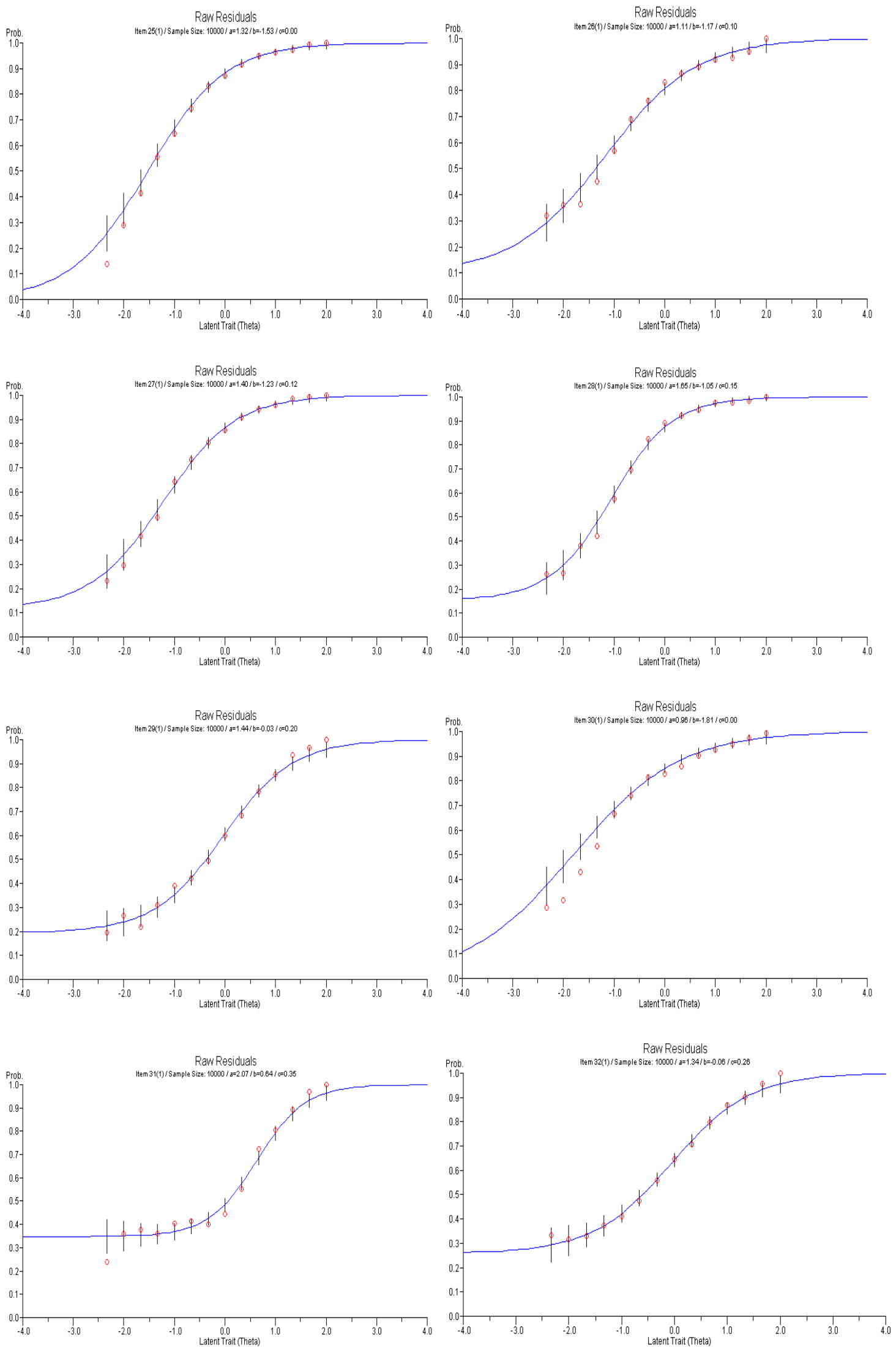
Ram Residuals
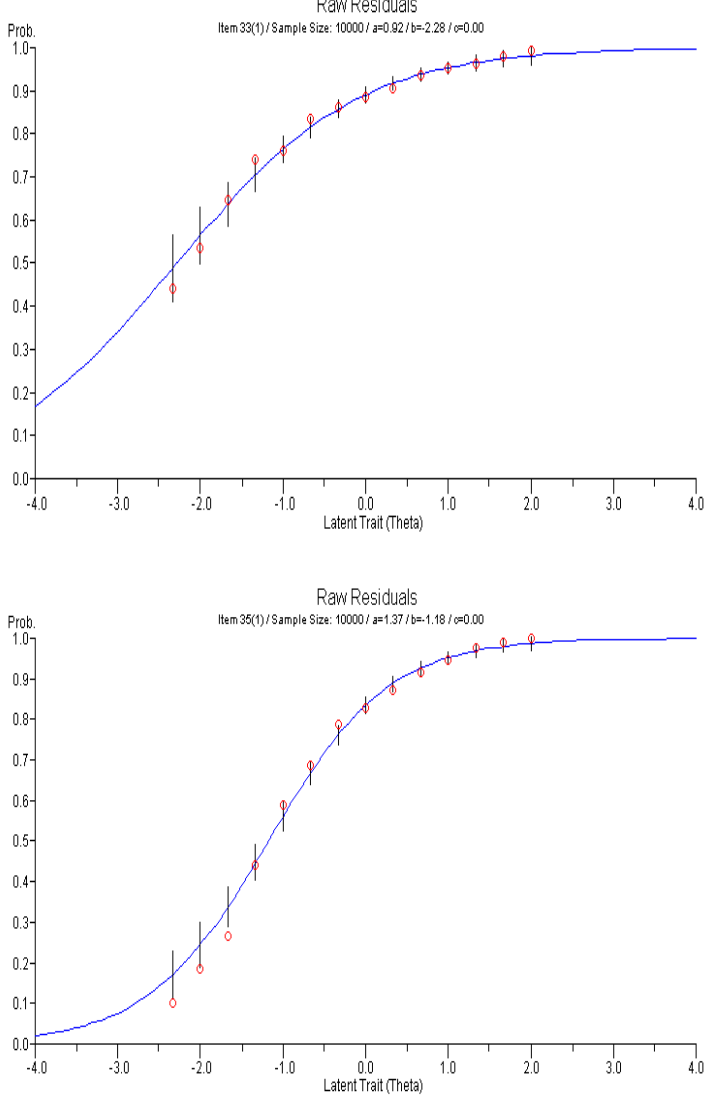

Raw Residuals

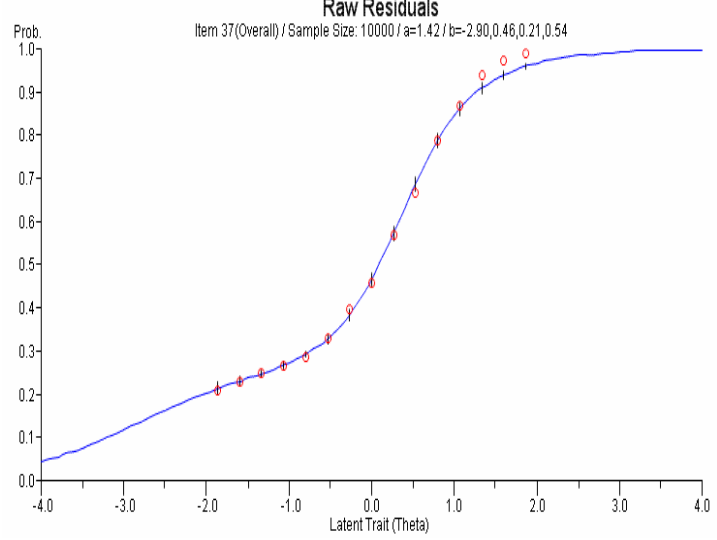

Raw Residuals



Ram Residuals

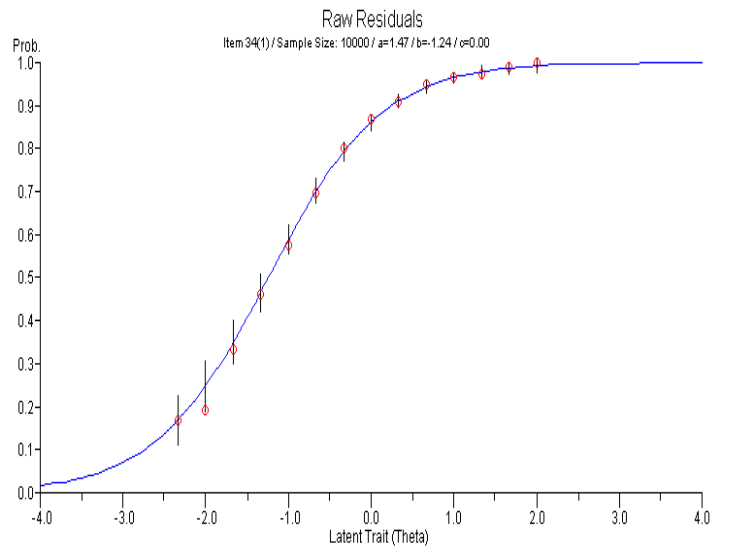

Ram Residuals

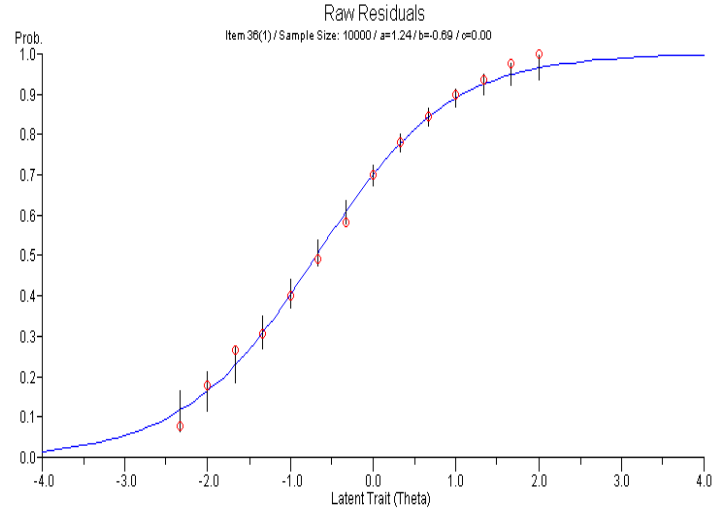

Raww Residuă|s
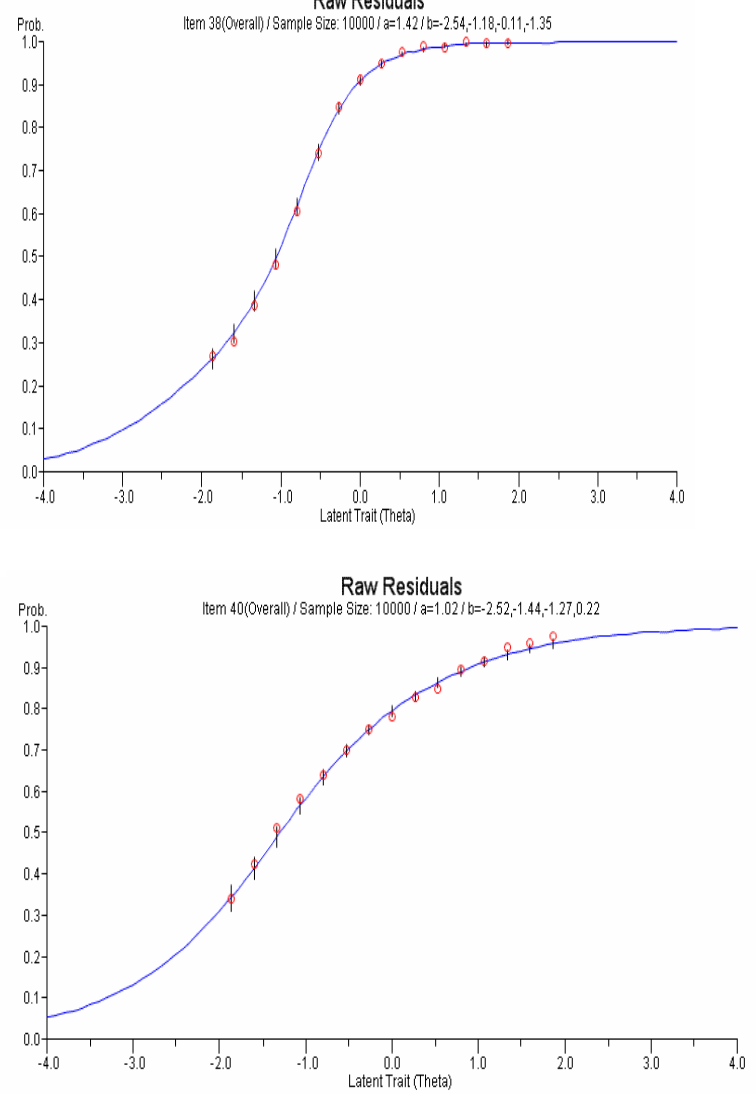

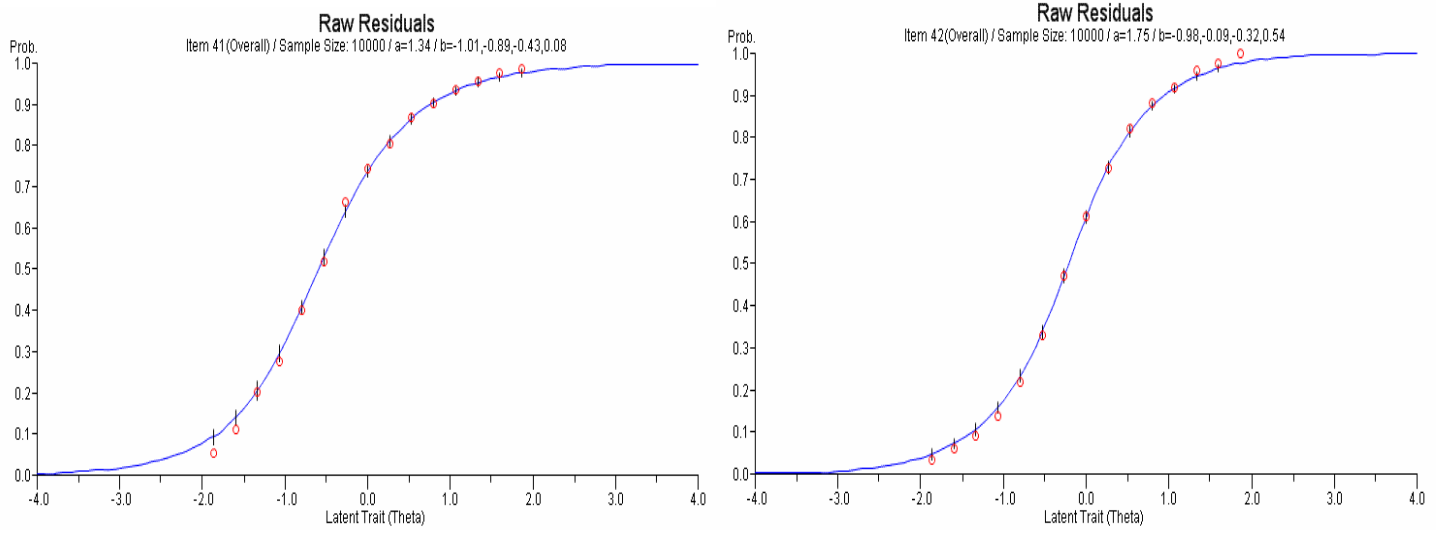
Figure 4.12. Misfitting Items Detected by $S-X^{2}$ at Sample Size 10,000.
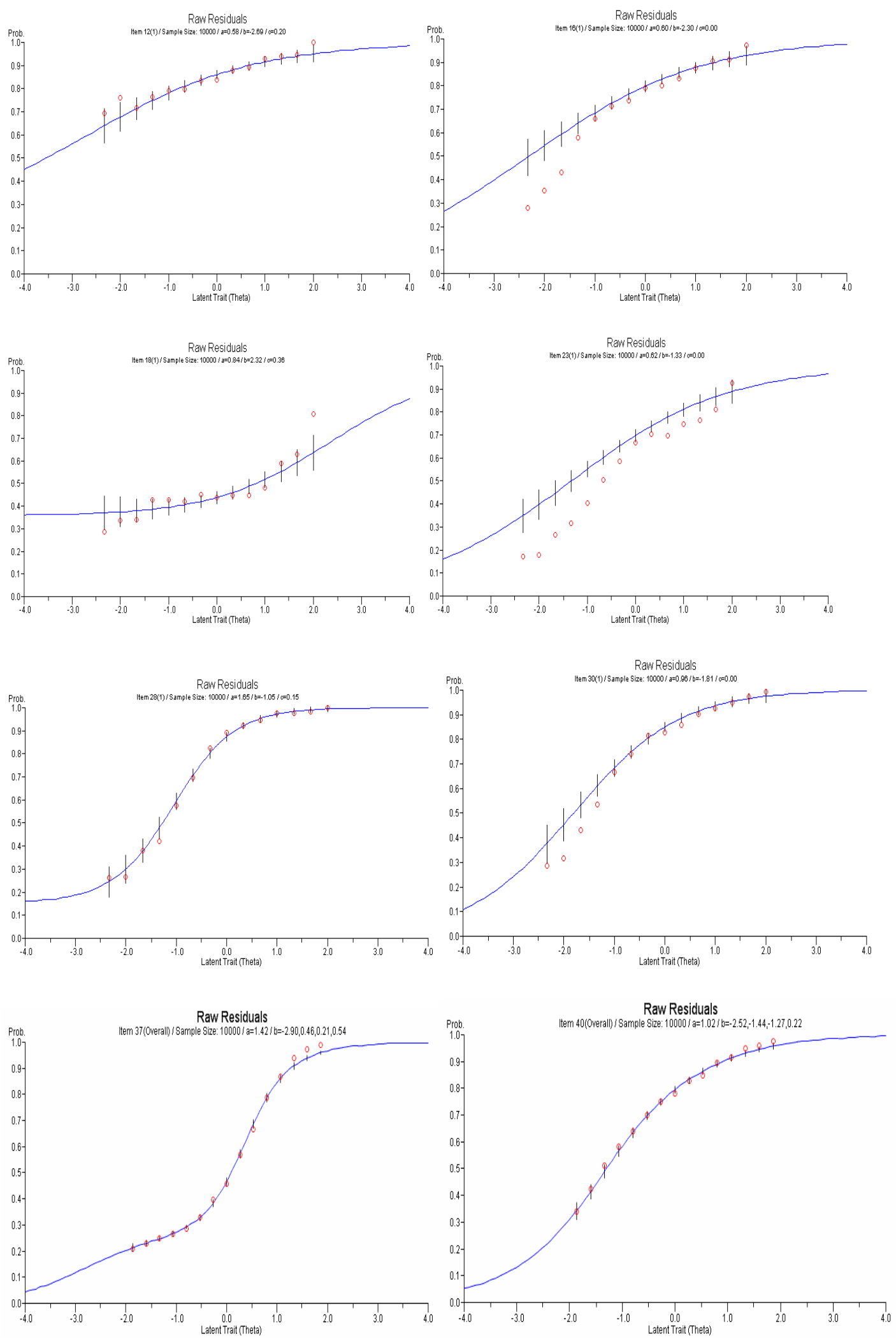
Figure 4.13. Score Distribution Before and After Replacement of Misfitting Items Detected by Three Fit Statistics at Sample Size 500.

\section{RISE}
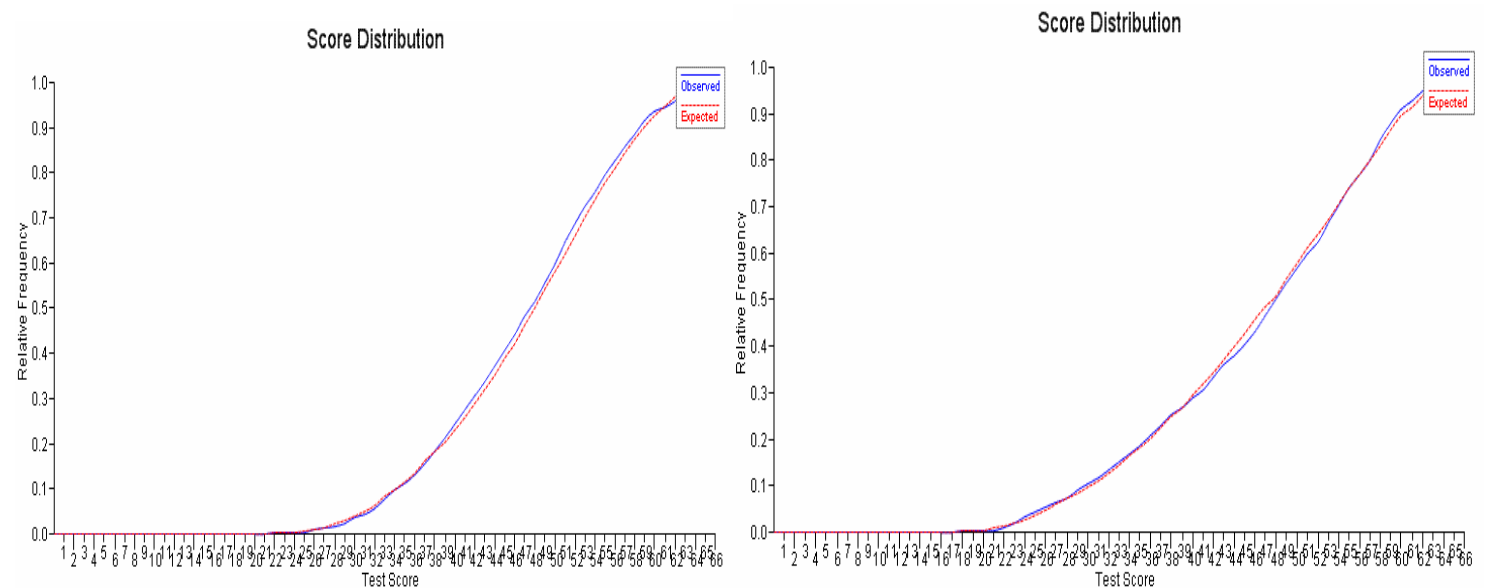

$G^{2}$

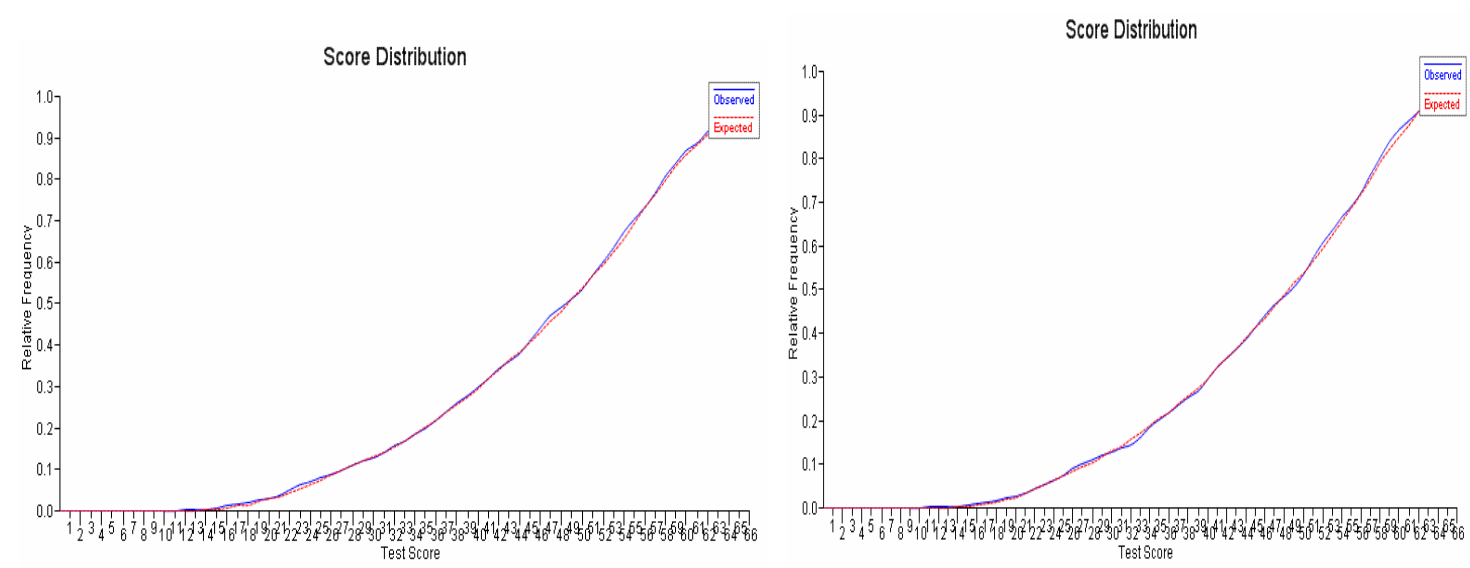

$S-X^{2}$ (no replacement)
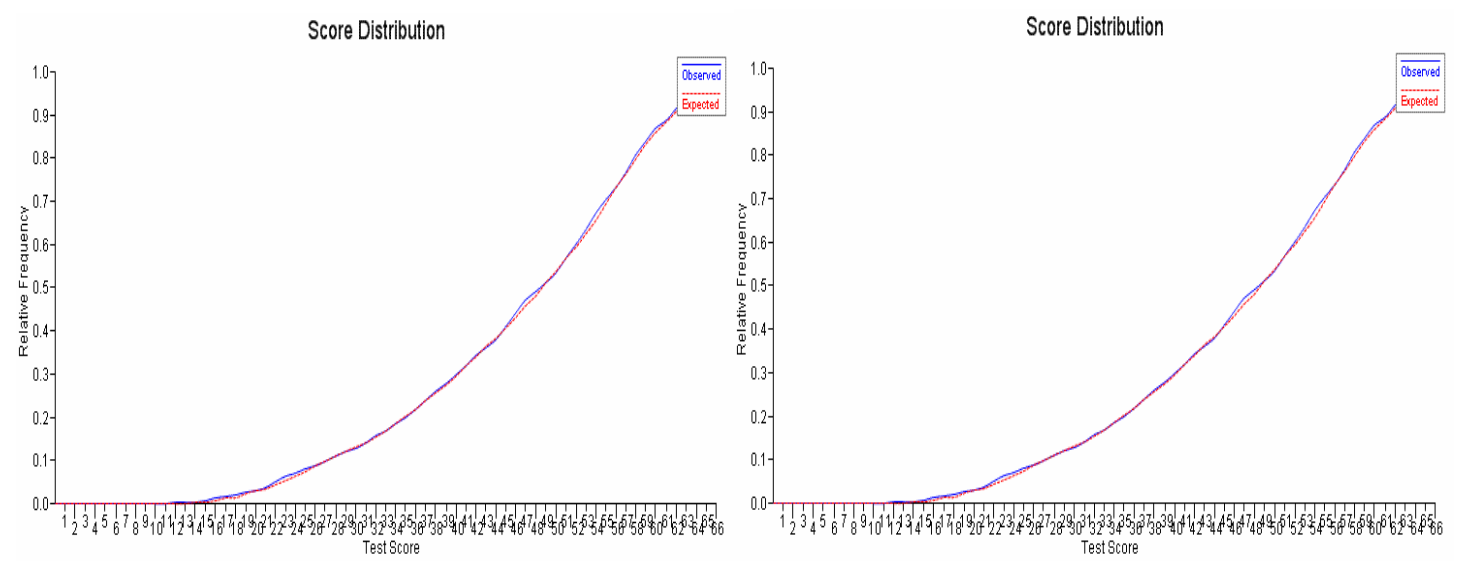
Figure 4.14. Score Distribution Before and After Replacement of Misfitting Items Detected by Three Fit Statistics at Sample Size 2,000.

RISE
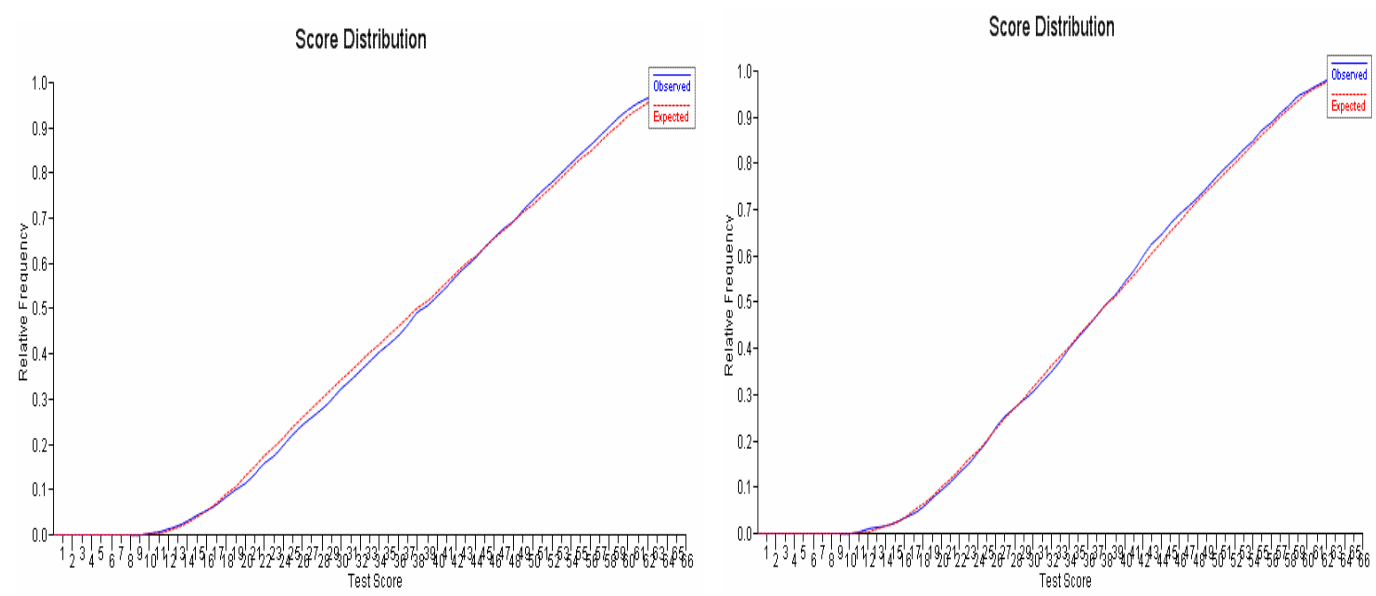

$$
G^{2}
$$

Score Distribution
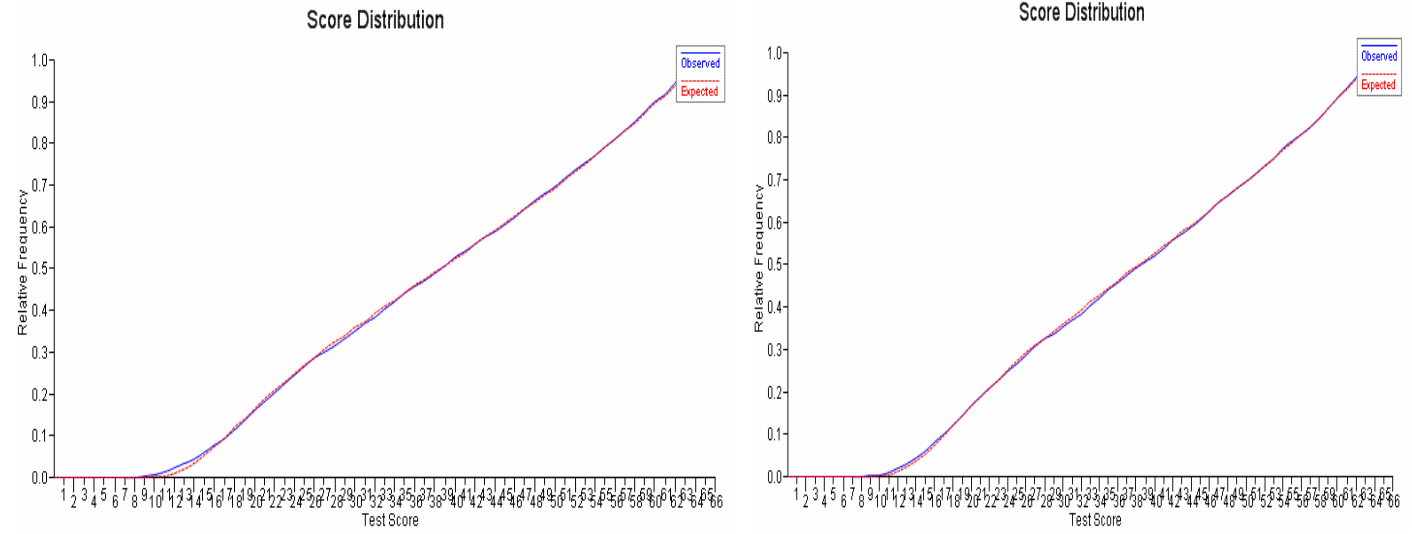

$$
S-X^{2}
$$

Score Distribution

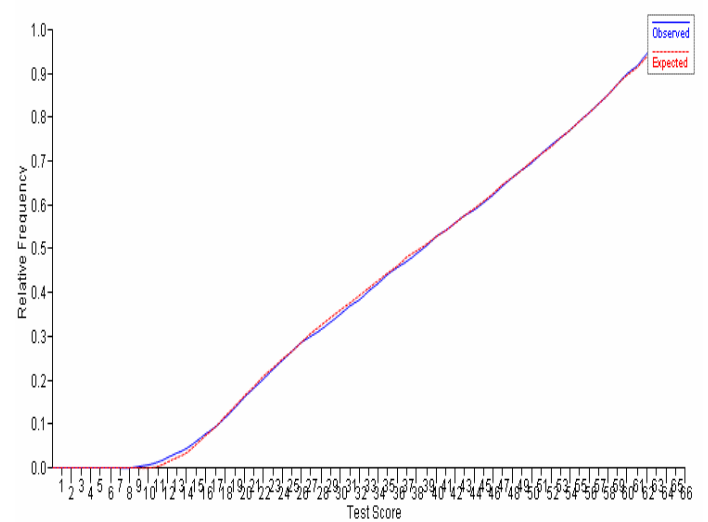

Score Distribution

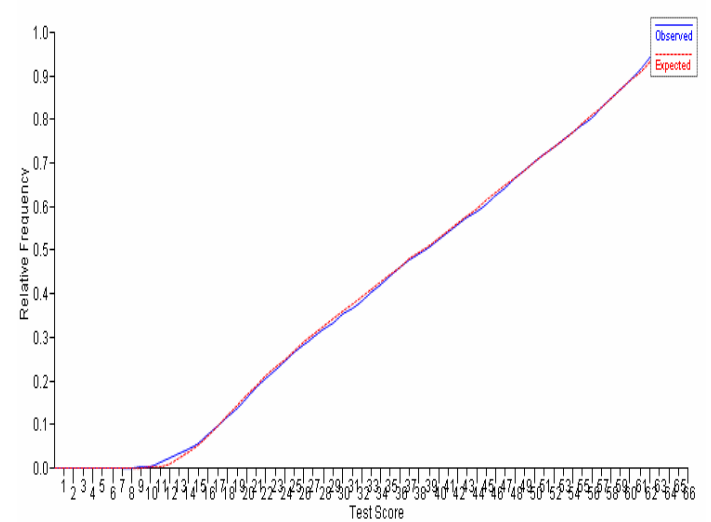


Figure 4.15. Score Distribution Before and After Replacement of Misfitting Items Detected by Three Fit Statistics at Sample Size 10,000.

RISE
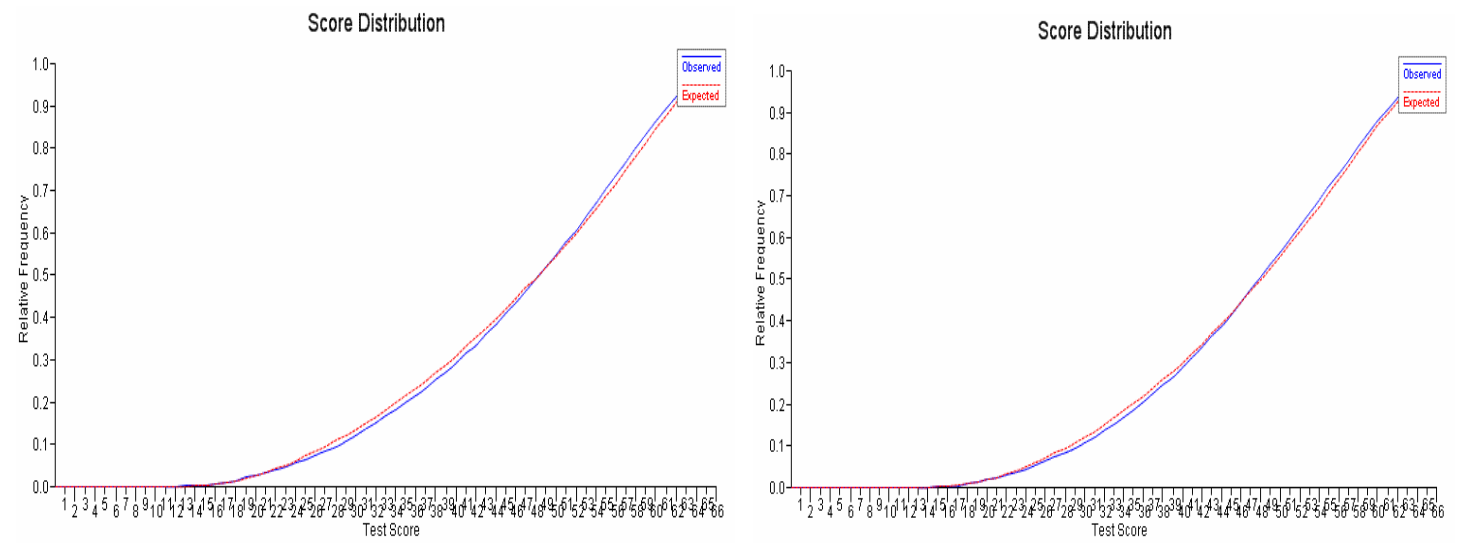

$G^{2}$

Score Distribution

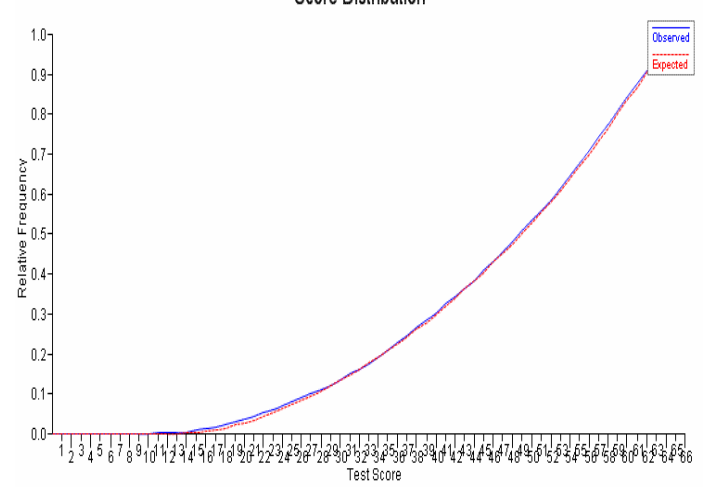

Score Distribution

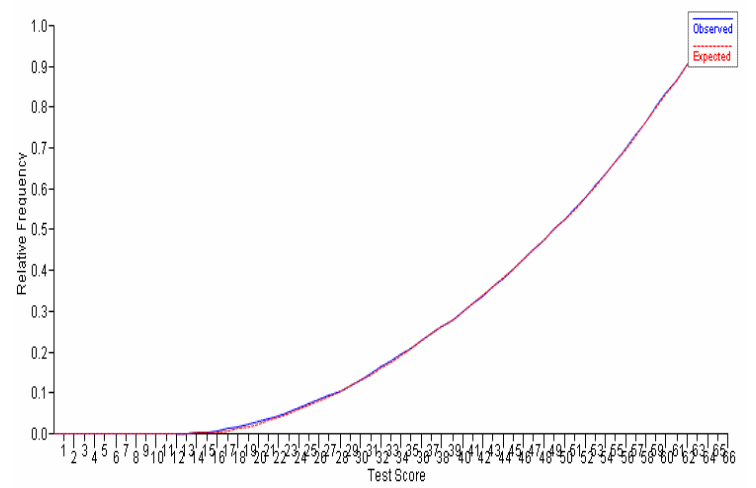

$S-X^{2}$
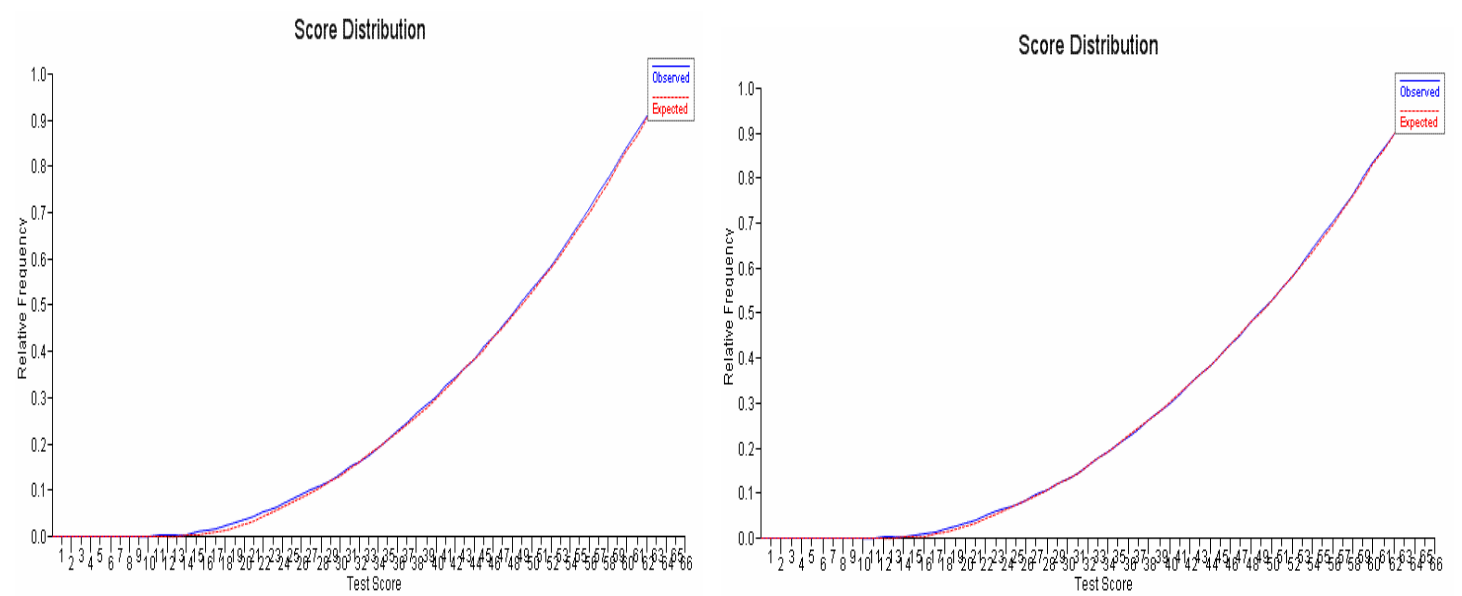


\section{CHAPTER 5}

\section{CONCLUSION}

Model fit is an important aspect in IRT in that misfit between an IRT model and data can undermine the invariance property which is key to all IRT applications such as IRT equating, differential item functioning and computerized adaptive testing. Given the importance of model fit in IRT, the lack of research investigating the influence of misfit as well as the methods to detect misfit, is surprising. Several of the currently available fit statistics (e.g., $G^{2}$ and Yen's $Q_{1}$ ) are unappealing due to inflated Type I error rates and their insensitivity to the location and magnitude of the misfit. In addition, there has been little research on evaluation of model misfit consequences although this topic should be considered seriously in deciding the use of a model in particular situations.

The purpose of this dissertation was to address the above-mentioned two issues in model data fit evaluation procedures. In the aspect of investigating goodness-of-fit statistics, the nonparametric approach was extended and compared to two other commonly used and well-accepted fit statistics through statistical properties and graphical displays. Non-inflated Type I error rates are important since it is vital to retain valid items since item development is often expensive. In addition, adequate power to identify misfitting items is also important since IRT applications rely on the model fitting the data. Graphical displays, although they suffer from some degree of subjective interpretation, can often provide meaningful insights into the nature of model-data misfit and assist in interpreting the meaningfulness of an effect from a statistical significance test.

The newly-proposed nonparametric approach, RISE, overcomes the main problems of traditional fit statistics such as arbitrariness of grouping and inaccuracy of 
the null distribution assumption. Essentially, the idea of RISE is based on the weak assumption proposed by Douglas and Cohen (2001) that for moderate sample sizes, nonparametrically-derived ICC will be close to the parametric ICC. The significance level of RISE is determined by the established parametric bootstrapping procedure which forms an empirical distribution of the difference between the nonparametric ICC and parametric ICC. Beyond its statistical properties mentioned above, an additional advantage of the nonparametric approach is the convenient graphical representation of model misfit. This feature can provide the researcher or practitioner with visual guidance about the location and type of misfit. On the other hand, in the aspect of examining model misfit consequences, both the test score distribution and the TCC were used to address this issue. Basically, misfitting items detected by three fit statistics were replaced by fitting items to see the change of score distribution or TCC. If the change was not significant for classification, the conclusion was that there was no practical consequence for the given data and condition.

In the dissertation, Study 1 was a simulation study to examine the statistical properties of RISE thoroughly and comprehensively. Ability distribution, sample size, test length and model were four factors considered as they may influence performance of fit. Among the thirty-six conditions from the four factors, RISE outperformed $G^{2}$ and $S-X^{2}$ in that it controlled Type I error rates and provided adequate power. However, $G^{2}$ exhibited different degrees of inflated Type I error rates on all conditions while less power than RISE.S-X did not control Type I error rates for the conditions involved in skewed ability distributions except the 500-examinee and 60-item condition, which may be due to the requirement of distribution assumption of $S-X^{2}$ and thus the limited use of the software to compute $S-X^{2}$. However, it did exhibit controlled Type I error rates for the conditions under normal ability distributions although the power was much 
lower than RISE especially for small and medium sample sizes. In the simulation study, model misfit consequences on the score distribution and TCC were also examined. The score distribution and TCC did not change significantly before and after replacement of the misfitting items. This result is probably due to the small amount of misfit simulated at the test score level.

In Study 2, the three fit statistics were applied to empirical data for three sample sizes. The number of misfitting items detected increased by sample size among all three fit statistics. RISE and $S$ - $X^{2}$ detected comparable number of misfitting items while $G^{2}$ detected a lot (e.g., at sample size 10,000, $G^{2}$ detected $95 \%$ items as misfitting items).

Graphical displays were implemented in this study to help judge the accuracy of detection. One advantage of the nonparametric approach is that the difference between the nonparametric and parametric ICCs provides evidence regarding the location and magnitude of misfit of each detected item. One phenomenon for the parametric approach, especially $G^{2}$, was that the reasonably good items shown by the residual plots were still detected by the fit statistic as misfitting items. Model misfit consequence on score distributions with small, medium and large sample sizes and real data were also investigated. The conclusion was the same as the simulation study. However, the results demonstrated the capability of RISE in the accuracy of misfit item detection.

Although the results in the dissertation provided strong evidence that the proposed $R I S E$ is a convincing fit statistic for the studied conditions, further exploration is still necessary to understand the nonparametric approach in depth. For instance, other smoothing techniques to produce the nonparametric ICC can be implemented to compare with kernel smoothed ICCs. This may influence the fitting ICC and thus the whole RISE procedure. It may also be worthwhile to examine the statistical properties for other models. In the current study, only four models, 2PLM, 3PLM, GRM and 
GPCM were applied. In future research, the 1PLM/PCM combination may be of interest since it is also commonly used in some testing programs (e.g., state achievement testing and the health field). Furthermore, it may be useful to explore the performance between a variety of parameter estimation methods in obtaining the optimal item parameter values as the robustness of item parameter estimation techniques is desired in the nonparametric approach. Other than parameter estimation techniques, the number of evaluation points may also influence the parameter estimation procedure, so future research will include this factor. In addition, model misfit consequences on score distributions can be further assessed by simulating large amount of misfit which is expected to make a difference. Also, other aspects of misfit consequence on the invariance property and parameter estimates can be conducted. Finally, it would be worthwhile to compare RISE with newly emerging fit statistics (e.g., Bayesian approach) as according to the research literature, Beyesian approach has also exhibited good performance (Sinharay, 2005, Stone, 2000a ).

While it is unreasonable to expect IRT models to provide perfect fit to the test data, the evidence is substantial that they provide an excellent framework for solving measurement problems. Model-data fit is a systematic and comprehensive procedure (see Hambleton \& Han, 2005). Although it is clear from this study that the nonparametric approach is promising to identify misfitting items and provide the graphical representation of misfit, more and more problems may still be ahead and have not been solved. For example, as IRT has been widely accepted and applied in variety of fields, more and more practical problems such as how to measure and understand model misfit consequence are challenging. All in all, the new method is expected to bring a new direction for improving the utility of IRT modeling for measurement problem-solving in educational and psychological testing. 


\section{BIBLIOGRAPHY}

Ankenmann, R. (1994). Goodness of fit and ability estimation in the graded response model. Unpublished manuscript.

Bjorner, J.B., Smith, K.J., Stone, C, \& Sun, X.W. (2007). IRTFIT: A macro for item fit and local dependence tests under IRT models [Computer program]. QualityMetric, Inc.

Bock, R. D. (1972). Estimating item parameters and latent ability when responses are scored in two or more nominal categories. Psychometrika, 37, 29-51.

Bolt, D. M. (2002). A Monte Carlo comparison of parametric and nonparametric polytomous DIF detection methods. Applied Measurement in Education, 15, 113-141.

Chon, K. H., Lee, W., \& Ansley, T. N. (2007). Assessing IRT model-data fit for mixed format tests (Research Report No. 26). Iowa City, Iowa: Center for Advanced Studies in Measurement and Assessment, the University of Iowa. Retrieved from http://education.uiowa.edu/casma

Cohen, J. (1994) The effect of climate perturbations on extratropical cyclogenesis along the east coast of north America. Doctoral dissertation, Columbia University, NY.

DeMars, C.E. (2005). Type I error rate for PARSCALE's fit index. Educational and Psychological Measurement, 65(1), 42-50.

Donoghue, J. R., \& Hombo, C. M. (1999, June). Some asymptotic results on the distribution of an IRT measure of item fit. Paper presented at the meeting of the Psychometric Society, Lawrence, KS.

Donoghue, J. R., \& Hombo, C. M. (2001, April). The distribution of an item-fit measure for polytomous items. Paper presented at the meeting of the National Council of Measurement in Education, Seattle, WA.

Douglas, J. (1997). Joint consistency of nonparametric item characteristic curve and ability estimation. Psychometrika, 62, 7-28.

Douglas, J. \& Cohen, A. S. (2001). Nonparametric item response function estimation for assessing parametric model fit. Applied Psychological Measurement, 25, 234-243.

Embretson, S. E., \& Reise, S.P. (2000). Item response theory for psychologists. Mahwah, NJ: Erlbaum.

Glas, C. A. W. (1998). Detection of differential item functioning using Lagrange multiplier tests. Statistica Sinica, 8(1), 647-667. 
Glas, C. A. W., \& Suárez Falcón, J. C. (2003). A comparison of item-fit statistics for the three parameter logistic model. Applied Psychological Measurement, 27, 87-106.

Guttman, I. (1967). The use of the concept of a future observation in goodness-of-fit problems. Journal of the Royal Statistical Society B, 29, 83-100.

Hambleton, R. K. (1989). Principles and selected applications of item response theory. In R. L. Linn (Eds.), Educational measurement (3rd ed., pp. 143-200). New York: Macmillan.

Hambleton, R. K., \& Han, N. (2004). Assessing the fit of IRT models: Some approaches and graphical displays. Paper presented at the meeting of the National Council on Measurement in Education, San Diego, CA.

Hambleton, R. K., \& Han, N. (2005). Assessing the fit of IRT models to educational and psychological test data: A five step plan and several graphical displays. In W. R. Lenderking \& D. Revicki (Eds.), Advances in health outcomes research methods, measurement, statistical analysis, and clinical applications (pp. 57-78). Washington: Degnon Associates.

Hambleton, R. K., \& Swaminathan, H. (1985). Item response theory: Principles and applications. Boston, MA: Kluwer-Nijhoff.

Hambleton, R.K., Swaminathan, H., \& Rogers, H.J. (1991). Fundamentals of item response theory. Newbury Park, CA: Sage.

Kang, T., \& Chen, T.T. (2008). Performance of the generalized S-X2 item fit index for polytomous IRT models. Journal of Educational Measurement, 45(4), 391-406.

Kingston, N., \& Dorans, N. (1985). The analysis of item-ability regressions: an exploratory IRT model fit tool. Applied Psychological Measurement, 9, 281-288.

Li, S., \& Wells, C. S. (2006). A model fit statistic for Samejima's graded response model. Paper presented at the meeting of National Council on Measurement in Education, San Francisco, CF.

Liang, T., Han, K.T., \& Hambleton, R. K. (2009). ResidPlots-2: Computer software for IRT graphical residual analyses [computer program]. Applied Psychological Measurement, 33(5), 411-412.

Liang,T., \& Wells, C.S. (2009). A model fit statistic for generalized partial credit model. Educational and Psychological Measurement, 69(6), 913-928.

Liang, T., \& Wells, C. S. (2008). A nonparametric approach for assessing model fit in a mixed format test. Paper presented at the meeting of the National Council on Measurement in Education, NY.

Lord, F. M., \& Wingersky, M. S. (1984). Comparison of IRT true-score and equipercentile observed-score "equatings." Applied Psychological Measurement, 8, 453-461. 
Masters, G. N. (1982). A Rasch model for partial credit scoring. Psychometrika,47, 149-174.

McKinley, R. \& Mills, C. (1985). A comparison of several goodness-of-fit statistics. Applied Psychological Measurement, 9, 49-57.

Muraki, E. (1992). A generalized partial credit model: Application of an EM algorithm. Applied Psychological Measurement, 16, 159-176.

Muraki, E., \& Bock, R. D. (2003). PARSCALE 4: IRT item analysis and test scoring for rating-scale data [computer program]. Chicago, IL: Scientific Software.

Orlando, M., \& Thissen, D. (2000). Likelihood-based item-fit indices for dichotomous item response theory models. Applied Psychological Measurement, 24, 50-64.

Orlando, M., \& Thissen, D. (2003). Further investigation of the performance of S-X2: An item fit index for use with dichotomous item response theory models. Applied Psychological Measurement, 27, 289.

Press, W., Teukolsky, S., Vetterling, \& Flannery, B. (1992). Numerical recipes in Fortran 77. ( $2^{\text {nd }}$ ed.).Cambridge University Press.

Ramsay,J.O. (1991). Kernel smoothing approaches to nonparametric item characteristic curve estimation. Psychometrika, 60, 323-339.

Reise, S. P. (1990). A comparison of item- and person-fit methods of assessing model data fit in IRT. Applied Psychological Measurement, 14, 127-137.

Rogers, H., \& Hattie, J. (1987). A Monte Carlo investigation of several person and item fit statistics for item response models. Applied Psychological Measurement, 11, 47-57.

Samejima, F. (1969). Estimation of latent ability using a response pattern of graded scores (Psychometric Monograph No. 17). Iowa City, IA: Psychometric Society.

Sinharay, S. (2005). Assessing fit of unidimensional item response theory models using a Bayesian approach. Journal of Educational Measurement, 42, 375-394.

Stone, C. A. (2000a). A Monte Carlo based null distribution for an alternative fit statistic. Journal of Educational Measurement, 37, 58-75.

Stone, C.A , \& Hansen, M.A. (2000). The effect of errors in estimating ability on goodness-of-fit tests for IRT models. Educational and Psychological Measurement, 60(6), 974-991.

Stone, C. A. \& Zhang, B. (2003). Assessing goodness of fit in item response theory models: A comparison of traditional and alternative procedures. Journal of Educational Measurement, 40, 331-352. 
Van der Linden, W.J. \& Hambleton, R.K. (1997).Handbook of modern item response theory. New York: Springer-Verlag.

Von Schrader, S., Ansley, T.N., \& Kim, S. (2004, April). Examination of item fit indices for polytomous item response models. Paper presented at the meeting of the National Council on Measurement in Education annual meeting, San Diego, CA.

Wainer, H., \& Mislevy, R. J. (1990). Item response theory, itemcalibration, and proficiency estimation. In H. Wainer, N.J.Dorans, R.Flaugher, B.F.Green, R.J. Mislevy, L. Steinberg, \& D. Thissen, Computerized adaptive testing: A primer (pp. 65-101). Hillsdale NJ: Erlbaum.

Wang, T., Kolen, M. J., \& Harris, D. J. (2000). Psychometric properties of scale scores and performance levels for performance assessment using polytomous IRT. Journal of Educational Measurement, 37, 141-162.

Wells, C. S. \& Bolt, D. M. (2008). Investigation of a nonparametric procedure for assessing goodness-of-fit in item response theory. Applied Measurement in Education.

Wright, B., \& Mead, R. (1977). BICAL: Calibrating items and scales with the Rasch model (Research Memorandum No. 23). Chicago: University of Chicago, Department of Education, Statistical Laboratory.

Yen, W. (1981). Using simulation results to choose a latent trait model. Applied Psychological Measurement, 5, 245-262.

Zimowski, M. F., Muraki, E., Mislevy, R. J., \& Bock, R. D. (2003). BILOG-MG 3 [computer program]. Chicago, IL: Scientific Software. 